\title{
THE STATE Of NePAL BIRDS 2010
}

\section{Carol Inskipp ${ }^{1}$, Hem Sagar Baral ${ }^{2}$, Tim Inskipp $^{3}$ \& Alison Stattersfield ${ }^{4}$}

${ }_{1,3}$ Herneside, Welney, Wisbech, Cambs PE14 9SB, UK

${ }^{2}$ PO Box 10918, Himalayan Nature, Lazimpat, Kathmandu, Nepal

${ }^{4}$ BirdLife International, Wellbrook Court, Girton Road, Cambridge CB3ONA, UK.

1'inskipp@btinternet.com (corresponding author), ${ }^{2}$ hem.baral@gmail.com, ${ }^{3}$ inskipp@ @btinternet.com, ${ }^{4}$ Ali.Stattersfield@birdlife.org

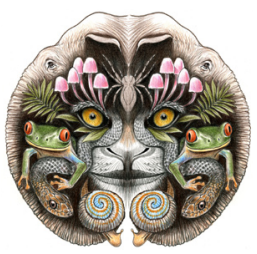

ISSN

Online 0974-7907

Print 0974-7893

Abstract: The national status of Nepal's birds was determined using the IUCN Red List criteria and following IUCN's regional guidelines. Records of all species identified as potentially at high risk were extracted from all relevant references in a comprehensive, up-to-date Nepal bird bibliography. In addition, numerous previously undocumented records were obtained from observers in Nepal. The initial list of potentially threatened species was revised as records were accumulated. Literature reviews were made of current pressures on Nepal's birds, responses to these pressures and recommendations for the future. Finally a comprehensive summary table was compiled for all nationally threatened species, including their world distribution, global threat status, national threat status, occurrence, habitat, main altitudinal range, population, key threats, research needs, and key conservation interventions needed. In 2010, 149 bird species (17\% of the total recorded) of Nepal's birds were considered nationally threatened: 53 Critically Endangered species, 48 Endangered and 47 Vulnerable. Near Threatened species were not assessed due to lack of time available for the necessary research. An additional 16 species were considered threatened in 2010 compared with 2004; no species assessed as threatened in 2004 was considered non-threatened in 2010. When habitat types are considered wetland species are the most threatened (35\% of the total wetland species), followed by grassland species $(23 \%)$. When altitudinal preferences are considered lowland species are the most threatened $(36 \%$ of all lowland species), followed by species only occurring in the middle hills (17\%). Human activities leading to habitat loss and damage are the major threats, with agriculture the root cause; hunting and trapping are other important threats. Effective responses for conservation include Nepal's protected areas network, community forestry, designation of Ramsar sites, National Wetland Policy implementation, surveys of globally threatened species and their conservation needs, and conservation awareness activities. Recommendations for future actions by non governmental organisations are made.

Keywords: Assessment, conservation, nationally threatened birds, Nepal, pressures, response, status.

Nepali Abstract: नेपालमा पाईने चराहरूको अवस्था तयार गर्नको लागि आइयूसीएन् को संकटमा परेका जीवहरूको सूची तयार गर्दा प्रयोग गरिने आधार र निर्देशिकाको पालना गरियो। संकटमा पर्न लागेका सम्भावित चराहरूको सची तयार गरि तिनको स्थिति अद्यावधि गर्नका लागि नेपालका चराहरूबारेको परिमार्जित सन्दर्भसचीबाट आवश्यक सन्दर्भसामाग्रीहरू छानेर जानकारी लिइयो । यसका साथै विभिन्न चरा अवलोकनकर्ताहरूबाट पहिले थाहा नपाइएका अभिलेखहरू पनि प्राप्त गरियो। यसरी अभिलेखहरूको ओइरो लागदै गए पछि, शुरुमा प्रयोग गरिएको संकटमा परेका संभावित चराहरूको सचीमा थप संसोधन गरियो। नेपालका चराहरूलाई पर्न गएका विद्यमान खतराहरू, संरक्षणका प्रयाशहरू र भविष्यलाई लक्षित गरि राखेका सुकावहरूका संकलित सन्दर्भ-सामाग्रीको विस्तृत अध्ययन गरियो। अन्त्यमा गहन अध्ययनपश्चात राष्ट्रियस्तरमा संकटमा रहेका प्रजातिको विश्वव्यापी भौगोलिक वितरण, विश्वव्यापी खतराको स्थिति, राष्ट्रियस्तरमा खतराको स्थिति, नेपालमा रहंदाको रैथाने/आगन्तुक अवस्था, प्रमुख बासस्थान, प्रजाति विशेषले मनपराउने मुख्य उचाईंको रेन्ज, अनुमानित संख्या, प्रमुख खतराहरू, अनुसन्धानका लागि प्राथमिकताहरू र संरक्षणकालागि प्रमुख उपायहरूसहितको सारांश-टेबुल तयार पारियो। सन् २०१० मा, नेपालका १४९ चराका प्रजातिहरू (कुल संख्याको १७\%) राष्ट्रयस्तरमा संकटमा रहेको पाइयो, जसमध्ये प३ प्रजाति अतिसंकटापन्न, ૪६ संकटापन्न र ४७ संवेदनशील अवस्थामा वर्गीकरण गरियो। संकटनजिक रहेका थप प्रजातिहरू आवश्यक अनुसन्धान गर्न समय अपुग भएकाले यिनलाई खासै मूल्यांकन गरिएन। सन २००४ मा संकटमा रहेका सबै चराहरू यसपाली पनि सचीकत भए साथै थप १६ प्रजातिहरू संकटमा रहेका चराहरूको सचीमा परे। बासस्थानका हिसाबले हेर्दा सिमसारमा आश्रित चराहरू सबभन्दा बढि संकटमा परेका (सिमसारमा आश्रित चराको ३५\%) र त्यसपछि, घाँसेमैदानमा आश्रित चराहरू (२३\%) परेको पाइयो। उचाईंका हिसाबले विभिन्न भेकका चरामा, तराईमा पाइने चराहरू (तराईका सम्पर्ण चराको ३६\%) सबैभन्दा बढि त्यसपछि पहाडमामात्र पाइने चराहरू (१७\%) संकटमा रहेको पाइयो। कृषिका लागि गरिने मानिसजन्य क्रियाकलापहरूले गर्दा बासस्थानको ह्रास-बिनाश हुनु चराका लागि प्रमुख खतरा हो भने चोरी-शिकारी तथा चरालाई पुग्ने अन्य बाधा-व्यवधान पनि महत्वपूर्ण खतरा मानिन्छन्। संरक्षणलाई प्रभावकारी राख्नका लागि नेपालमा संरक्षित क्षेत्रहरूको सः्जाल, सामुदायिक वन, रामसार क्षेत्रहरू, राष्ट्रिय सिमसार नीतिको कार्यान्वयन, नेपालमा पाइने विश्वमै संकटमा परेका चराहरूको सर्वेक्षण तथा संरक्षणका प्रयाशहरू, संरक्षणका लागि चेतनामुलक कार्यकमहरू संचालित छन् । गैर-सरकारी संस्थाहरूले भविष्यमा गर्नुपर्ने संरक्षणका लागि कार्यनीतिहरूको सुकाव प्रस्तुत गरिएको छ।

DOI: http://dx.doi.org/10.11609/JoTT.03276.933 | ZooBank: urn:Isid:zoobank.org:pub:A230A337-E2D3-41C3-A1FE-32AA6FADFE03

Editor: J.W. Duckworth, IUCN SSC, Bath, UK.

Date of publication: 26 January 2013 (online \& print)

Manuscript details: Ms \# 03276 | Received 30 July 2012 | Final received 04 December 2012 | Finally accepted 08 December 2012

Citation: Inskipp, C., H.S. Baral, T. Inskipp \& A. Stattersfield (2013). The state of Nepal birds 2010. Journal of Threatened Taxa 5(1): 3473-3503; doi:10.11609/ JoTT.03276.933

Copyright: (c) Inskipp et al. 2013. Creative Commons Attribution 3.0 Unported License. JoTT allows unrestricted use of this article in any medium for non-profit purposes, reproduction and distribution by providing adequate credit to the authors and the source of publication.

Funding: Jensen Foundation, Darwin Initiative and BirdLife International.

\section{Competing Interest: None.}

Acknowledgements: We are very grateful to the Jensen Foundation, Darwin Initiative and BirdLife International for generously supporting this study. Special thanks go to Hum Gurung, Chief Executive Officer, Bird Conservation Nepal (BCN) who managed the State of Nepal Birds 2010 project and to Ishana Thapa, Jyotendra Thakuri, Menuka Basnyat, Sushma Shrestha, Anand Chaudhary and Mitra Pandey from BCN and Tris Allinson, Jenny Birch and Mike Crosby from BirdLife International for their contributions. Compiling the data would not have been possible without the generous donation of unpublished records by numerous observers and many other people provided useful comments on the draft results. We warmly thank the reviewers of the manuscript, Dr Rajan Amin, Dr Will Duckworth and Laxman Poudyal for their very useful comments. The results of this study are also presented in a more popular form in a report published in Nepal by BCN and the Department of National Parks and Wildlife Conservation, and is available from the BirdLife International website www.birdlife.org. A complete list of names of all those who contributed records or who made comments is given in this report. 


\section{INTRODUCTION}

Nepal is renowned internationally for its rich diversity of bird species. Although the country possesses an area of just $147,181 \mathrm{~km}^{2}(0.1 \%$ of the world's total landmass), it accounts for about $8 \%$ of the world's bird species, with 867 species recorded up to the end of 2010, including about 800 species that are regularly recorded (Bird Conservation Nepal 2011, unpubl. data). In terms of globally threatened bird species, 36 have been recorded in Nepal based on the categorisation in BirdLife International (2010), 25 of which occur regularly and are included in this study.

A total of 27 Important Bird Areas (IBAs) has been identified in Nepal covering forests (22 IBAs), grasslands (four) and freshwater ecosystems (10); some IBAs contain more than one of these ecosystems (Baral \& Inskipp 2005). Important Bird Areas are key sites for bird conservation which have been identified using standardised global criteria (BirdLife International 2011). In Nepal 24 IBAs support globally threatened species, 13 have restricted-range species, 24 have biome-restricted species and eight qualify as IBAs because they hold large congregations of waterbirds (Baral \& Inskipp 2005).

The first assessment of the threat status of Nepal's birds: Threatened Birds of Nepal was published in 1996 (Baral et al. 1996). This report was succeeded by The State of Nepal's Birds 2004 (Baral \& Inskipp 2004) eight years later. Better documentation on species' status enabled the latter report to be much more detailed than the 1996 report. It included an account of the threats to Nepal's birds, an assessment of national threat status for individual species considered to qualify at national level for IUCN Categories Vulnerable, Endangered and Critically Endangered categories and a comprehensive review of all Endangered and Critically Endangered species.

This paper presents an assessment of national threatened species conducted in 2010, a review of current pressures on Nepal's birds and responses already taken, and some recommendations for actions by NGOs to address the status of Nepal's nationally threatened birds. A more detailed account of this assessment is given by Bird Conservation Nepal (BCN) and Department of National Parks and Wildlife Conservation (DNPWC) (2011).

\section{METHODS}

The national status of Nepal's birds was determined using the IUCN Red List criteria, and following IUCN's regional guidelines in their application. This involved considering a few issues that are not encountered at the global level, for example species that do not reproduce in the country, but are still dependent upon its resources for their survival (IUCN 2003). A number of species on the national threatened list falls into this category, for example Black Stork Ciconia nigra, Pied Harrier Circus melanoleucos, Greater Spotted Eagle Aquila clanga, Baillon's Crake Porzana pusilla, White-throated Bushchat Saxicola insignis and Yellow-breasted Bunting Emberiza aureola. It is possible to consider species that are nonnative but have been introduced to the country (IUCN 2003), but no species occurring in Nepal fall into this category.

The status of Nepal's birds in 2010 was assessed by first undertaking a literature search to update a comprehensive bibliography of Nepal's birds (Inskipp \& Inskipp 2010). An initial list of potentially high risk species was drawn up. Records of these species, including number of individuals, date and location were extracted from all relevant references in the bibliography and compiled on species forms. Numerous previously undocumented and valuable records were obtained from observers in Nepal, some of whom completed species records forms. The forms provided details of the location and dates of records, and the numbers of each species recorded, as well as any information that was available on threats. Records from observers outside Nepal had already been acquired from reports or were located by research online. As records accumulated some species were found to be better off than expected and were dropped from the initial list of potentially threatened species. A few species were added to the initial list as other observers alerted us to their rarity.

Only resident species and summer and winter visitors were assessed (755 species in total), not passage migrants (29 species), vagrants (74 species), nor extinct or extirpated species (nine species).

Literature reviews were made of current pressures on Nepal's birds and also responses to these pressures that have already been made, as well as those that are recommended for the future by NGOs.

A comprehensive summary table was compiled for

Abbreviations: BCN - Bird Conservation Nepal; CBD - Convention on Biological Diversity; DNPWC - Department of National Parks and Wildlife Conservation; IBA - Important Bird Area; IUCN - International Union for Conservation of Nature; NBS - National Biodiversity Strategy; NGO - Non Governmental Organisation; 


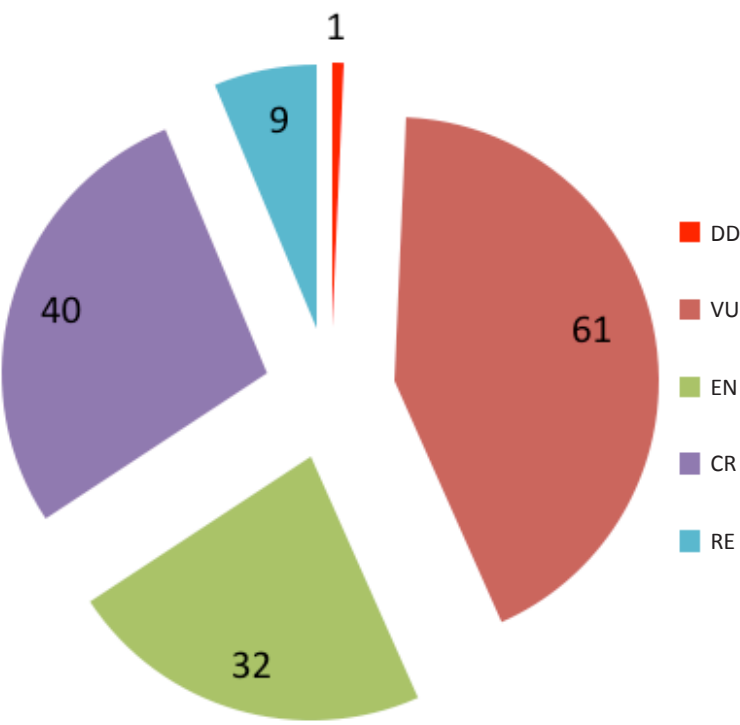

Figure 1. Threatened bird species assessments in 2004 Source: Baral \& Inskipp (2004)

all nationally threatened species, including their world distribution, global threat status, national threat status, occurrence, habitat, main altitudinal range, population, key threats, research needs, and key conservation interventions needed (see results).

\section{RESULTS}

\section{State of Nepal's birds}

Comparison of results in 2004 and 2010: The 2010 assessment of the status of Nepal's birds showed that an alarming 149 bird species (17\%) are threatened at the national level (Table 1). As many as 99 species are thought to be Critically Endangered or Endangered, meaning there is an extremely high or very high risk of their extirpation in Nepal in the near future.

An additional 16 species are considered nationally threatened compared with the 2004 assessment of 133 threatened species (Baral \& Inskipp 2004) (Fig. 1). No species assessed as threatened in 2004 was considered non-threatened in 2010 . There is a much higher number of Critically Endangered species (61) in 2010 than in 2004 (40 species) and also a higher number of Endangered species (38) compared with the 2004 total of 32 species. The number of species categorised as Vulnerable was higher in 2004 (61) than in 2010 (50) (Fig. 2). There was also one Data Deficient species in 2004 (Baral \& Inskipp 2004) but none in 2010. Near Threatened species were not assessed due to lack of time available for the necessary research.

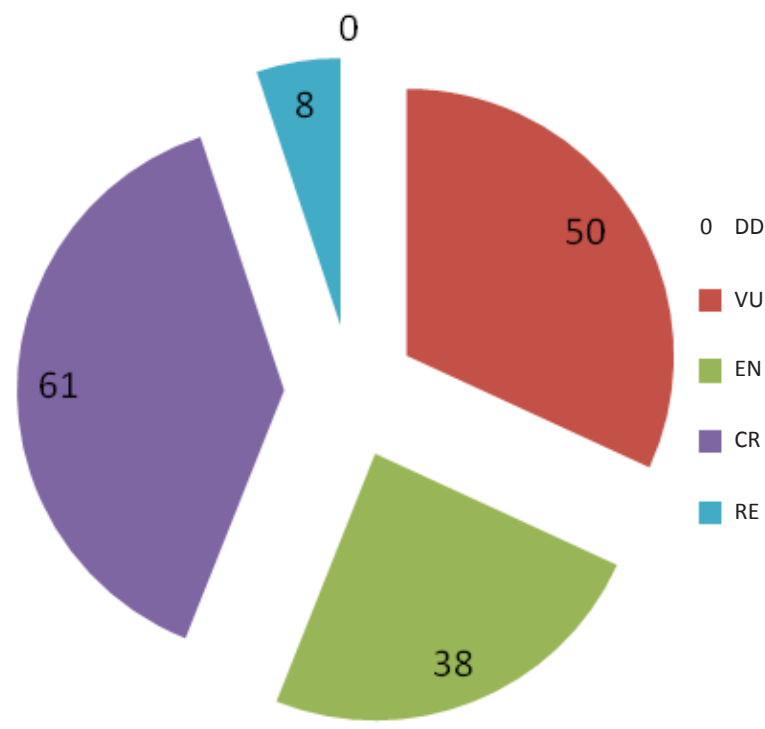

Figure 2. Threatened bird species assessments in $\mathbf{2 0 1 0}$

Habitat preferences of nationally threatened birds: The 2010 study found that over half (53\%, 79 species) of Nepal's nationally threatened birds inhabit forests. Nationally threatened species inhabiting wetlands total 40 (27\%), grasslands 23 (15\%), cultivation 12 (8\%), scrub seven $(5 \%)$, open country $14(9 \%)$, near human habitation four (3\%), and semi-desert one (1\%).

When all species regularly occurring in Nepal are considered, 474 species (59\%) inhabit forests, 184 (23\%) cultivation, 115 (14\%) wetlands, 100 (13\%) grasslands, 101 (13\% scrub), 75 (9\%) open country, 30 (4\%) near human habitation and $19(2 \%)$ in semi-desert.

This means that wetland species are the most threatened (35\% of the total of wetland species), followed by species of grasslands (23\%), open country $(18 \%)$, forests $(17 \%)$, near human habitation $(13 \%)$, in cultivation (7\%), scrub ( 7\%), and semi-desert (5\%) (Fig. 3)

Altitudinal preferences of nationally threatened birds: This study showed that over half ( $56 \%, 83$ species) of Nepal's nationally threatened species are only found in the lowlands (75-1000 m). Some 19\% (28 species) occur in the lowlands as well as in the middle hills (75-3050 m), 13\% (20 species) only in the middle hills (1000-3050 m), just 5\% (8 species) in the middle hills and higher altitudes (above $3050 \mathrm{~m}$ ) and 1\% (2 species) only at higher altitudes (above 3050m).

When all species regularly occurring in Nepal are considered, 233 species (29\%) are only found in the lowlands (75-1000 m). A total of 250 species (31\%) occur in the lowlands as well as the middle hills $(75-3050 \mathrm{~m})$, 115 species (14\%) only in the middle hills (1000-3050 


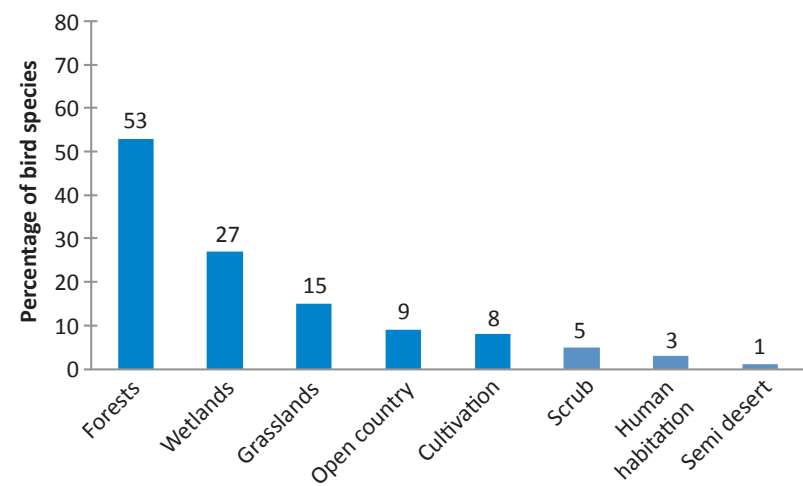

Figure 3. Proportion of nationally threatened birds occurring in different habitat types

N.B. Figures are not cumulative because several species are found in more than one habitat type. For instance, Grey-headed Fish Eagle Ichthyophaga ichthyaetus and Lesser Fish Eagle I. humilis require both forests and wetlands as they breed in forests close to rivers or lakes.

m), 181 species (23\%) in the middle hills and higher altitudes (above $3050 \mathrm{~m}$ ), and 21 species (3\%) only at higher altitudes (above 3050m).

This means that lowland species are the most threatened (36\% of all lowland species), followed by species only occurring in the middle hills $(17 \%), 11 \%$ of species in the lowlands as well as the middle hills, $5 \%$ of species only at high altitudes, and $4 \%$ of species in the middle hills and higher latitudes (Fig. 4).

Globally threatened species: All the globally threatened species regularly occurring in Nepal met the criteria for being nationally threatened.

\section{Pressures on Nepal's birds}

Effects of habitat losses and damage: Human activities are putting enormous pressure on Nepal's bird populations. The 2010 study revealed that those leading to habitat loss and damage were the major threat affecting a total of $128(86 \%)$ of the species of birds at risk nationally, at least to some degree.

Impacts of agriculture: A recent study of the impacts of agriculture on Nepal's birds concluded that the spread of agriculture and changes in agricultural practices are the major root causes of loss and damage to natural habitats (Inskipp \& Baral 2011).

Conversion for agriculture is the chief cause of deforestation, while over-grazing by livestock and overharvesting for fodder are the main causes of forest degradation (South Asia Co-operative Environment Programme 2010). Overgrazing presents major problems to lowland reserves, which protect almost all of Nepal's remaining lowland grasslands (Baral 2001). Furthermore, the level of livestock grazing is considered one of the most serious threats to the ecological

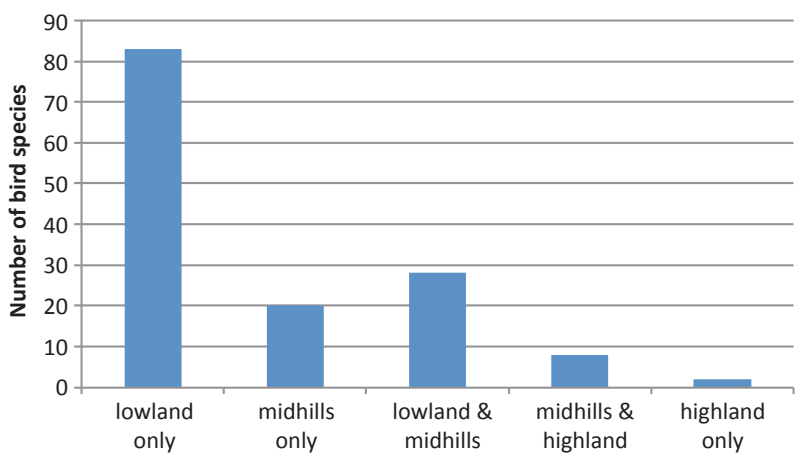

Figure 4 Numbers of nationally threatened birds occurring at different altitudes

N.B. The total number of threatened species plotted in the bar chart is rather less than the total number of species threatened as a few species fall outside the categories plotted.

integrity of the mid-hill and highland protected areas (HMGN/MFSC 2002). Livestock overgrazing is said to be responsible for damage to wetlands (IUCN 2004) and the low biodiversity of upland grasslands (MoEST 2006). In addition, heavy grazing and browsing promote the spread of an invasive alien creeper, Bittervine Mikania micrantha, which is now a particularly dangerous threat in Nepal (Siwakoti 2007).

(a) Pesticides: Pesticides are widely and increasingly used in agriculture, especially in the lowlands (Atreya 2008) and could be a significant threat to many species (Inskipp \& Baral 2011).

Proof of pesticide impacts on birds, which can only be determined by analysis of eggs or bodies, has not been carried out on Nepal's birds till date. In this study pesticide poisoning is considered a possible threat to 21 nationally threatened species, mainly birds of prey and large wading birds; however, many more species could be affected.

(b) Fertilisers: Trend analysis and the opinion of traders show that fertiliser use has increased by about $11.5 \mathrm{~kg}$ per ha every year in recent years (Thapa 2006). Widespread contamination of agricultural run-off by nitrogen and phosphate nutrients from fertilisers has led to eutrophication of lowland wetlands. Eutrophication has eventually resulted in low oxygen levels in water and the death of invertebrate and fish food supplies for birds (Kafle et al. 2007, 2008).

(c) Poisoning by diclofenac: Poisoning by diclofenac, a drug used for livestock ailments has led to drastic declines in vulture populations in the Indian subcontinent, including Nepal (Oaks et al. 2004; Shultz et al. 2004). For example, White-rumped Vulture Gyps bengalensis, formerly the most common Nepal vulture up to $1000 \mathrm{~m}$ (Inskipp \& Inskipp 1991), and the once fairly common 
and widespread Slender-billed Vulture G. tenuirostris have declined to such a level and so sharply that both species are now categorised as nationally Critically Endangered.

\section{Pressures on forest birds}

The high proportion of forest bird species nationally at risk can be partly attributed to forest depletion being one of the major environmental issues in the country (South Asia Co-operative Environment Programme 2010) and also because forests comprise the major natural habitat in Nepal.

According to the 2005 FAO Global Forest Resources Assessment, 25\% of Nepal was covered in forest in 2005. This forest includes plantations, but these form a very small proportion of forest cover in Nepal $(53,000 \mathrm{ha})$. The total loss of forest area between 1990 and 2005 was $25 \%$. During the same period degraded forest (wooded areas with tree canopy 5-10\%), increased from 1,180,000-1,897,000 ha (Forestry Nepal 2005).

Conversion of land to agriculture is the major cause of deforestation in Nepal. Other causes of deforestation are the growth of settlements and infrastructure, illicit tree-felling and the transboundary timber trade (South Asia Co-operative Environment Programme 2010).

The majority of Nepalis depend on the country's forests for their essential requirements for fuel, livestock fodder and other basic materials.

The 2010 assessment found that most threatened forest birds inhabit the tropical and subtropical and lower temperate zones where forests have been most depleted; for instance Yellow-vented Warbler Phylloscopus cantator, Abbott's Babbler Malacocincla abbotti and White-naped Yuhina Yuhina bakeri. Many of the threatened forest birds require dense, moist conditions, a well-developed understorey or epiphytic growth, for example Broad-billed Warbler Tickellia hodgsoni, Rufous-throated Wren Babbler Spelaeornis caudatus and Himalayan Cutia Cutia nipalensis.

A total of 14 forest species from these zones have not been recorded for at least ten years: Pale-headed Woodpecker Gecinulus grantia, Blyth's Kingfisher Alcedo hercules, Mountain Imperial Pigeon Ducula badia, Asian Fairy Bluebird Irena puella, Yellow-cheeked Tit Parus spilonotus, Rufous-faced Warbler Abroscopus albogularis, Coral-billed Scimitar Babbler Pomatorhinus ferruginosus, Rufous-throated Wren Babbler Spelaeornis caudatus, Spotted Wren Babbler Spelaeornis formosus, Silver-eared Mesia Leiothrix argentauris, Rufous-backed Sibia Heterophasia annectans, White-hooded Babbler Gampsorhynchus rufulus, Scarlet-backed Flowerpecker
Dicaeum cruentatum and Yellow-vented Flowerpecker D. chrysorrheum.

A high proportion (47\%; 37 species) of threatened forest birds was found to inhabit broadleaved evergreen forests, an especially threatened habitat, and $32 \%$ of forest birds (25 species) (for example Ruddy Kingfisher Halcyon coromanda), are largely confined to evergreen forests in the tropical and/or subtropical zones, where these forests are of very limited extent.

Suitable habitat for some threatened forest species may still be unexplored, especially in upper temperate and subalpine forests and so these species may be less threatened than currently believed.

Besides habitat loss, some forest species, especially Galliformes and owls, suffer from hunting and trapping.

\section{Loss of invaluable habitat linkages}

Despite losses and degradation, there is still forest cover on steep slopes, and this is likely to be untouched if access remains difficult; thus providing protection for birds and potential reservoirs of bird populations for recolonisation. However, the continuation of forest cover on steep slopes will not help to protect species which winter in the lowlands if forest cover in the lowlands is cleared.

The review of pressures on birds found that forest losses have been so widespread and extensive in the lower and middle hills that invaluable habitat linkages between forests in the high Himal and lowlands or lower hills have been lost. As a result many bird species no longer have available the continuum of habitats that they require to move altitudinally with the seasons and their distributional range is therefore restricted e.g. Barred Cuckoo Dove Macropygia unchall, Dark-sided Thrush Zoothera marginata, and Lesser Shortwing Brachypteryx leucophrys.

Furthermore, the loss of a continuum of habitats across a high altitudinal gradient means that many species are much less able to shift their distribution according to climate change and are therefore more at risk.

\section{Pressures on wetland birds}

Wetland birds were found to be the most threatened in Nepal (35\% of wetland species are nationally threatened). Threats have significantly increased since 2004. Widespread threats include drainage for agriculture, unsustainable harvesting of resources, diversion and abstraction of water for farmland irrigation, overgrazing of shorelines and marshes, and mining of gravel from river beds. Many species are suffering from 
water pollution, hunting, trapping, disturbance and destruction of feeding and nesting sites. Water pollution from agricultural chemicals has been identified as a particularly serious threat to lowland wetlands (Kafle et al. 2007, 2008).

Overfishing and fish-poisoning: Overfishing and fishpoisoning are major threats to large fish-eating birds. All these species have declined in Nepal's wetlands almost certainly because overfishing has significantly reduced their food supply. Almost all of them are now included in Nepal's nationally threatened list, for example Pallas's Fish Eagle Haliaeetus leucoryphus, Grey-headed Fish Eagle Ichthyophaga ichthyaetus, Brown Fish Owl Ketupa zeylonensis, Black-bellied Tern Sterna acuticauda, River Tern S. aurantia, and Indian Skimmer Rynchops albicollis.

Some important wetlands, for instance Jagdishpur Reservoir (which is a Ramsar site as well as an IBA) and Gaidahawa Tal, are contracted to commercial fishermen by the relevant authorities at each site, resulting in significant threats to wetland dependent birds. Disturbance caused by commercial fishermen results in birds flying away from relatively safe habitats and to sites where they are more at risk from hunting or trapping. The introduction of carnivorous fish species by the fishermen may impact on the survival of native fish ultimately affecting bird populations.

As a result of this onslaught of threats, a large percentage of Nepal's wetland birds (29 species, 25\%) is considered Critically Endangered or Endangered. Some wetland species have shown precipitous declines over recent years, for example Brahminy Kite Haliastur indus, Caspian Tern Sterna caspia, Black-bellied Tern S. acuticauda and River Tern S. aurantia (Fig. 5).

\section{Pressures on grassland birds}

A large percentage (23\%) of grassland birds is nationally threatened. The spread of cultivation in Nepal's lowlands has led to the once extensive lowland grasslands becoming greatly reduced and fragmented. Nepal's specialist grassland birds are now almost entirely confined to protected areas, where their populations are isolated (Baral 2001). Some sedentary species e.g. Slender-billed Babbler Turdoides longirostris and Jerdon's Babbler Chrysomma altirostre may therefore face long-term viability problems.

Within protected areas some bird species, for instance the globally threatened Bengal Florican Houbaropsis bengalensis, Swamp Francolin Francolinus gularis and White-throated Bushchat Saxicola insignis, are suffering from inappropriate grassland management, including ploughing, intensive annual cutting and burning, which alter grass species composition and are aimed the conservation of mammals, not birds (Baral 2001).

Lowland grasslands are traditionally exploited by local communities for their daily needs: grass for thatching roofs and weaving mats, as well as fodder for their livestock (Baral 2001). The Department of National Parks and Wildlife Conservation has struck a compromise between conservation and the survival needs of local people by allowing them to harvest grass for a limited period every year. As a result, 95\% of grassland is believed to be disturbed in Nepal's lowland protected areas during the grass-cutting season (H.S. Baral pers. obs. 2010).

Controlling illegal grazing and cutting activities is a difficult task for Nepal's park managers. Illegal overgrazing by livestock is by far the greatest threat to lowland grasslands in protected areas (Baral 2001); these domestic ungulates are at densities far higher than were formerly reached by wild species. Overall, a total of 14 species (61\%) of nationally threatened grassland species is in the Critically Endangered and Endangered categories.

Although upland grasslands occupy a far greater area than those in the lowlands, they support only two globally and nationally threatened species: Cheer Pheasant Catreus wallichii and Wood Snipe Gallinago nemoricola. Grasslands in the hills and mountains are both natural and human-made in origin. Above the tree line they are mainly naturally occurring alpine meadows up to the limit of vegetation. Below the tree line on south-facing slopes burning and livestock grazing, which mainly took place a very long time ago, have created and maintained grassy areas. These grasslands are poor in bird species diversity.

\section{Pressures on other specialist bird species}

Some nationally threatened birds have other specialist habitat needs and are now only locally distributed because of habitat losses.

Seven threatened species occur mainly in pure bamboo stands, for instance, Fulvous Parrotbill Paradoxornis fulvifrons and Golden-breasted Fulvetta Alcippe chrysotis. Eleven other species depend on or favour forests with a bamboo understorey, including Satyr Tragopan Tragopan satyra and Broad-billed Warbler Tickellia hodgsoni and are threatened to at least some degree by bamboo losses. Bamboo is a highly useful forest product; large quantities of bamboo Arundinaria spp. and Bambusa spp. are cut for weaving mats and baskets and for construction work. Overgrazing 


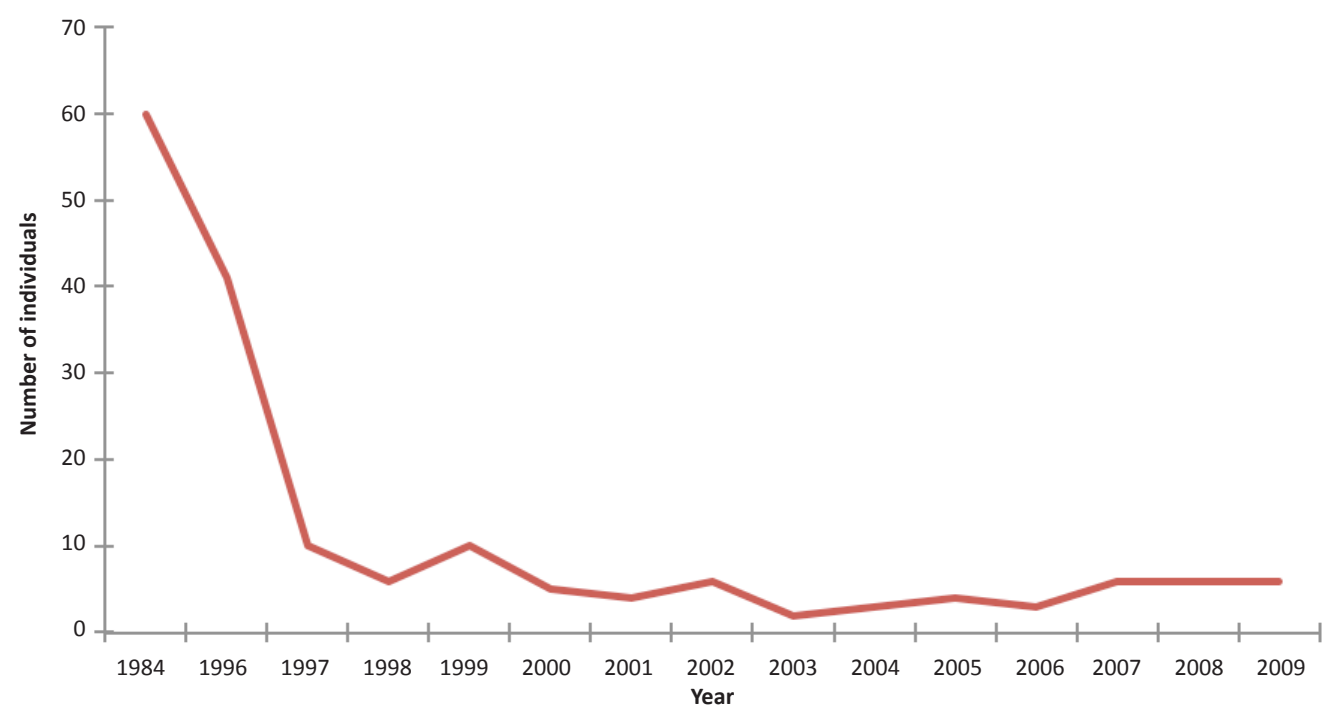

Figure 5. Numbers of Black-bellied Tern Sterna acuticauda recorded at Koshi 1996-2009

by livestock is also reducing bamboo in many areas e.g. Dhorpatan Hunting Reserve (Subedi 2008).

Great Hornbill Buceros bicornis and Great Slaty Woodpecker Mulleripicus pulverulentus require mature trees for feeding and nesting, but these are frequently and selectively felled as they are of high economic value.

\section{Hunting and trapping}

Around 43 nationally threatened species were found to be affected to some degree by hunting or trapping including the collection of nest contents $(29 \%$ of the total threatened). Recently two packs of feral dogs have been seen in Koshi Tappu Wildlife Reserve and feral dogs are known to threaten larger mammals in Bardia National Park, so they could also be a problem for birds. Wetland birds are especially at risk in all parts of Nepal. At Koshi, hunting and trapping birds for food and for sale at the market regularly takes place (Shakya 1995; Giri 2002). Bird hunting, including netting and egg collecting have also been identified as serious threats on Chitwan's rivers (Roberts et al. 2002; Tyabji 2002). Hunting is also threatening some grassland birds at risk, notably Swamp Francolin Francolinus gularis (Baral 1998), as well as some forest species, for instance Great Hornbill Buceros bicornis. A 2008/09 owl study in 22 out of Nepal's 75 districts revealed widespread hunting and trading of owls, especially Rock Eagle Owl Bubo bengalensis (Acharya \& Ghimirey 2009).

Pheasants are popular targets for hunters and trappers in some parts of Nepal. For example, hunting is a major threat to Cheer Pheasant Catreus wallichii in the upper Kali Gandaki valley, Annapurna Conservation
Area (Acharya et al. 2006) and in Rara National Park (Budhathapa 2006). However, hunting was not considered an important threat to Nepal's known key populations of Cheer Pheasant in and around Dhorpatan Hunting Reserve by Singh et al. (2006). The degree of threat caused by hunting and trapping often depends on traditional attitudes. For example Himalayan Monal Lophophorus impeyjanus is hardly hunted in Sagarmatha National Park where the large majority of the human population are Sherpas who are Buddhist and traditionally do not hunt. However, in Kanchenjunga Conservation Area the species is heavily hunted as the local people have hunted for generations and in an April 2008 survey, only one individual was located despite extensive suitable habitat (Inskipp et al. 2008).

\section{Impacts of alien weeds}

Serious threats are posed by some invasive alien weeds, notably Water-hyacinth Eichhornia crassipes, which was first reported in Nepal in 1966 and is now widely distributed in most protected areas ranging up to $1500 \mathrm{~m}$. Free-floating mats of Water-hyacinth in Koshi Tappu Wildlife Reserve have caused a sharp decline in the number of pure open water dwelling bird species, especially Oriental Darter Anhinga melanogaster, cormorants, and grebes, as well as reducing feeding areas for some ducks and other wetland birds (Dahal 2007).

More recently another alien, Bittervine Mikania micrantha, has invaded tropical and subtropical ecosystems from the far east to west-central Nepal (Siwakoti 2007) and has had devastating effects in some areas, notably in Chitwan National Park and Koshi Tappu 


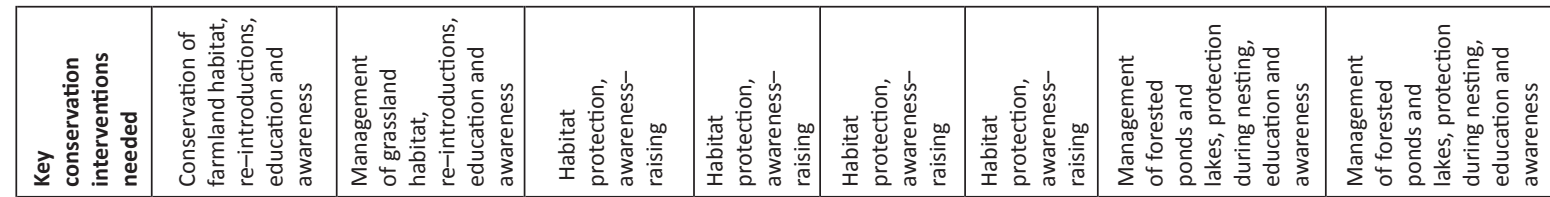

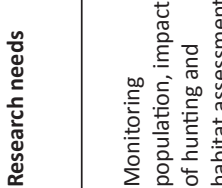

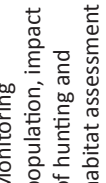

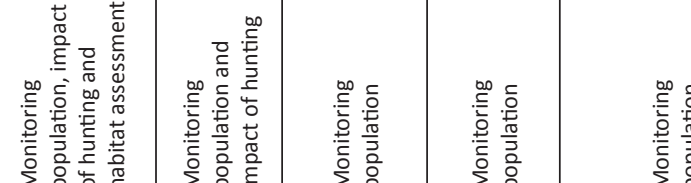

\begin{tabular}{|c|c|c|c|c|c|c|}
\hline 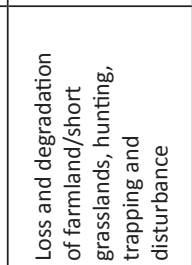 & 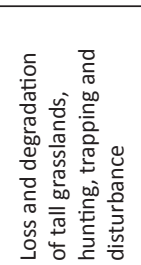 & 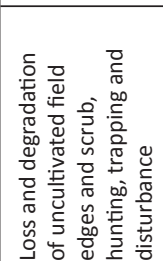 & 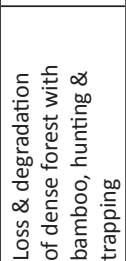 & 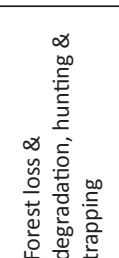 & 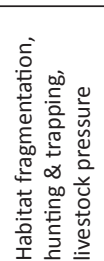 & 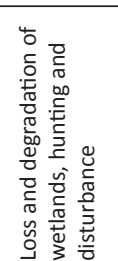 \\
\hline
\end{tabular}

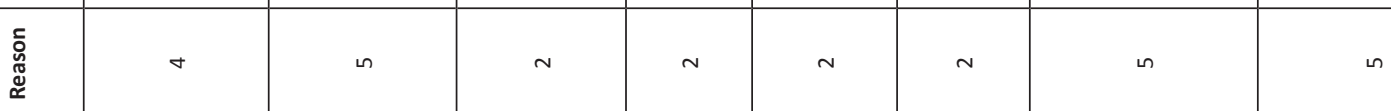

\begin{tabular}{|c|c|c|c|c|c|c|c|c|}
\hline 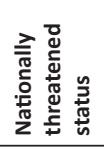 & 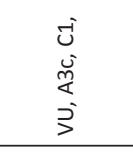 & 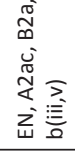 & 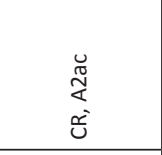 & 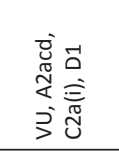 & 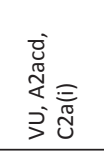 & 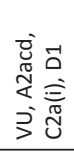 & 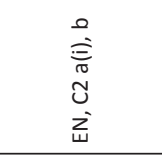 & $\begin{array}{l}\overrightarrow{0} \\
\dot{0} \\
\dot{3}\end{array}$ \\
\hline 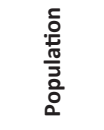 & & & & $\begin{array}{l}\stackrel{8}{ } \\
\vec{v}\end{array}$ & & $\begin{array}{l}\stackrel{8}{0} \\
\vec{v}\end{array}$ & 品 & $\begin{array}{l}\stackrel{8}{\Delta} \\
\vec{v}\end{array}$ \\
\hline 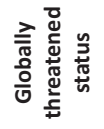 & & 3 & & 占 & & 3 & & \\
\hline 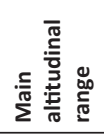 & 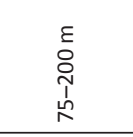 & 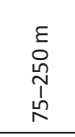 & 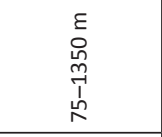 & 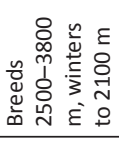 & 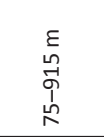 & 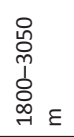 & $\begin{array}{l}\varepsilon \\
\stackrel{D}{N} \\
\stackrel{N}{\Lambda} \\
\end{array}$ & 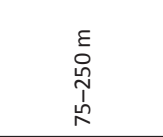 \\
\hline 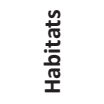 & U్ & 0 & ఝั & 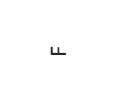 & ᄂ & цू & 3 & 3 \\
\hline 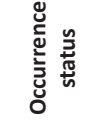 & $\propto$ & $\propto$ & $\propto$ & $\propto$ & $\propto$ & $\propto$ & $\propto$ & $\propto$ \\
\hline 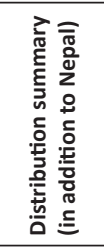 & 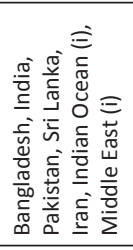 & 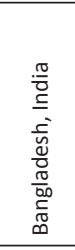 & 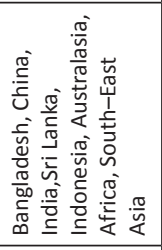 & 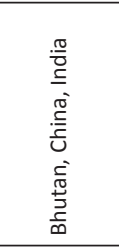 & 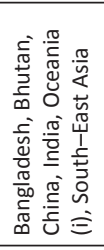 & 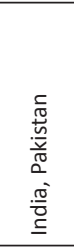 & 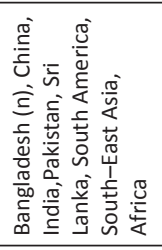 & 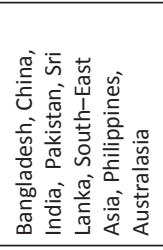 \\
\hline 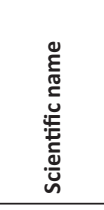 & 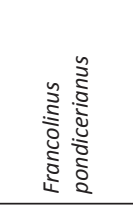 & 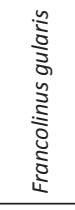 & 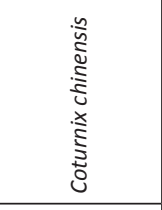 & 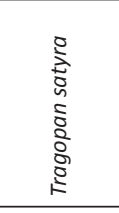 & 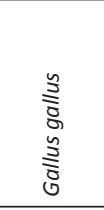 & 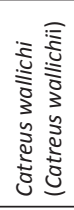 & 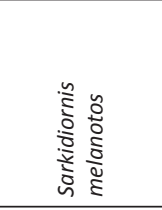 & 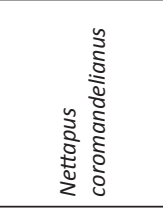 \\
\hline 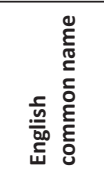 & 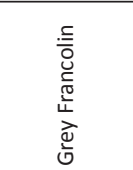 & 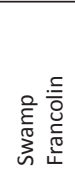 & 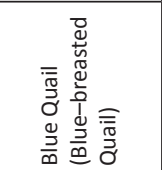 & 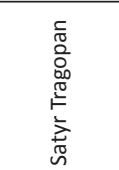 & 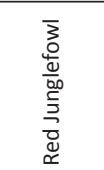 & 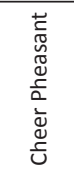 & $\begin{array}{l}\text { ․ㅡㅁ } \\
\text { D } \\
\text { है } \\
\text { है }\end{array}$ & 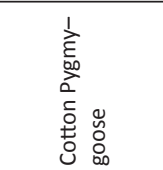 \\
\hline
\end{tabular}




\begin{tabular}{|c|c|c|c|c|c|c|c|c|c|}
\hline 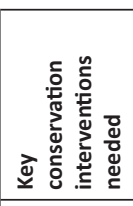 & 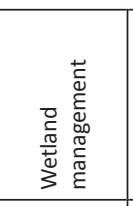 & 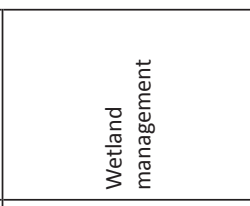 & 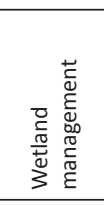 & 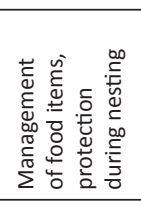 & 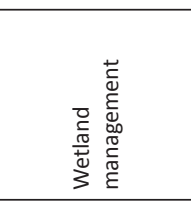 & 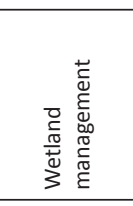 & 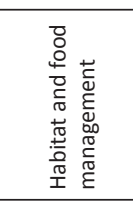 & 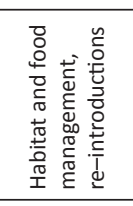 & 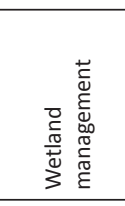 \\
\hline 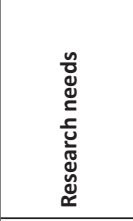 & 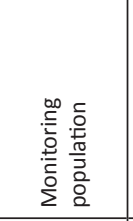 & 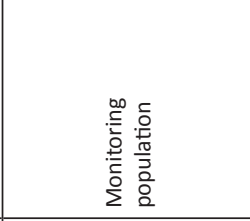 & 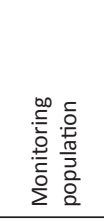 & 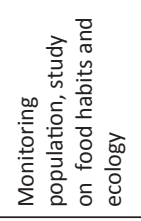 & 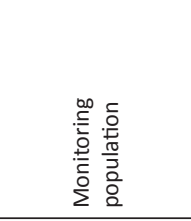 & 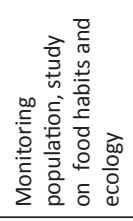 & 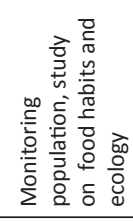 & 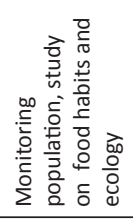 & 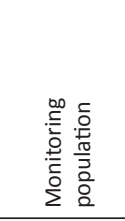 \\
\hline 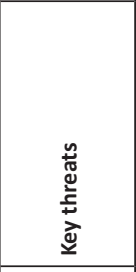 & 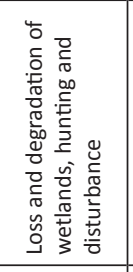 & 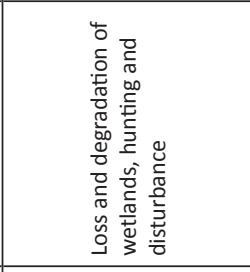 & 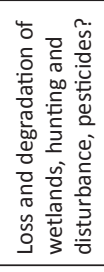 & 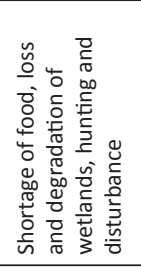 & 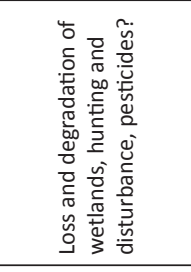 & 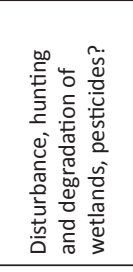 & 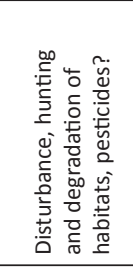 & 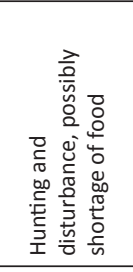 & 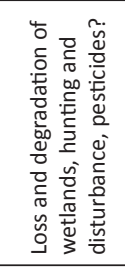 \\
\hline 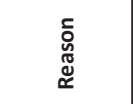 & $\stackrel{0}{-i}$ & in & in & $\triangleleft$ & in & $\stackrel{0}{i}$ & in & $\rightarrow$ & N \\
\hline 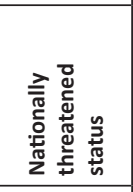 & 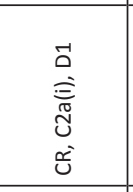 & $\begin{array}{l}5 \\
5 \\
0 \\
3 \\
3\end{array}$ & 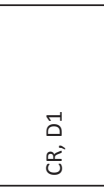 & $\begin{array}{l}\vec{J} \\
\vec{s}\end{array}$ & 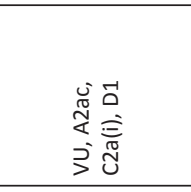 & $\begin{array}{l}\vec{a} \\
\text { ỗ } \\
\text { of }\end{array}$ & $\begin{array}{l}\bar{\Xi} \\
\tilde{N} \\
j \\
\end{array}$ & $\begin{array}{l}\overrightarrow{0} \\
\text { वे }\end{array}$ & 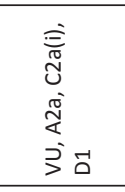 \\
\hline 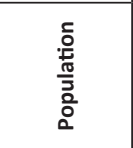 & 品 & $\underset{v}{o}$ & 号 & $\begin{array}{l}o \\
\stackrel{o}{v} \\
\vec{v}\end{array}$ & $\underset{\mathrm{v}}{\stackrel{\circ}{v}}$ & 品 & $\begin{array}{l}o \\
\text { v }\end{array}$ & $\stackrel{\leftrightarrow}{\vartheta}$ & $\underset{v}{\stackrel{O}{v}}$ \\
\hline 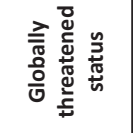 & 上 & s & 占 & & & 占 & 3 & z & z \\
\hline 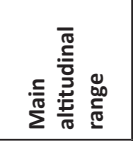 & 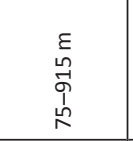 & 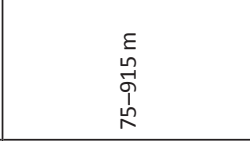 & 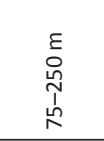 & 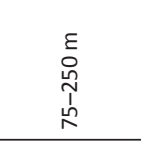 & 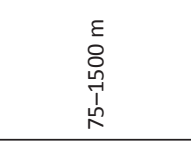 & 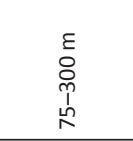 & 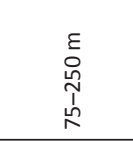 & 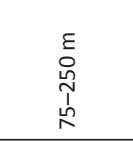 & 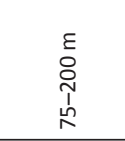 \\
\hline 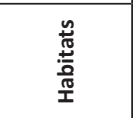 & 3 & 3 & 3 & 3 & 3 & 3 & 3 & 3 & 3 \\
\hline 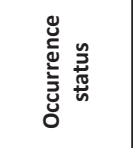 & $\xi$ & $\gtreqless$ & $\propto$ & $\propto$ & $\xi$ & $\propto$ & $\propto$ & $\frac{\infty}{z}$ & $\gtreqless$ \\
\hline 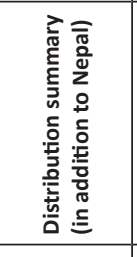 & 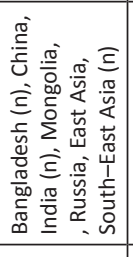 & 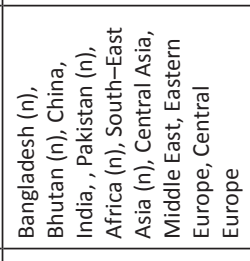 & 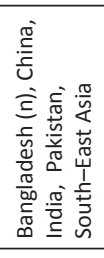 & 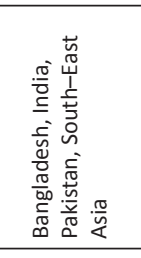 & 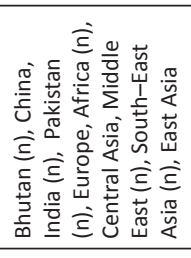 & 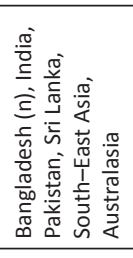 & 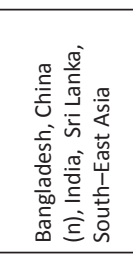 & 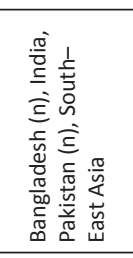 & 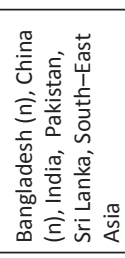 \\
\hline 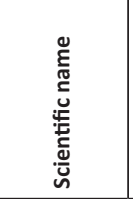 & 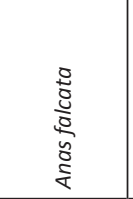 & 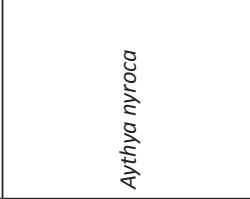 & 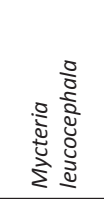 & 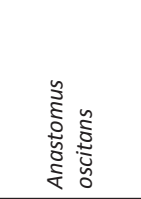 & 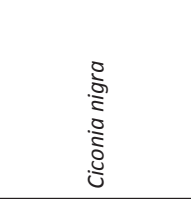 & 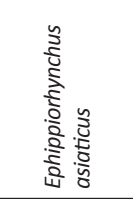 & 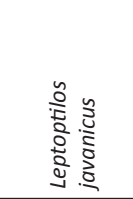 & 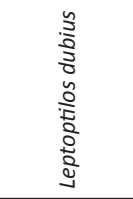 & 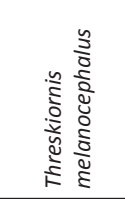 \\
\hline 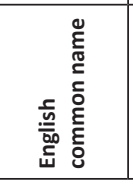 & 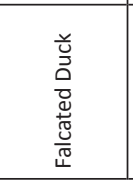 & 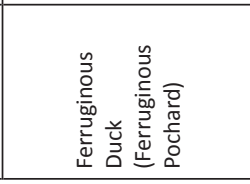 & 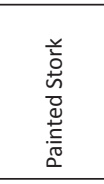 & 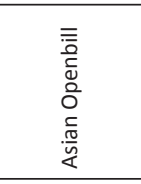 & 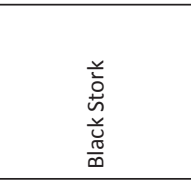 & 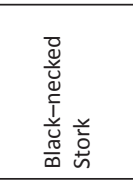 & 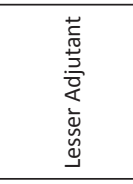 & 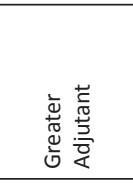 & 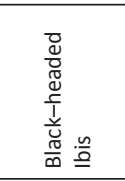 \\
\hline
\end{tabular}




\begin{tabular}{|c|c|c|c|c|c|c|c|c|}
\hline 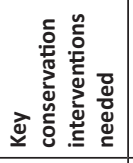 & 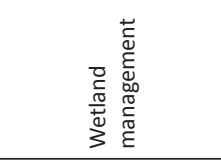 & 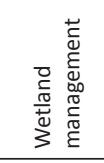 & 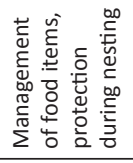 & 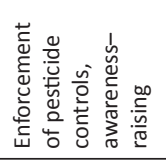 & 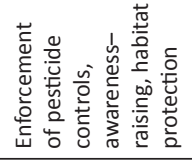 & 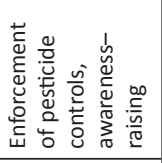 & 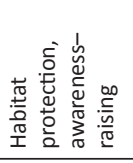 & 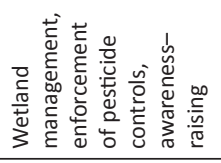 \\
\hline 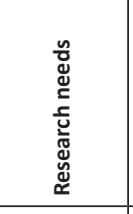 & 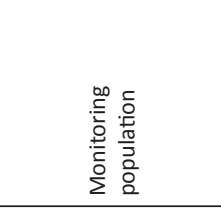 & 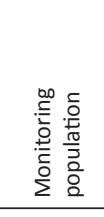 & 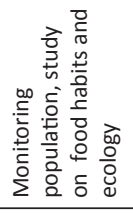 & 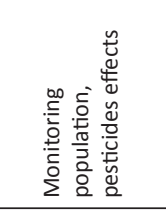 & 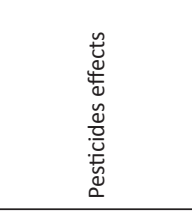 & 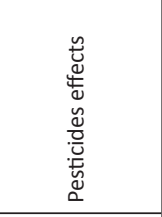 & 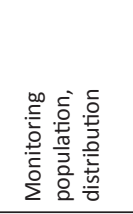 & 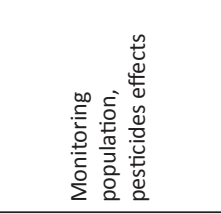 \\
\hline 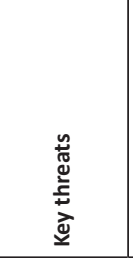 & 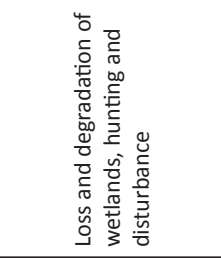 & 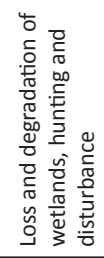 & 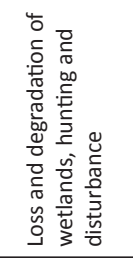 & 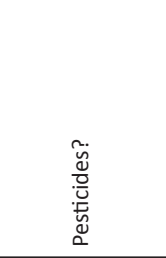 & 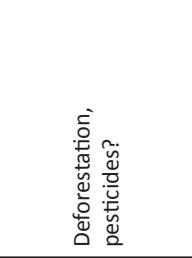 & 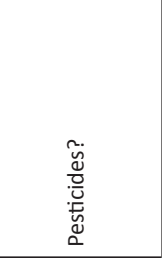 & 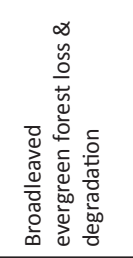 & 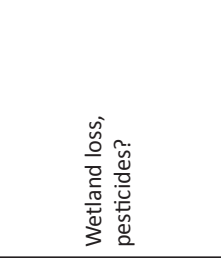 \\
\hline 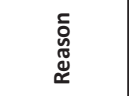 & $\sim$ & $\begin{array}{l}0 \\
\rightarrow\end{array}$ & in & 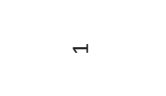 & 0 & 0 & 6 & $\underset{-}{0}$ \\
\hline 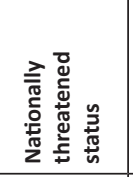 & 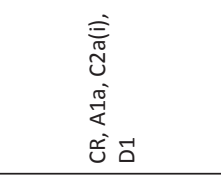 & 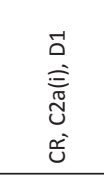 & 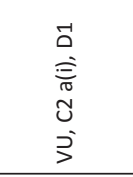 & 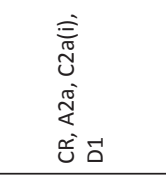 & $\begin{array}{l}\vec{a} \\
\text { ș }\end{array}$ & 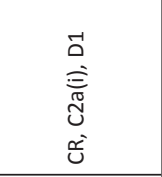 & 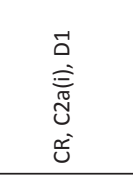 & 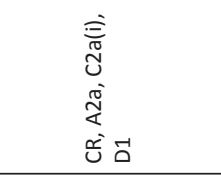 \\
\hline 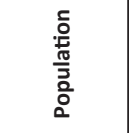 & 号 & 号 & $\begin{array}{l}\stackrel{O}{\vec{v}} \\
\text { the }\end{array}$ & 号 & 虽 & भु & $\stackrel{\leftrightarrow}{\leftrightarrow}$ & 号 \\
\hline 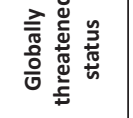 & & 之 & z & & & z & & \\
\hline 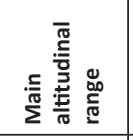 & $\begin{array}{l}\varepsilon \\
0 \\
\stackrel{N}{N} \\
\Lambda \\
N\end{array}$ & \begin{tabular}{l}
$\varepsilon$ \\
\multirow{\sigma}{0}{}
\end{tabular} & 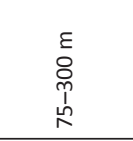 & $\begin{array}{l}\varepsilon \\
0 \\
\vdots \\
\vdots \\
1 \\
\end{array}$ & 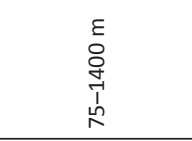 & 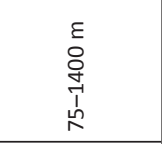 & 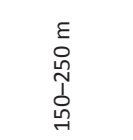 & 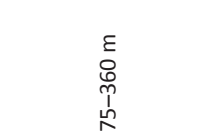 \\
\hline 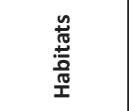 & 3 & 3 & 3 & ৪ & 3 & ৪ & 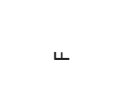 & 3 \\
\hline 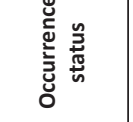 & $\xi$ & $\underline{m}$ & $\propto$ & $\propto$ & $\propto$ & $\simeq$ & $\propto$ & $\propto$ \\
\hline 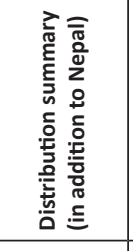 & 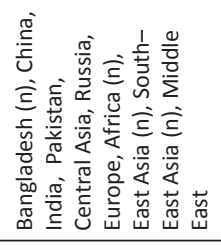 & 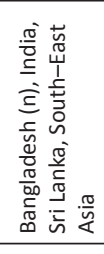 & 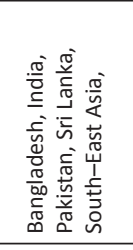 & 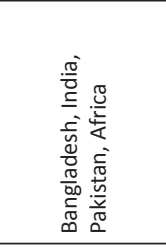 & 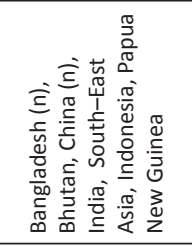 & 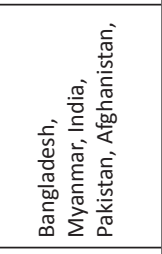 & 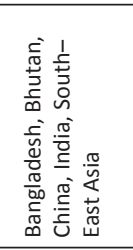 & 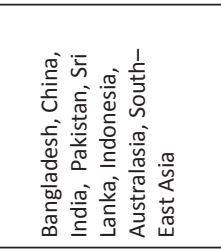 \\
\hline 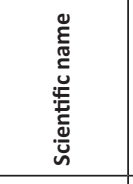 & 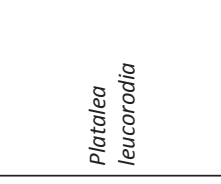 & 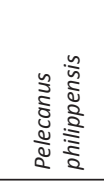 & 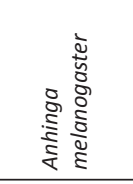 & 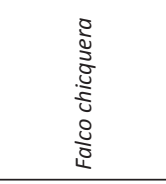 & 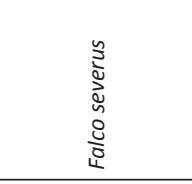 & 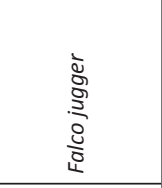 & 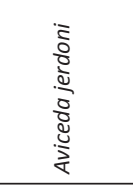 & 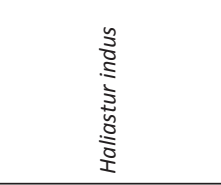 \\
\hline 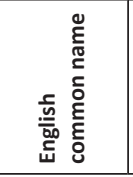 & 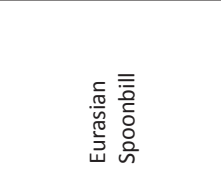 & 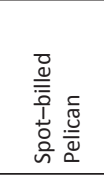 & 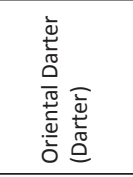 & 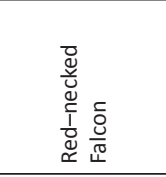 & 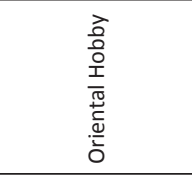 & 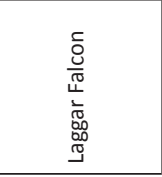 & 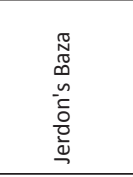 & 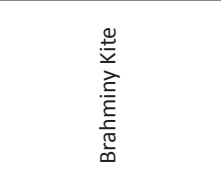 \\
\hline
\end{tabular}




\begin{tabular}{|c|c|c|c|c|c|c|}
\hline 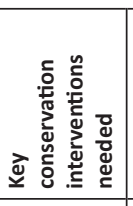 & 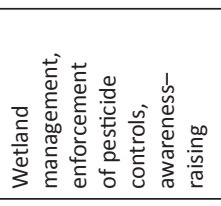 & 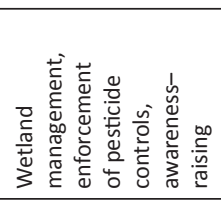 & 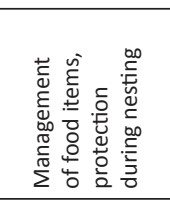 & 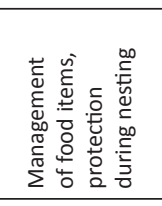 & 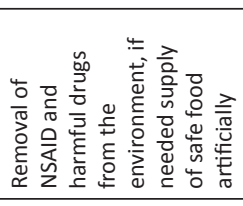 & 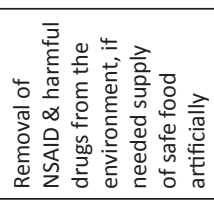 \\
\hline 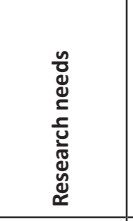 & 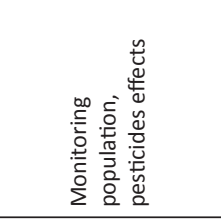 & 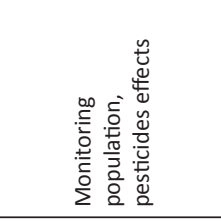 & 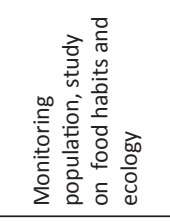 & 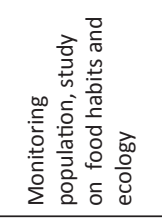 & 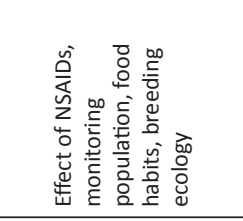 & 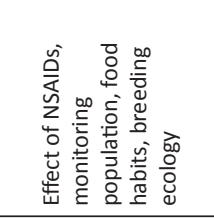 \\
\hline 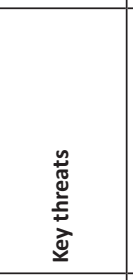 & 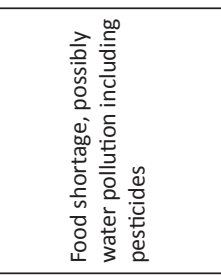 & 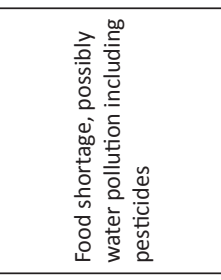 & 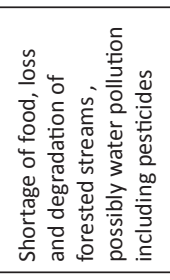 & 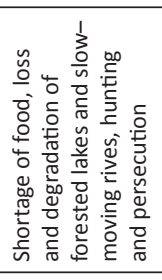 & 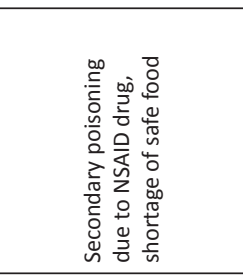 & 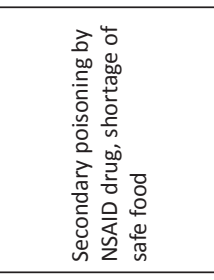 \\
\hline 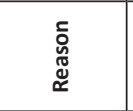 & 0 & 0 & n & 0 & $\stackrel{0}{\dot{m}^{-}}$ & $\sim$ \\
\hline 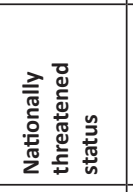 & 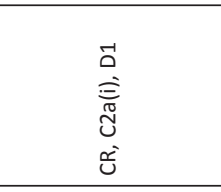 & 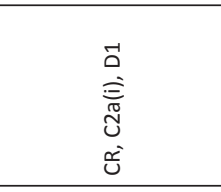 & 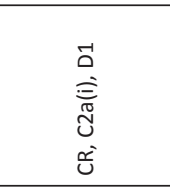 & 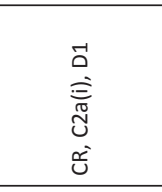 & 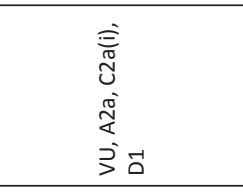 & 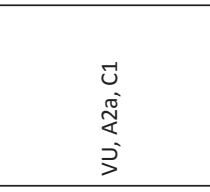 \\
\hline 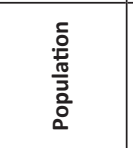 & 号 & 啘 & 号 & 虽 & 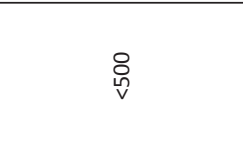 & $\underset{\vec{v}}{\stackrel{\circ}{ }}$ \\
\hline 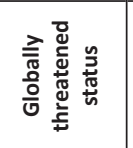 & З & & z & z & & z \\
\hline 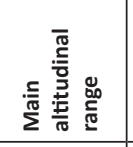 & $\begin{array}{l}\varepsilon \\
0 \\
0 \\
1 \\
n \\
\end{array}$ & 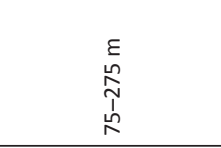 & $\begin{array}{l}\varepsilon \\
\underline{n} \\
\stackrel{9}{1} \\
\stackrel{1}{n} \\
\end{array}$ & 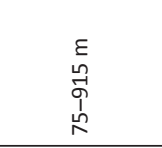 & 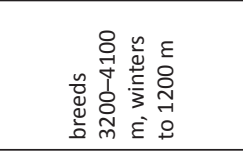 & 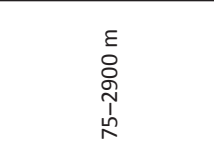 \\
\hline 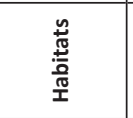 & 3 & 3 & 3 & 3 & ○ & 오 \\
\hline 营 & $\xi$ & $\xi$ & $\propto$ & $\propto$ & $\propto$ & $\propto$ \\
\hline 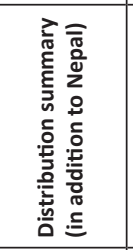 & 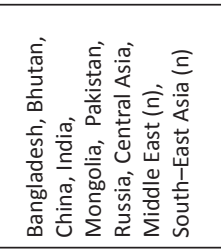 & 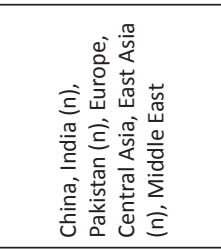 & 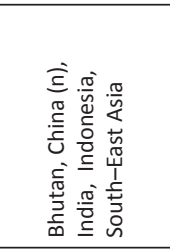 & 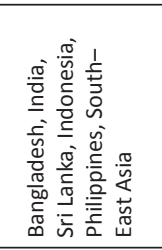 & 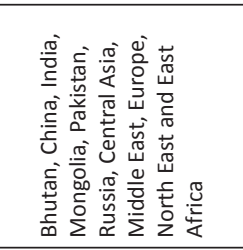 & 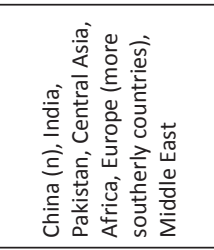 \\
\hline 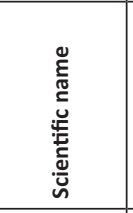 & 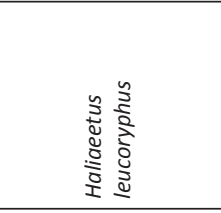 & 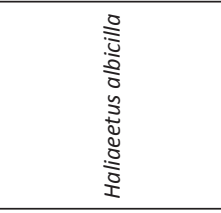 & 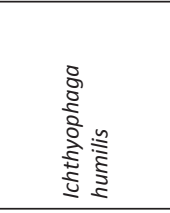 & 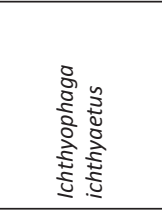 & 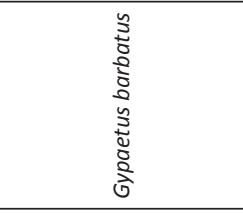 & 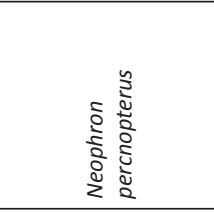 \\
\hline 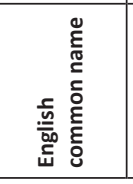 & 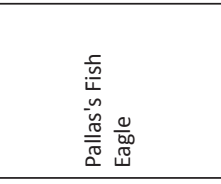 & 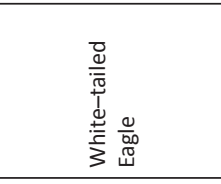 & 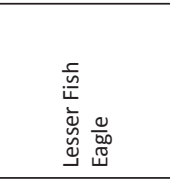 & 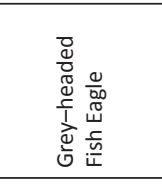 & 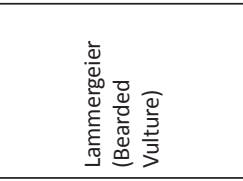 & 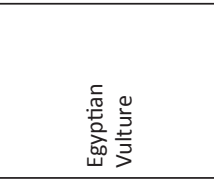 \\
\hline
\end{tabular}




\begin{tabular}{|c|c|c|c|c|c|}
\hline 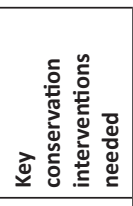 & 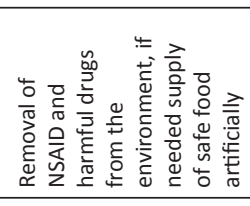 & 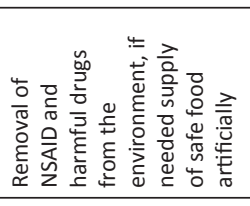 & 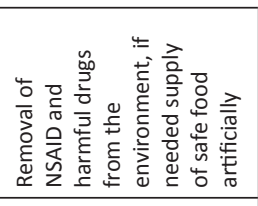 & 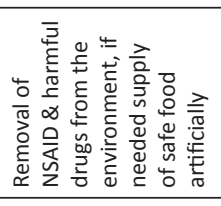 & 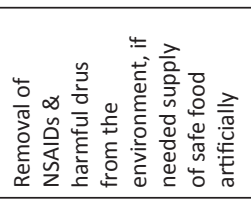 \\
\hline 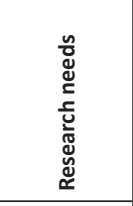 & 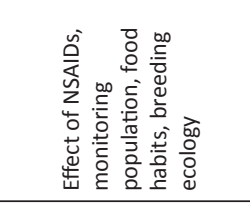 & 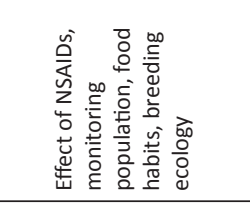 & 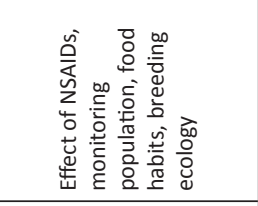 & 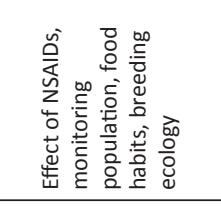 & 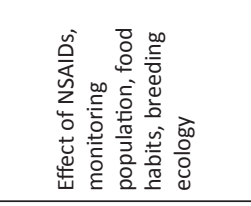 \\
\hline 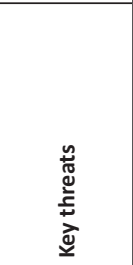 & 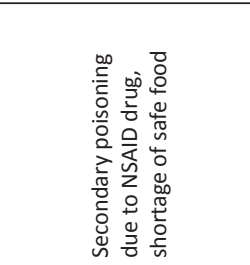 & 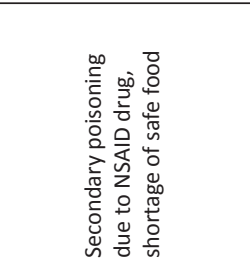 & 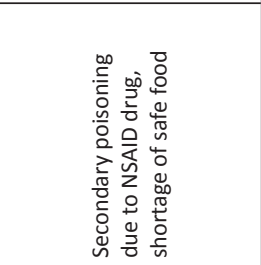 & 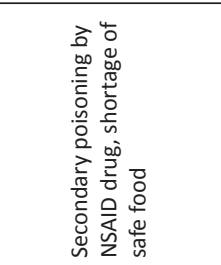 & 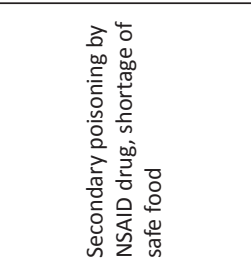 \\
\hline 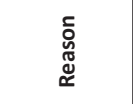 & 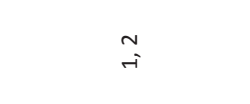 & $\stackrel{\sim}{\stackrel{\sim}{\sim}}$ & $\underset{m}{i}$ & N & $\sim$ \\
\hline 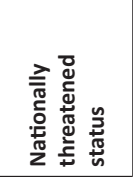 & 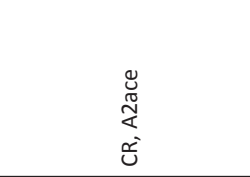 & 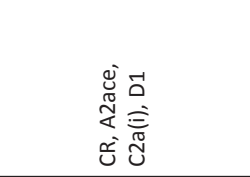 & 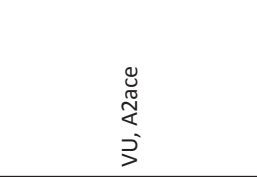 & 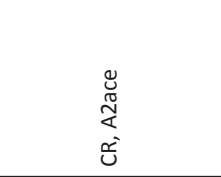 & 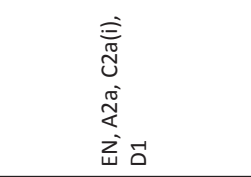 \\
\hline 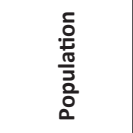 & : & $\stackrel{\leftrightarrow}{\leftrightarrow}$ & 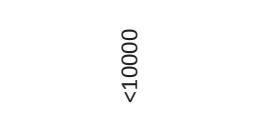 & 各 & 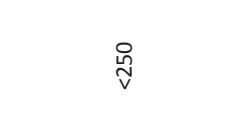 \\
\hline 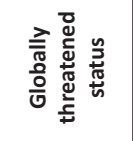 & 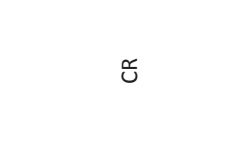 & ㅁ & & $\stackrel{\simeq}{\cup}$ & 5 \\
\hline 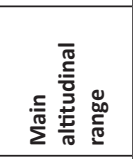 & $\begin{array}{l}\varepsilon \\
0 \\
0 \\
0 \\
1 \\
\\
\end{array}$ & 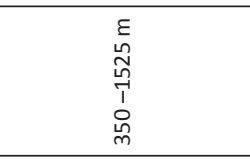 & 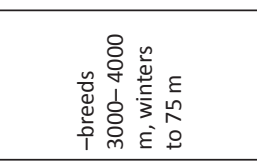 & 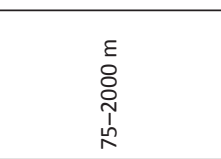 & 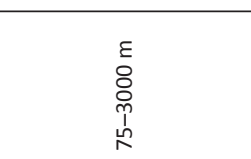 \\
\hline 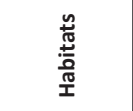 & I & I & ० & 요 & $\circ$ \\
\hline 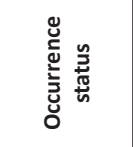 & $\propto$ & $\propto$ & $\propto$ & $\propto$ & $\xi$ \\
\hline 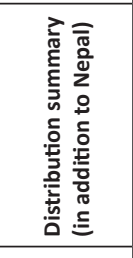 & 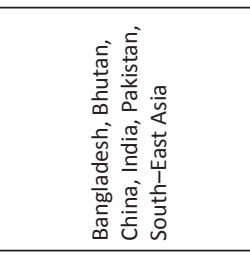 & 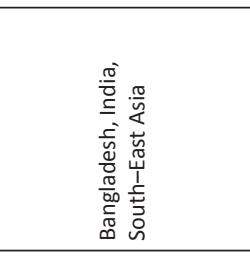 & 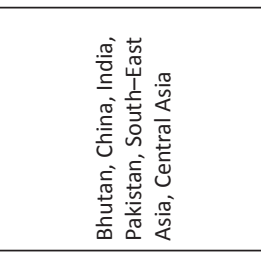 & 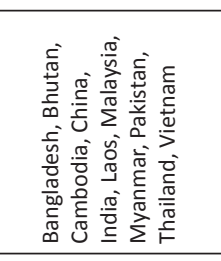 & 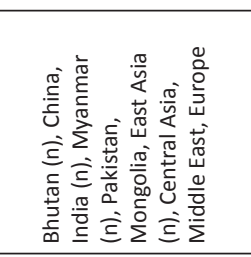 \\
\hline 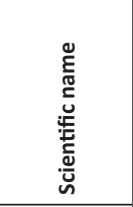 & 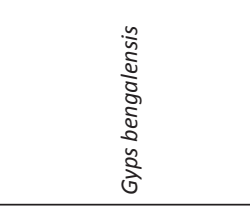 & 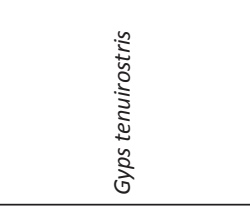 & 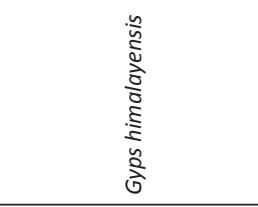 & 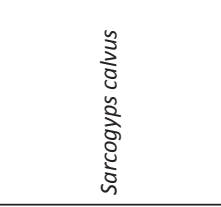 & 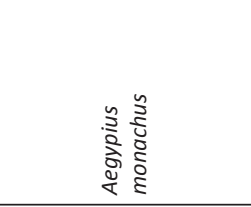 \\
\hline 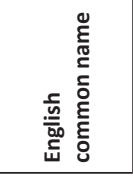 & 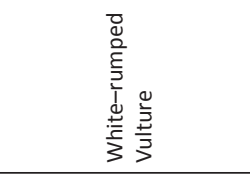 & 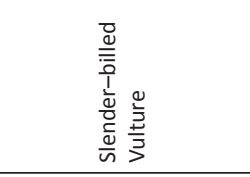 & 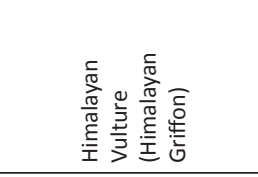 & 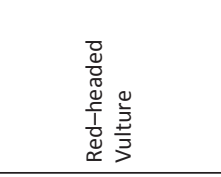 & 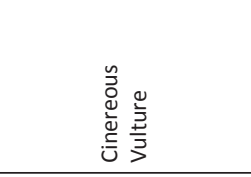 \\
\hline
\end{tabular}




\begin{tabular}{|c|c|c|c|c|c|c|}
\hline 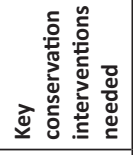 & $\approx$ & $\approx:$ & $\approx:$ & 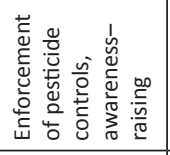 & 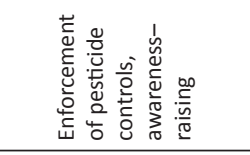 & 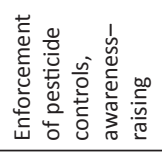 \\
\hline 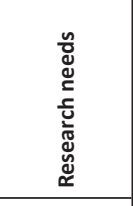 & 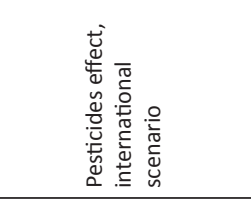 & 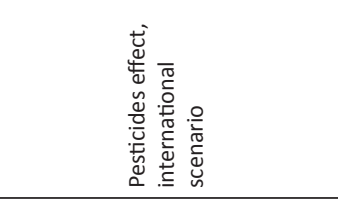 & 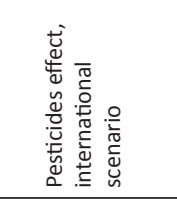 & 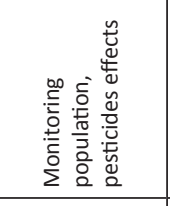 & 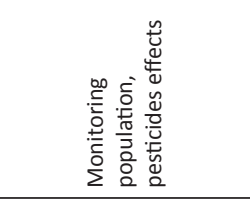 & 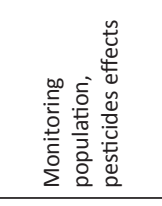 \\
\hline 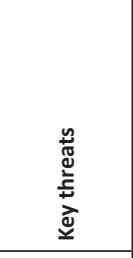 & 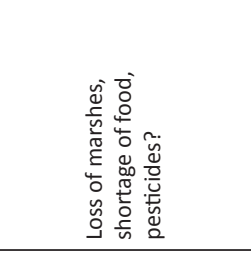 & 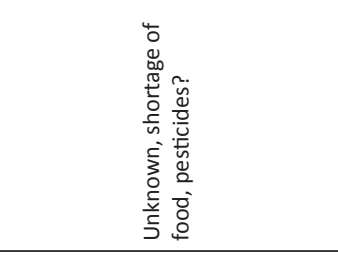 & 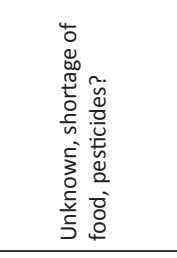 & 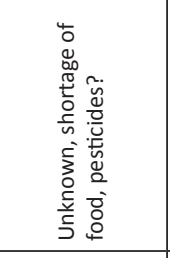 & 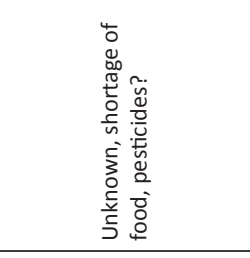 & 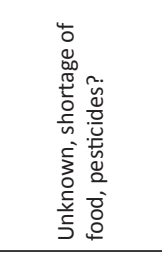 \\
\hline $\begin{array}{l}\bar{\delta} \\
\stackrel{d}{\mathbb{d}} \\
\approx\end{array}$ & $\sim$ & $\sim$ & $\sim$ & $\bullet$ & 0 & $\stackrel{i}{i}$ \\
\hline 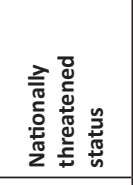 & 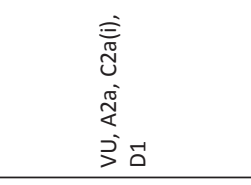 & 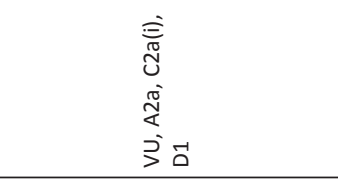 & 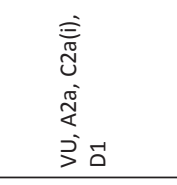 & 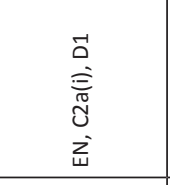 & $\begin{array}{l}\overrightarrow{0} \\
\stackrel{\bar{\Xi}}{\pi} \\
\widetilde{0} \\
\text { ż } \\
\end{array}$ & 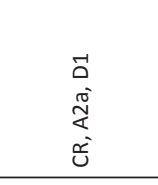 \\
\hline 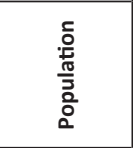 & $\underset{\vec{v}}{\stackrel{\leftrightarrow}{ }}$ & 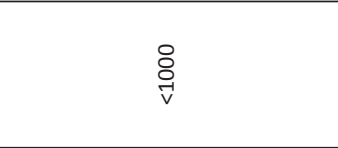 & $\underset{\vec{v}}{\stackrel{\circ}{ }}$ & $\stackrel{\stackrel{Q}{N}}{v}$ & $\stackrel{o}{\vec{v}}$ & $\stackrel{p}{v}$ \\
\hline 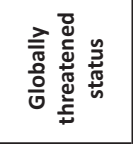 & & & & 3 & 3 & \\
\hline 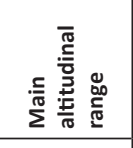 & 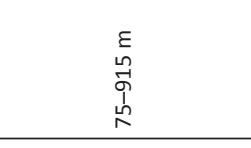 & $\begin{array}{l}\varepsilon \\
0 \\
0 \\
0 \\
\omega \\
\\
\end{array}$ & 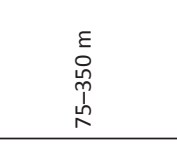 & 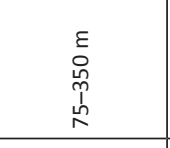 & 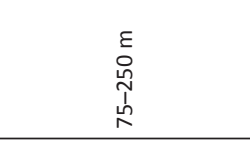 & 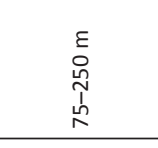 \\
\hline 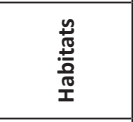 & 3 & 0 & o & 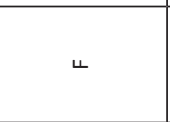 & 3 & ৪ \\
\hline 总 & $\gtreqless$ & $\xi$ & $\gtreqless$ & $\propto$ & $\xi$ & $\propto$ \\
\hline 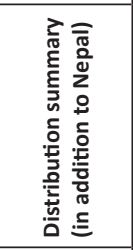 & 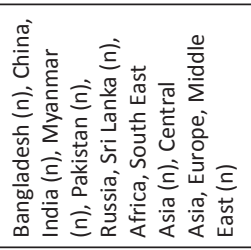 & 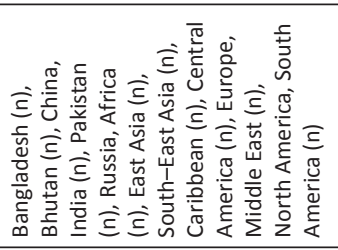 & 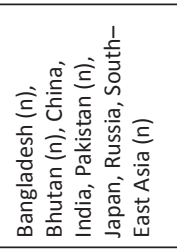 & 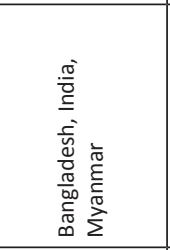 & 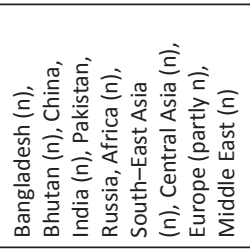 & 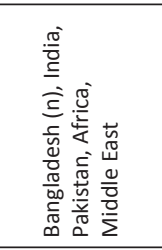 \\
\hline 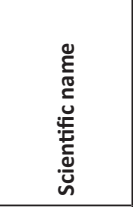 & 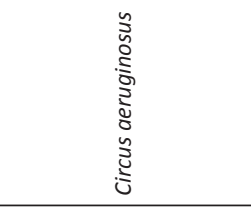 & 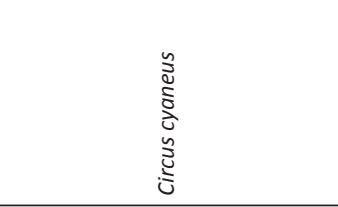 & 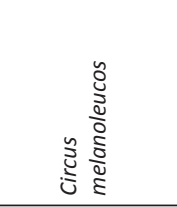 & 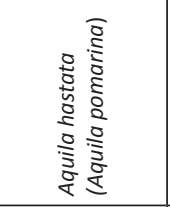 & 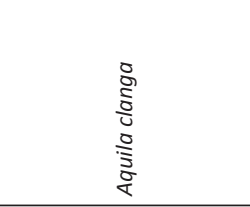 & 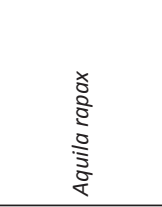 \\
\hline 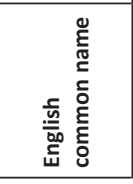 & 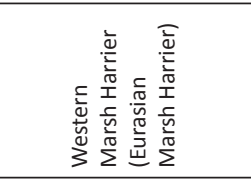 & 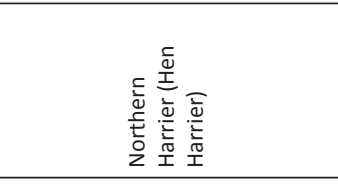 & 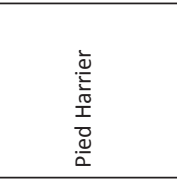 & 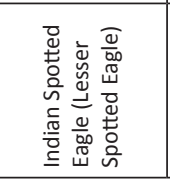 & 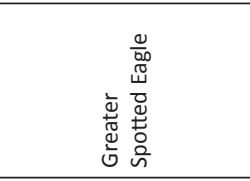 & 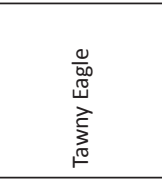 \\
\hline
\end{tabular}




\begin{tabular}{|c|c|c|c|c|c|c|c|c|}
\hline 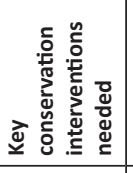 & & 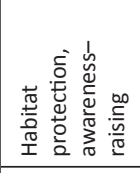 & 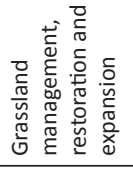 & 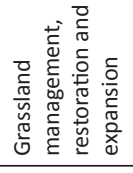 & 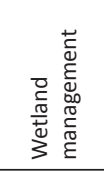 & 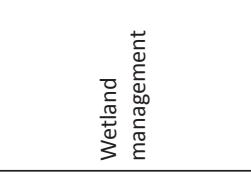 & 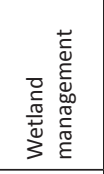 & 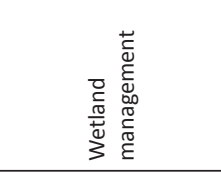 \\
\hline 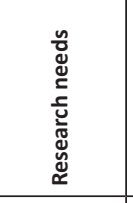 & 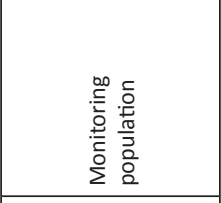 & 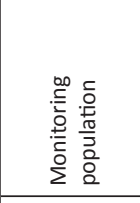 & 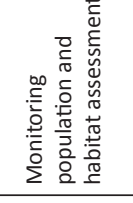 & 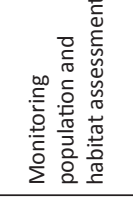 & 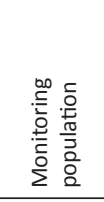 & 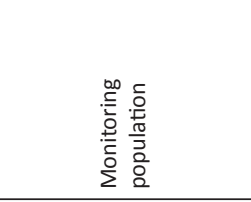 & 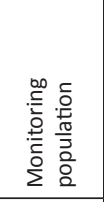 & 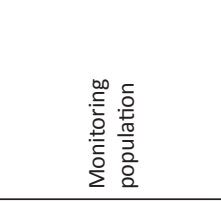 \\
\hline 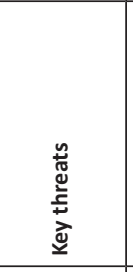 & 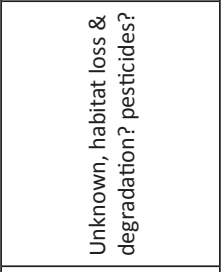 & 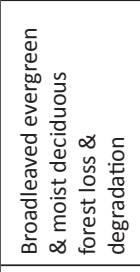 & 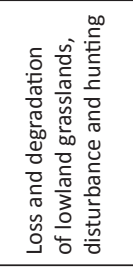 & 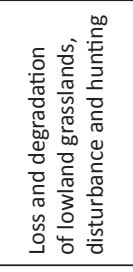 & 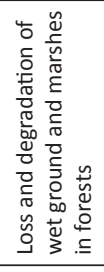 & 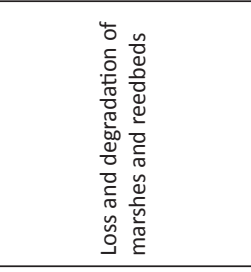 & 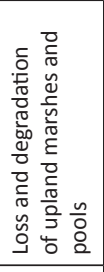 & 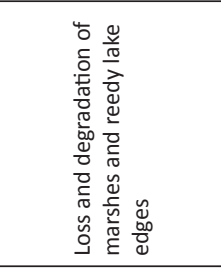 \\
\hline 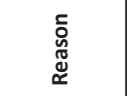 & 0 & in & 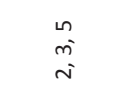 & $\stackrel{0}{\rightarrow}$ & 0 & in & $\stackrel{n}{i}$ & $\stackrel{n}{i}$ \\
\hline 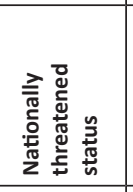 & 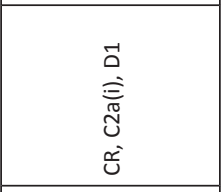 & 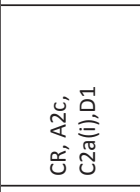 & 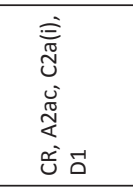 & 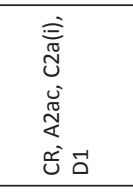 & $\begin{array}{l}\overrightarrow{0} \\
\text { 产 } \\
\tilde{N} \\
\text { ż } \\
\end{array}$ & 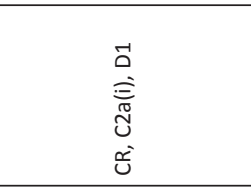 & $\begin{array}{l}\overrightarrow{0} \\
\stackrel{\bar{\Xi}}{\sigma} \\
\tilde{O} \\
\dot{u} \\
\end{array}$ & 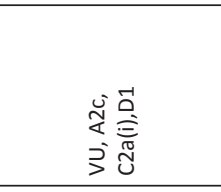 \\
\hline 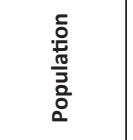 & $\stackrel{\circ}{\text { Q }}$ & 号 & 虽 & ஜٌ & $\stackrel{\stackrel{D}{\sim}}{v}$ & 品 & 咨 & 品 \\
\hline 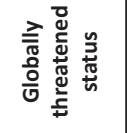 & 3 & & 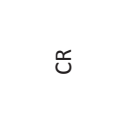 & z & & & & \\
\hline 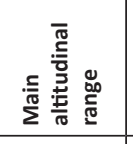 & $\begin{array}{l}\varepsilon \\
\stackrel{1}{0} \\
\end{array}$ & 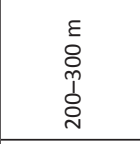 & 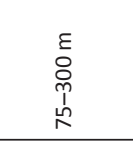 & $\begin{array}{l}\varepsilon \\
\vdots \\
\vdots \\
\vdots \\
\\
\end{array}$ & $\begin{array}{l}E \\
\text { 品 } \\
\end{array}$ & $\begin{array}{l}E \\
0 \\
\vdots \\
\vdots \\
1 \\
\end{array}$ & $\begin{array}{l}E \\
\stackrel{్}{N} \\
\stackrel{్}{J}\end{array}$ & 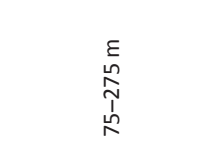 \\
\hline $\begin{array}{l}\frac{n}{5} \\
\frac{50}{\pi 0} \\
\frac{5}{x}\end{array}$ & 3 & 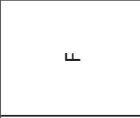 & ৩ & ० & 3 & 3 & 3 & 3 \\
\hline 莺 & $\gtreqless$ & $\propto$ & $\propto$ & in & $\propto$ & $\gtreqless$ & $\propto$ & $\gtreqless$ \\
\hline 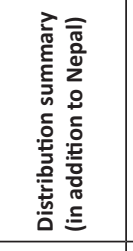 & 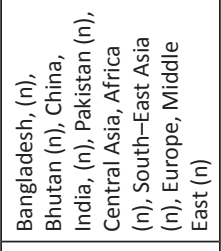 & 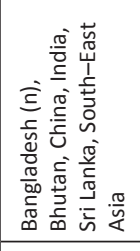 & 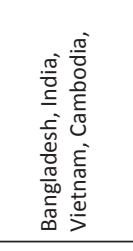 & 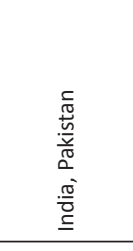 & 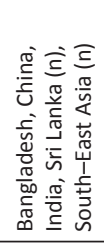 & 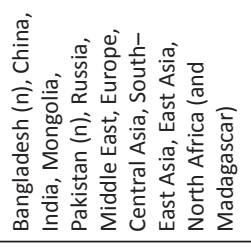 & 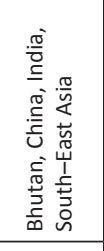 & 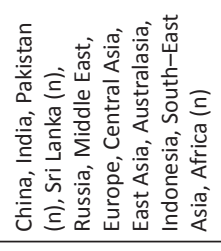 \\
\hline 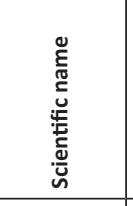 & 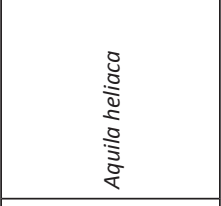 & 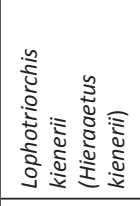 & 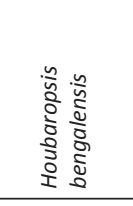 & 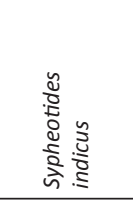 & 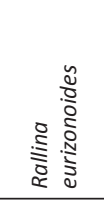 & 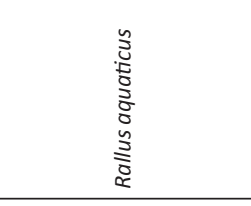 & 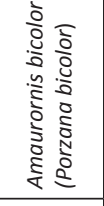 & $\begin{array}{l}\frac{1}{5} \\
\frac{5}{3} \\
0 \\
0 \\
0 \\
0 \\
0 \\
0 \\
\end{array}$ \\
\hline 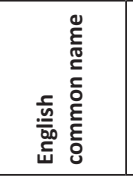 & 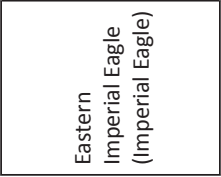 & 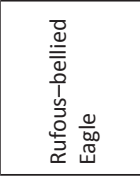 & 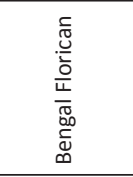 & 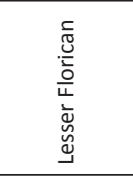 & 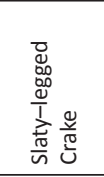 & 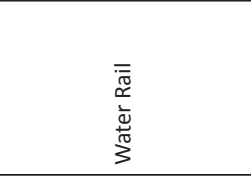 & 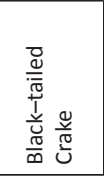 & 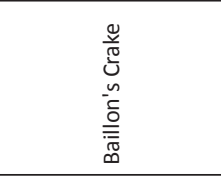 \\
\hline
\end{tabular}




\begin{tabular}{|c|c|c|c|c|c|c|c|c|}
\hline 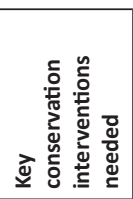 & 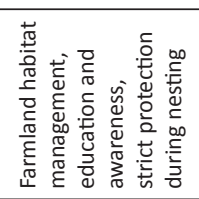 & 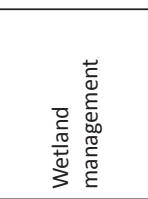 & 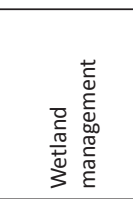 & 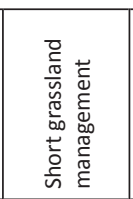 & 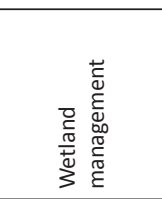 & 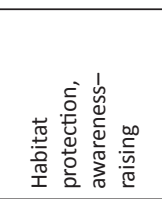 & 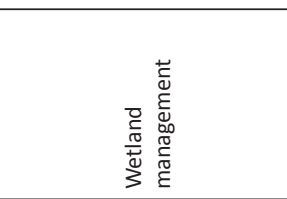 & 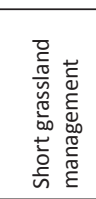 \\
\hline 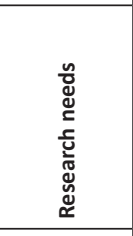 & 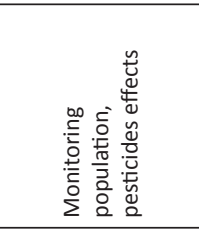 & 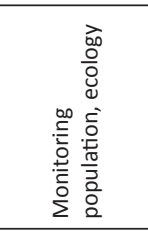 & 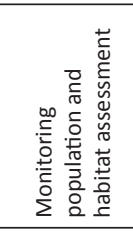 & 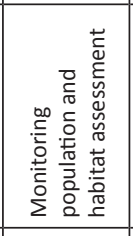 & 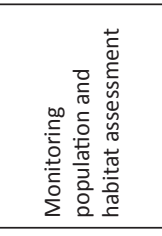 & 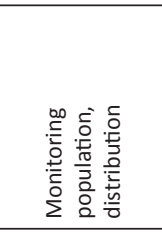 & 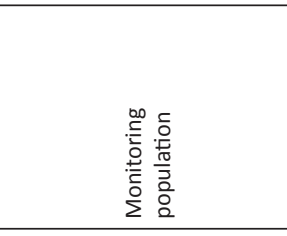 & 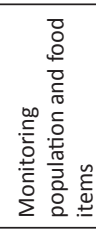 \\
\hline 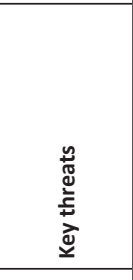 & 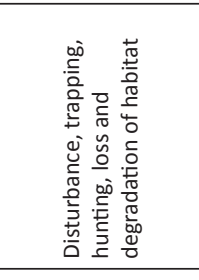 & 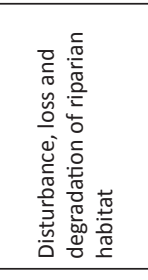 & 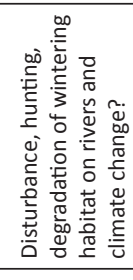 & 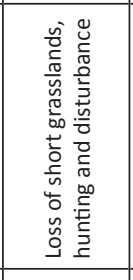 & 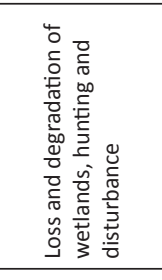 & 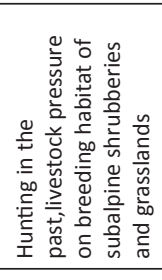 & 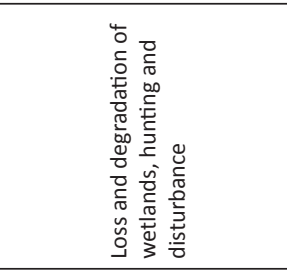 & 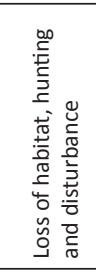 \\
\hline 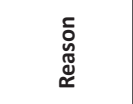 & $\begin{array}{l}n \\
\stackrel{n}{+} \\
i\end{array}$ & $\stackrel{n}{i}$ & $\stackrel{n}{i}$ & 6 & in & -1 & $\stackrel{n}{i}$ & n \\
\hline 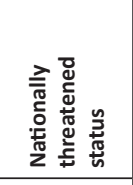 & $\begin{array}{l}\bar{\Xi} \\
\tilde{J} \\
z \\
\text { z } \\
\end{array}$ & 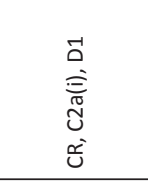 & 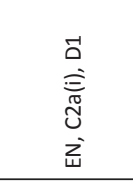 & 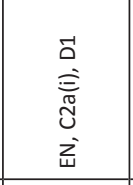 & 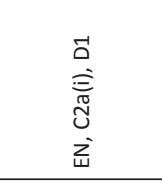 & 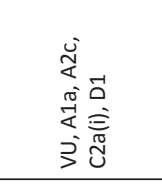 & 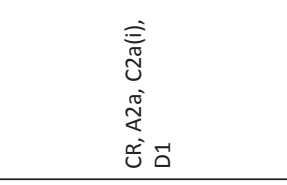 & $\begin{array}{l}\bar{\Xi} \\
\tilde{J} \\
z \\
\text { i } \\
\end{array}$ \\
\hline \begin{tabular}{l}
\multicolumn{2}{c}{} \\
$\stackrel{\circ}{0}$ \\
$\frac{\pi}{3}$ \\
$\frac{0}{0}$ \\
0
\end{tabular} & 吕 & 号 & $\stackrel{\circ}{\sim}$ & $\stackrel{\stackrel{O}{\mathrm{v}}}{\mathrm{v}}$ & $\stackrel{\stackrel{\leftrightarrow}{v}}{v}$ & 号 & 号 & $\underset{v}{\stackrel{\circ}{v}}$ \\
\hline 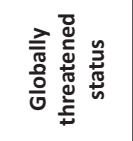 & 3 & & & & & 3 & z & \\
\hline 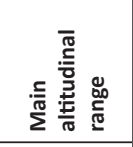 & 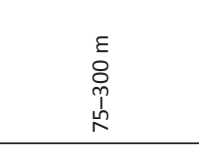 & $\begin{array}{l}\varepsilon \\
\text { E } \\
\text { N } \\
\text { L } \\
\end{array}$ & 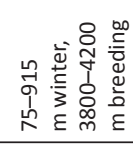 & 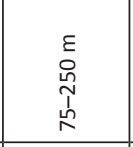 & 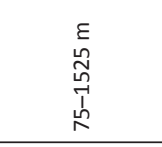 & 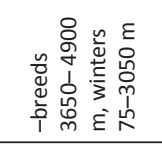 & $\underset{\square}{\varepsilon}$ & 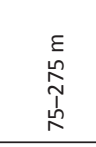 \\
\hline $\begin{array}{l}\stackrel{5}{5} \\
\stackrel{5}{0} \\
\text { I5 } \\
\text { I5 }\end{array}$ & $u$ & 3 & 3 & ঃ & 3 & 妾 & 3 & $\circ$ \\
\hline 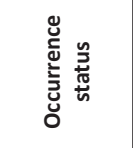 & $\propto$ & $\propto$ & $\propto$ & $\propto$ & $\propto$ & $\vec{n}$ & $\xi$ & $\propto$ \\
\hline 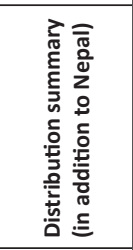 & 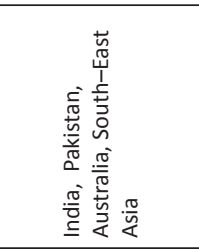 & 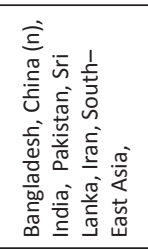 & 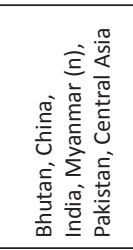 & 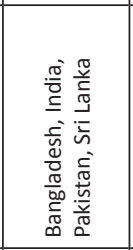 & 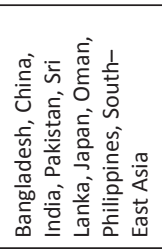 & 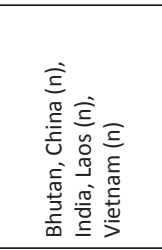 & 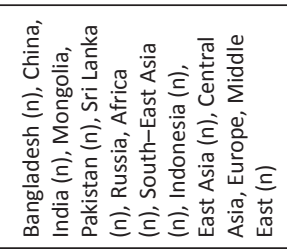 & 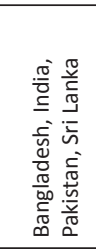 \\
\hline 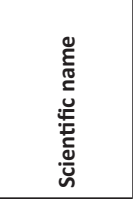 & 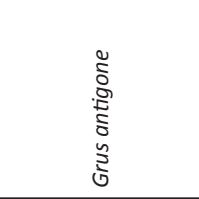 & 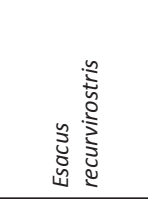 & 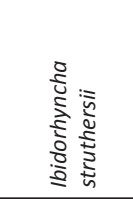 & 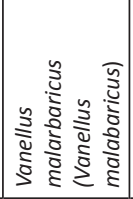 & 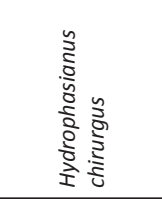 & 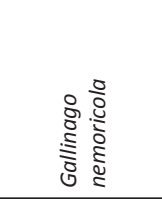 & 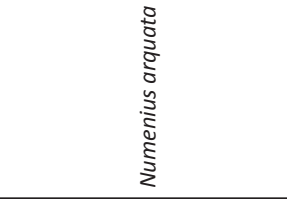 & 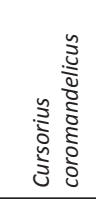 \\
\hline 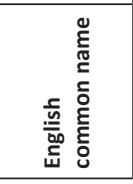 & 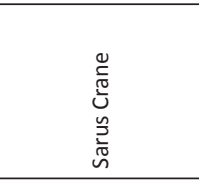 & 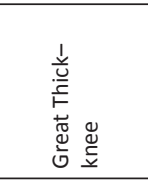 & $\begin{array}{l}\overline{\overline{\overline{0}}} \\
\underline{\underline{\underline{\underline{n}}}} \\
\end{array}$ & 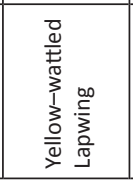 & 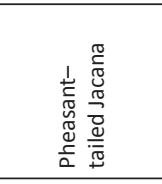 & 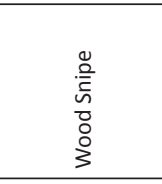 & 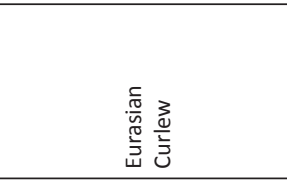 & 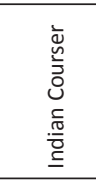 \\
\hline
\end{tabular}




\begin{tabular}{|c|c|c|c|c|c|c|c|}
\hline 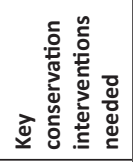 & 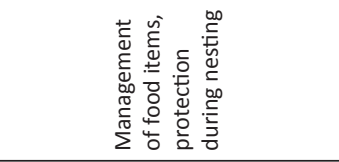 & 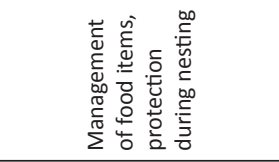 & 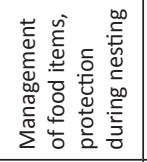 & 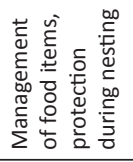 & 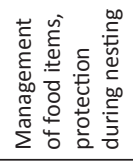 & 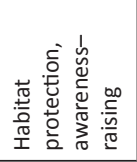 & 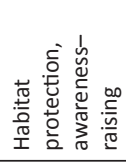 \\
\hline 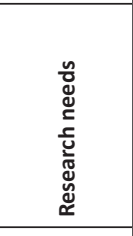 & 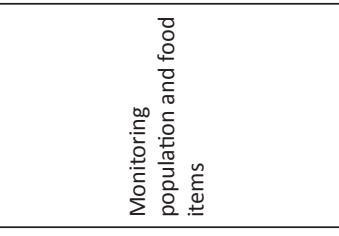 & 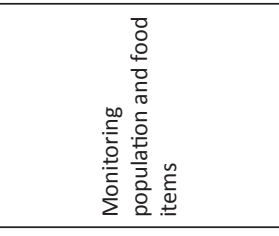 & 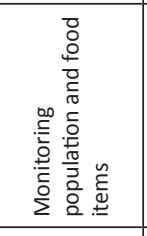 & 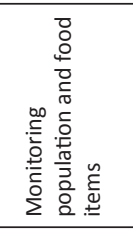 & 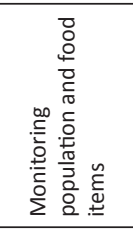 & 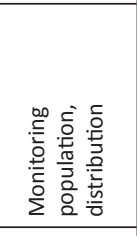 & 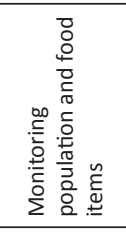 \\
\hline 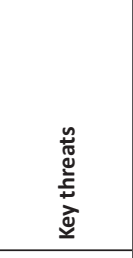 & 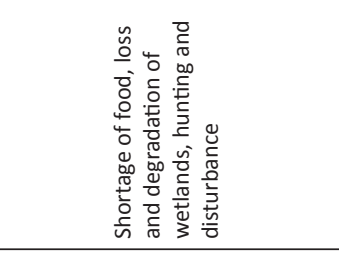 & 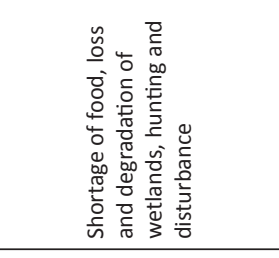 & 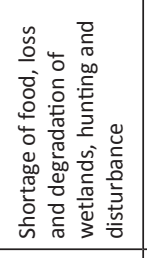 & 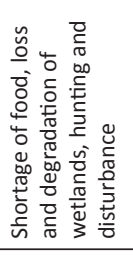 & 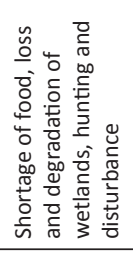 & 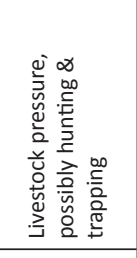 & 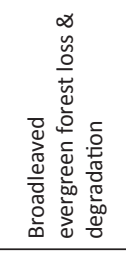 \\
\hline 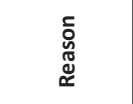 & $\begin{array}{l}0 \\
\stackrel{i}{i} \\
i\end{array}$ & $\begin{array}{l}0 \\
i \\
i\end{array}$ & $\begin{array}{l}0 \\
i \\
i \\
i\end{array}$ & $\begin{array}{l}0 \\
i \\
i \\
i\end{array}$ & $\begin{array}{l}0 \\
\stackrel{1}{*} \\
i\end{array}$ & $\begin{array}{l}0 \\
\sim \\
\sim\end{array}$ & $\sim$ \\
\hline 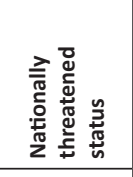 & 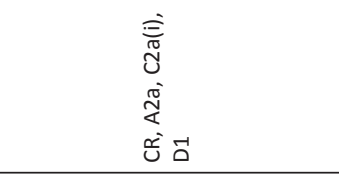 & 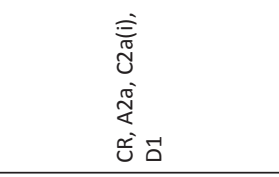 & 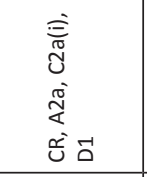 & 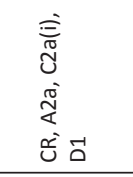 & 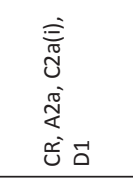 & 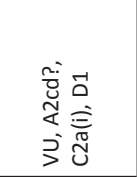 & 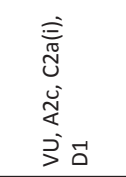 \\
\hline 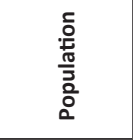 & 品 & 号 & 品 & 号 & 号 & 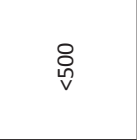 & $\begin{array}{l}\stackrel{O}{O} \\
\vec{v}\end{array}$ \\
\hline 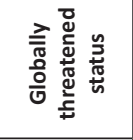 & & & & z & 3 & & \\
\hline 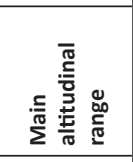 & $\begin{array}{l}\varepsilon \\
\stackrel{-}{0} \\
\end{array}$ & 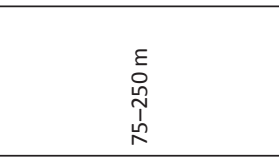 & 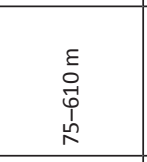 & 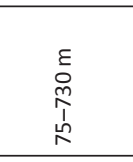 & 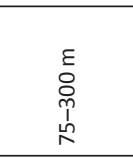 & 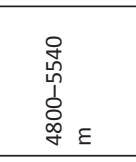 & $\begin{array}{l}\varepsilon \\
\stackrel{\varepsilon}{0} \\
\stackrel{0}{N} \\
\vdots \\
o \\
0\end{array}$ \\
\hline 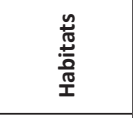 & 3 & 3 & 3 & 3 & 3 & ○ & 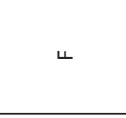 \\
\hline 总 & $\xi$ & $\gtreqless$ & $\propto$ & $\propto$ & 永 & $\propto$ & $\propto$ \\
\hline 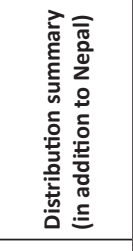 & 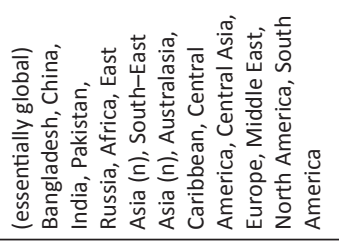 & 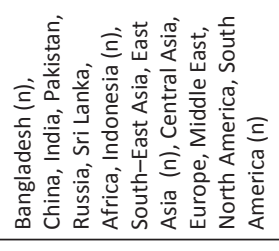 & 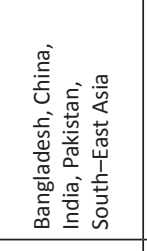 & 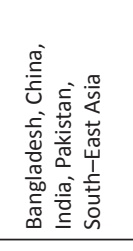 & 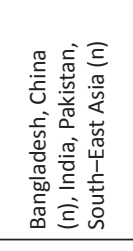 & 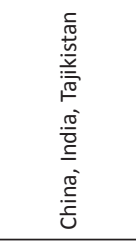 & 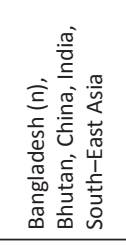 \\
\hline 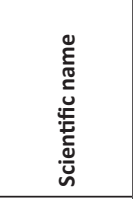 & 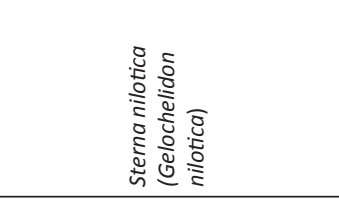 & 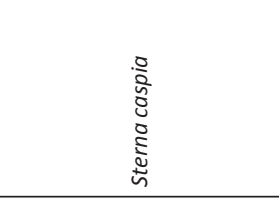 & 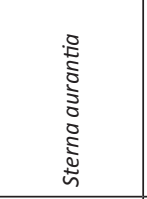 & 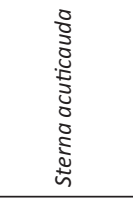 & 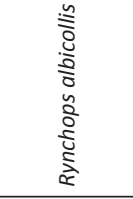 & 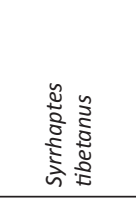 & 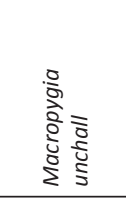 \\
\hline 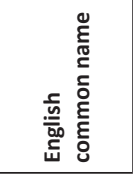 & 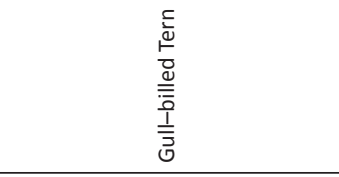 & 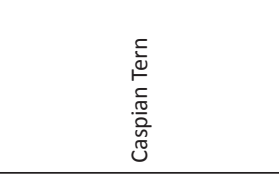 & 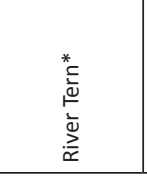 & 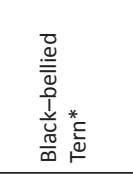 & 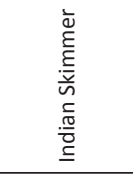 & 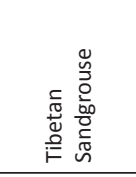 & 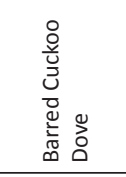 \\
\hline
\end{tabular}




\begin{tabular}{|c|c|c|c|c|c|c|c|c|c|}
\hline 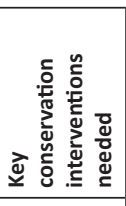 & 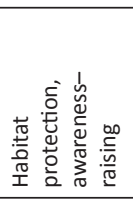 & 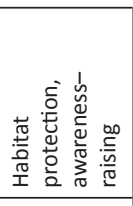 & 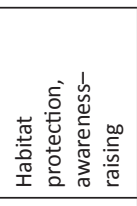 & 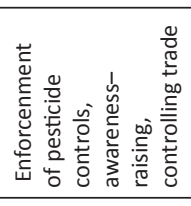 & 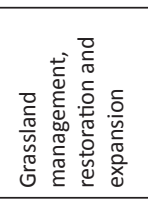 & 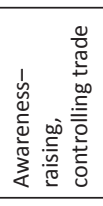 & 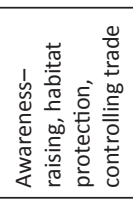 & 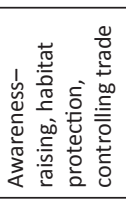 & 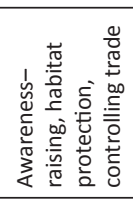 \\
\hline 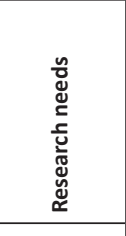 & 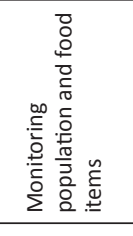 & 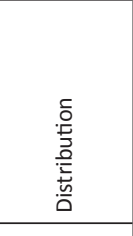 & $\begin{array}{l}\text { 亮 } \\
\text { 产 } \\
\text { 产 } \\
\text { 产 } \\
\end{array}$ & 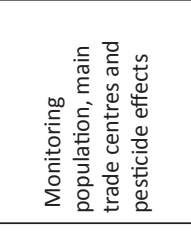 & 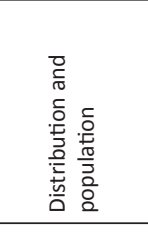 & 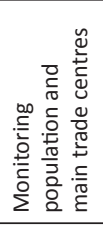 & 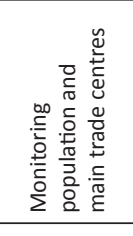 & 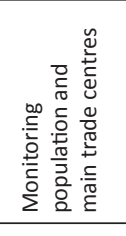 & 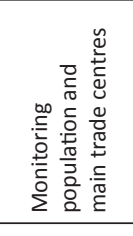 \\
\hline 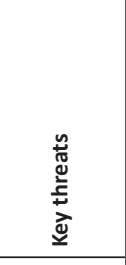 & 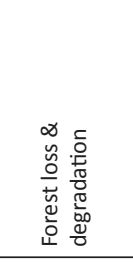 & 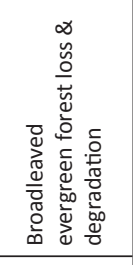 & 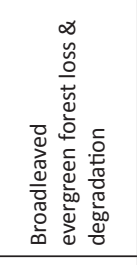 & 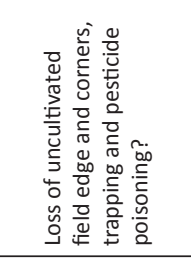 & 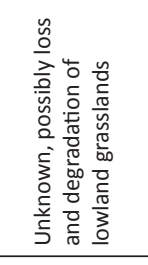 & 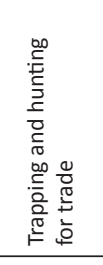 & 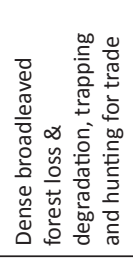 & 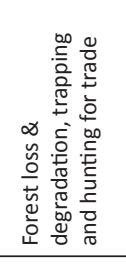 & 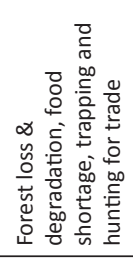 \\
\hline 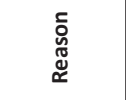 & $\sim$ & 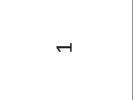 & $r$ & $m$ & $\stackrel{n}{f}$ & $\sim$ & $\stackrel{0}{i}$ & $\stackrel{0}{m}$ & $\sim$ \\
\hline 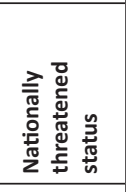 & 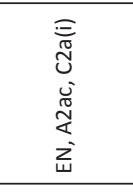 & 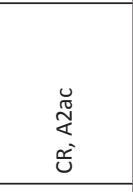 & 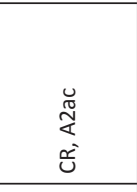 & 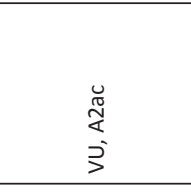 & $\begin{array}{l}\overrightarrow{1} \\
\tilde{O} \\
\tilde{O}\end{array}$ & 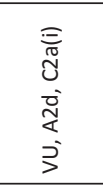 & 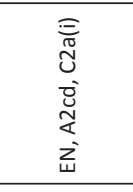 & 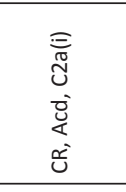 & 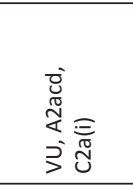 \\
\hline 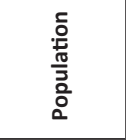 & & & & & 号 & & & & \\
\hline 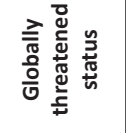 & & & & & & & & & \\
\hline 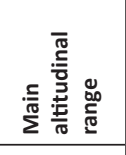 & $\begin{array}{l}\varepsilon \\
0 \\
0 \\
0 \\
n \\
n \\
\end{array}$ & 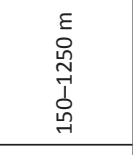 & 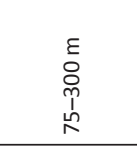 & 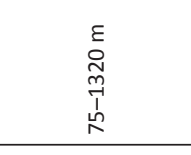 & 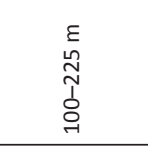 & 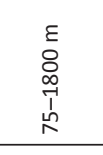 & $\begin{array}{l}\varepsilon \\
0 \\
\stackrel{\leftrightarrow}{N} \\
o \\
\stackrel{\Lambda}{N} \\
\end{array}$ & 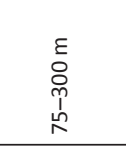 & 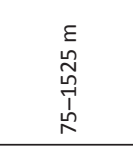 \\
\hline 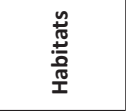 & 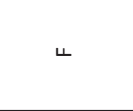 & 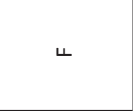 & 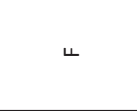 & & ט & ㅇ & 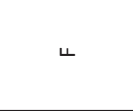 & 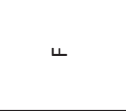 & 3 \\
\hline 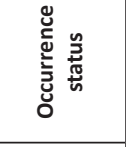 & $\propto$ & $\propto$ & $\propto$ & $\propto$ & $\propto$ & $\propto$ & $\propto$ & $\propto$ & $\propto$ \\
\hline 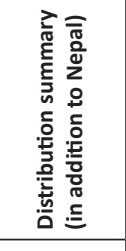 & 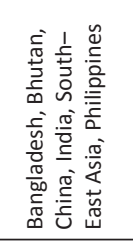 & 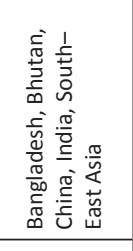 & 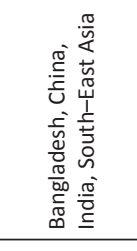 & 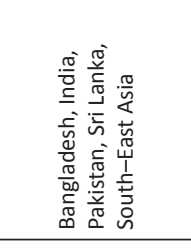 & 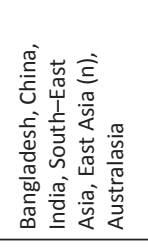 & 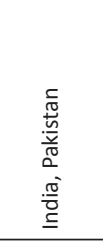 & 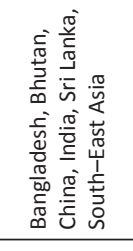 & 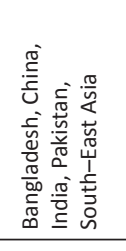 & 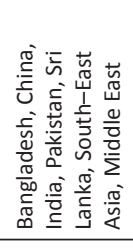 \\
\hline 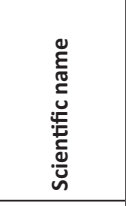 & 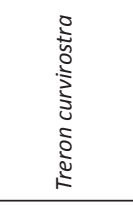 & 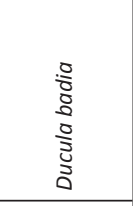 & 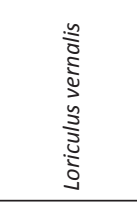 & $\begin{array}{l}0 \\
\frac{0}{0} \\
0 \\
\vdots \\
\end{array}$ & 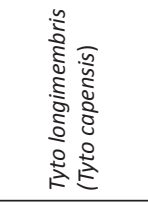 & 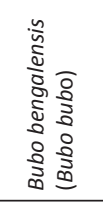 & 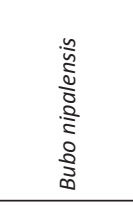 & 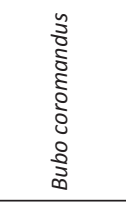 & 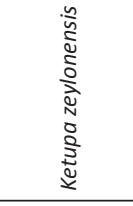 \\
\hline 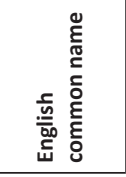 & 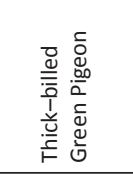 & 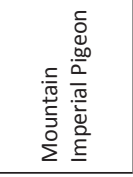 & 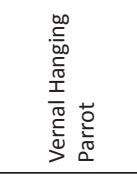 & $\begin{array}{l}\overline{3} \\
0 \\
\frac{5}{5} \\
\infty \\
\end{array}$ & 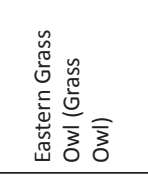 & 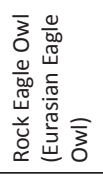 & 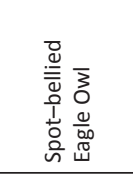 & 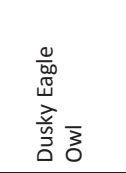 & 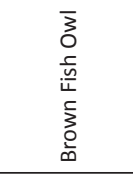 \\
\hline
\end{tabular}




\begin{tabular}{|c|c|c|c|c|c|c|c|c|c|}
\hline 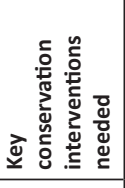 & 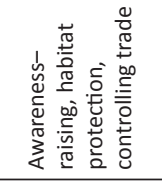 & 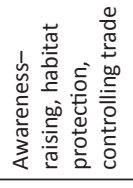 & 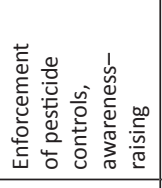 & 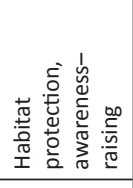 & 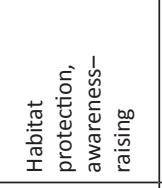 & 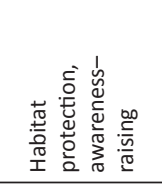 & 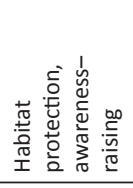 & 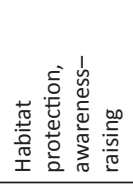 & 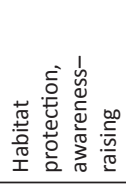 \\
\hline 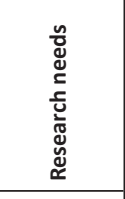 & 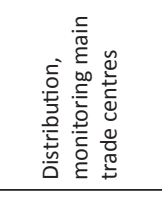 & 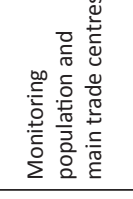 & 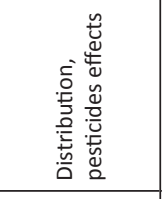 & 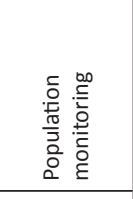 & 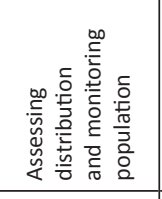 & 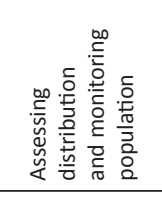 & 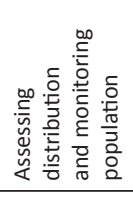 & 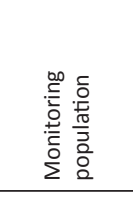 & 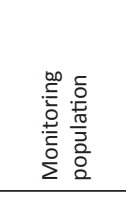 \\
\hline 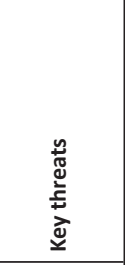 & 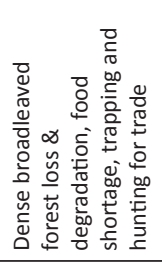 & 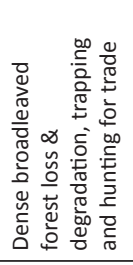 & 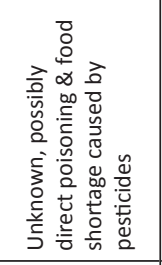 & 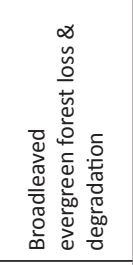 & 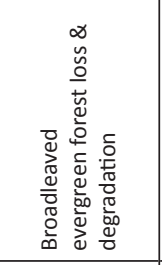 & 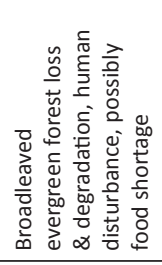 & 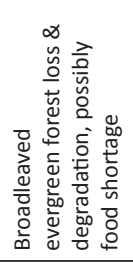 & 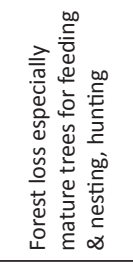 & 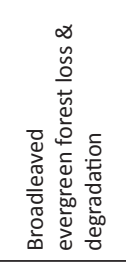 \\
\hline 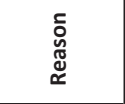 & $\sim$ & $\sim$ & - & $\sim$ & $\stackrel{\bullet}{i}$ & $\sim$ & 6 & $\sim$ & $\sim$ \\
\hline 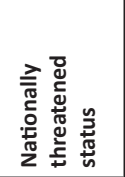 & 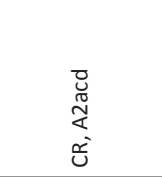 & 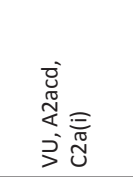 & 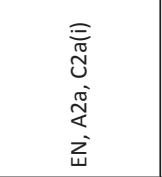 & 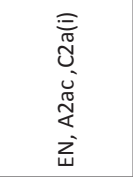 & 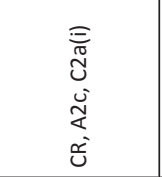 & 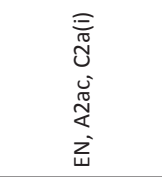 & $\begin{array}{l}\breve{\widetilde{\Psi}} \\
\tilde{U}\end{array}$ & 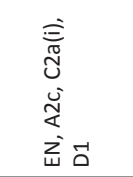 & 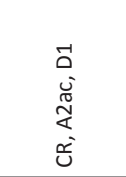 \\
\hline 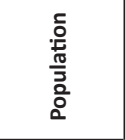 & & & & & & & & $\stackrel{\circ}{\sim}$ & $\stackrel{\rho}{\leftrightarrow}$ \\
\hline 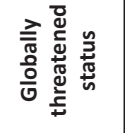 & & & & & & & 上 & z & \\
\hline 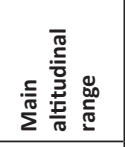 & $\begin{array}{l}E \\
\stackrel{\leftrightarrow}{\circ} \\
m\end{array}$ & $\begin{array}{l}\varepsilon \\
\vdots \\
\vdots \\
\hat{~} \\
\stackrel{\omega}{N} \\
\end{array}$ & 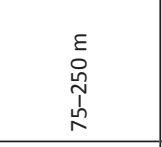 & 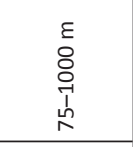 & $\begin{array}{l}\varepsilon \\
0 \\
0 \\
0 \\
0 \\
\end{array}$ & 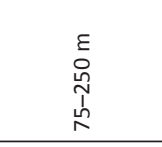 & $\begin{array}{l}E \\
\text { E } \\
N\end{array}$ & 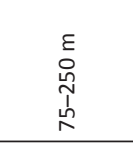 & 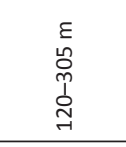 \\
\hline 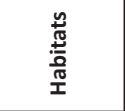 & 3 & 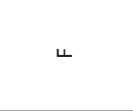 & $\tilde{y}$ & 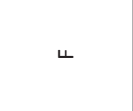 & 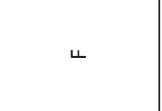 & 3 & ч & 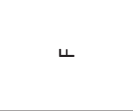 & ¿ \\
\hline 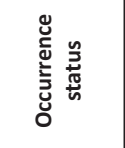 & $\propto$ & $\propto$ & $\propto$ & $\propto$ & $\check{\simeq}$ & $\propto$ & $\propto$ & $\propto$ & $\propto$ \\
\hline 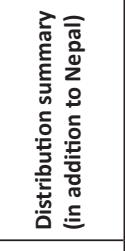 & 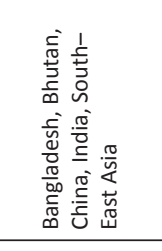 & 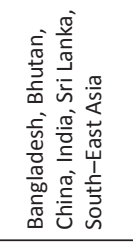 & 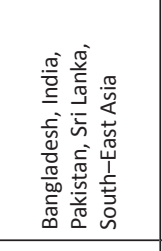 & 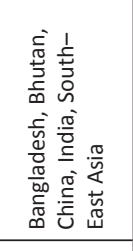 & 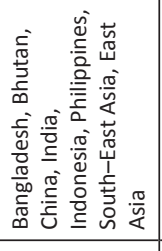 & 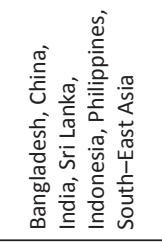 & 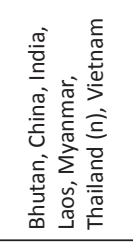 & 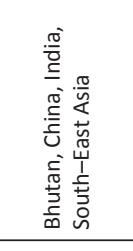 & 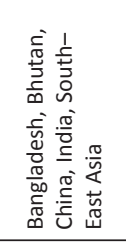 \\
\hline 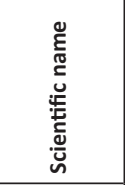 & 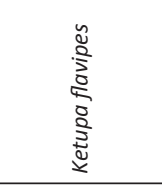 & 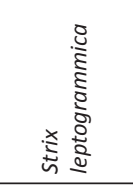 & 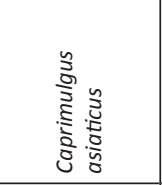 & 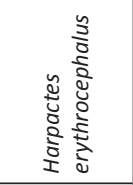 & 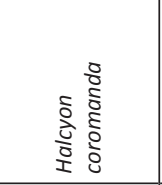 & 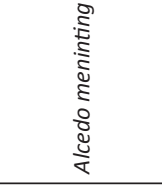 & 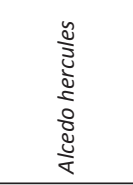 & 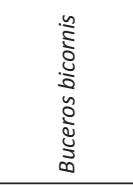 & 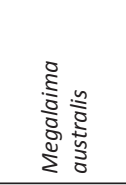 \\
\hline 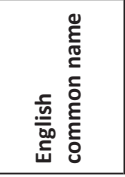 & 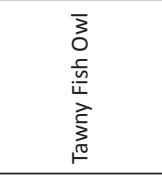 & 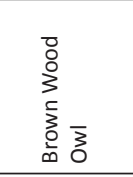 & 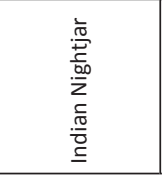 & 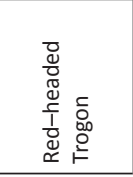 & 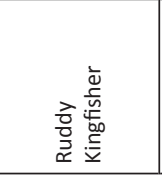 & 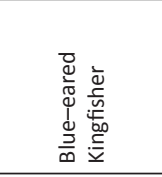 & 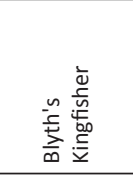 & 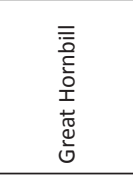 & 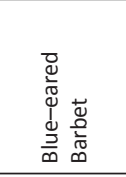 \\
\hline
\end{tabular}




\begin{tabular}{|c|c|c|c|c|c|c|c|c|c|}
\hline 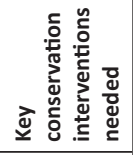 & 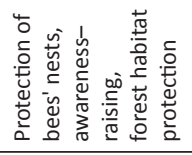 & 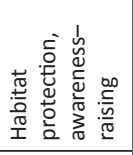 & 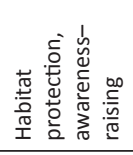 & 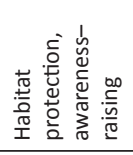 & 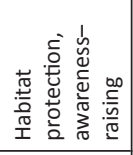 & 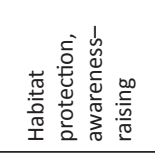 & 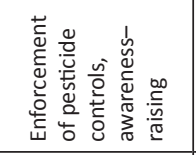 & 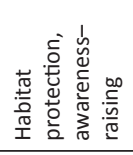 & 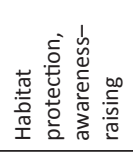 \\
\hline 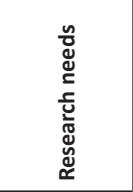 & 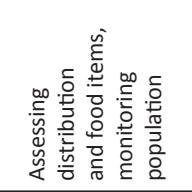 & 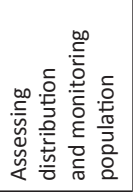 & 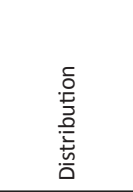 & 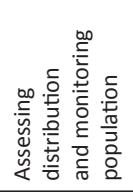 & 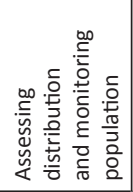 & 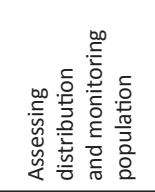 & 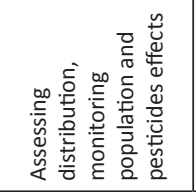 & 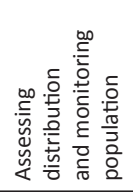 & 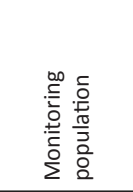 \\
\hline 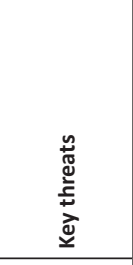 & 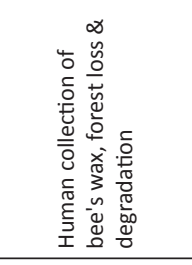 & 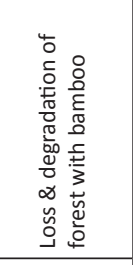 & 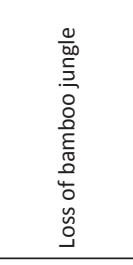 & 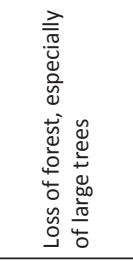 & 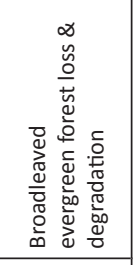 & 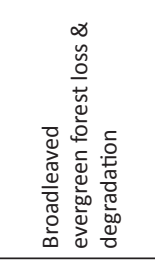 & 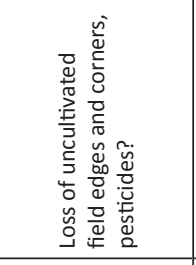 & 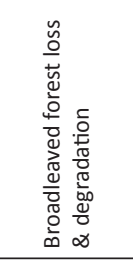 & 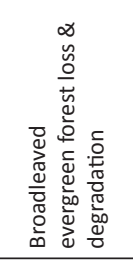 \\
\hline 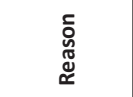 & $\sim$ & $\sim$ & $r$ & $\sim$ & $\sim$ & $\sim$ & $\sim$ & 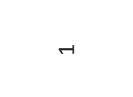 & $\sim$ \\
\hline 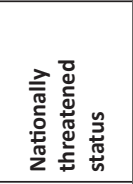 & 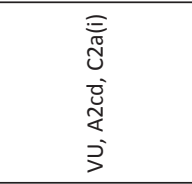 & 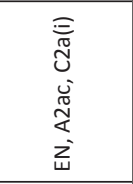 & 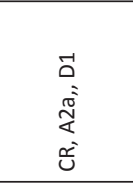 & 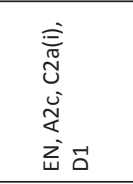 & $\begin{array}{l}\tilde{\widetilde{T}} \\
\text { 㟧 }\end{array}$ & 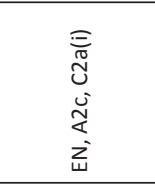 & 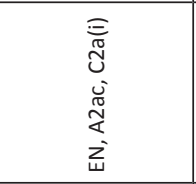 & 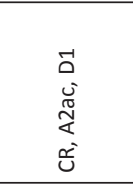 & 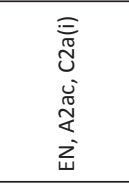 \\
\hline 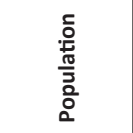 & & & 号 & 品 & & & & 号 & \\
\hline 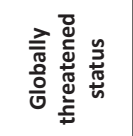 & z & & & 3 & & & & & \\
\hline 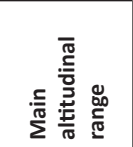 & 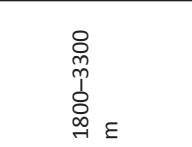 & 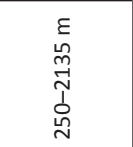 & $\underset{\substack{E \\
N}}{ }$ & 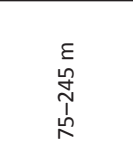 & $\begin{array}{l}E \\
\stackrel{\sim}{N} \\
\stackrel{ِ}{A}\end{array}$ & 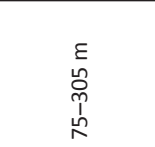 & 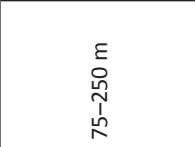 & 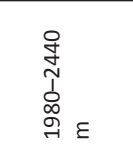 & 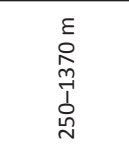 \\
\hline 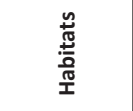 & u & 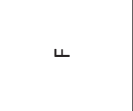 & 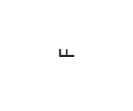 & ч & 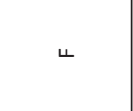 & 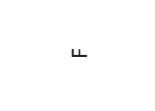 & u & 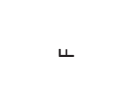 & 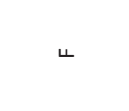 \\
\hline 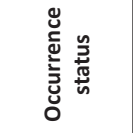 & $\propto$ & $\propto$ & $\propto$ & $\propto$ & $\widetilde{\propto}$ & तs & $\propto$ & $\propto$ & $\propto$ \\
\hline 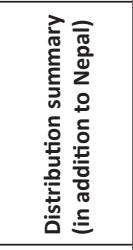 & 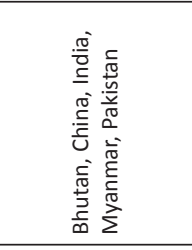 & 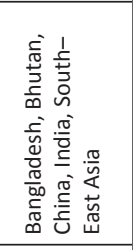 & 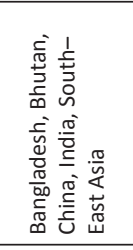 & 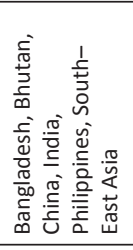 & 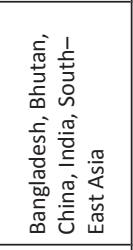 & 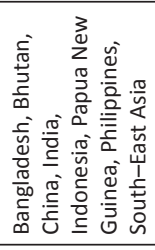 & 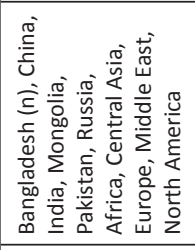 & 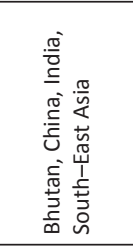 & 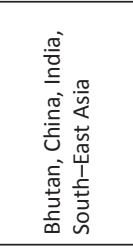 \\
\hline 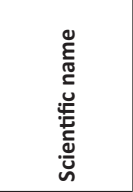 & 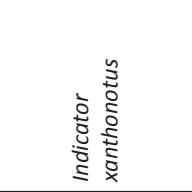 & 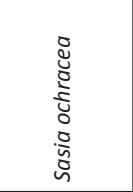 & 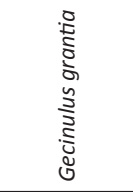 & 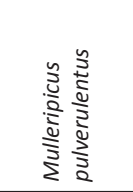 & 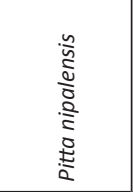 & 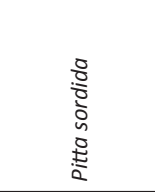 & 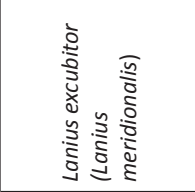 & 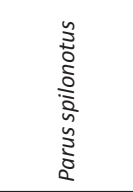 & 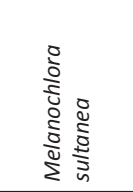 \\
\hline 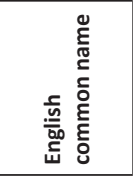 & 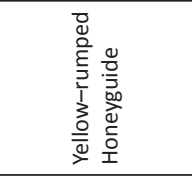 & 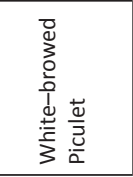 & 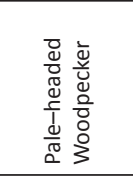 & 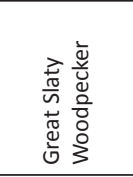 & 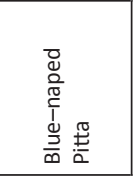 & 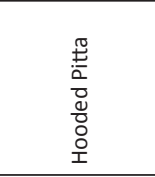 & 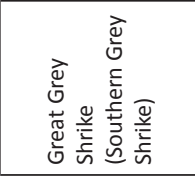 & 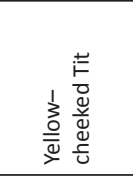 & $\begin{array}{l}\vec{*} \\
\stackrel{5}{c} \\
\frac{c}{w} \\
\bar{n}\end{array}$ \\
\hline
\end{tabular}




\begin{tabular}{|c|c|c|c|c|c|c|c|c|c|c|}
\hline 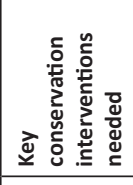 & 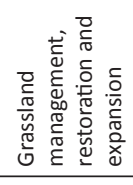 & 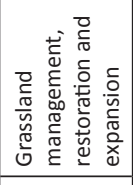 & 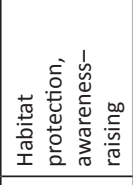 & 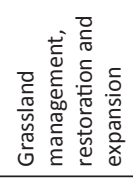 & 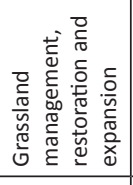 & 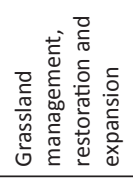 & 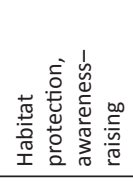 & 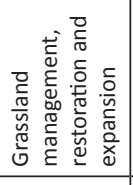 & 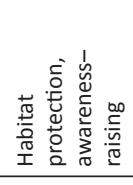 & 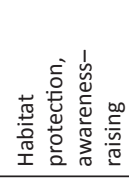 \\
\hline 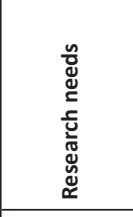 & 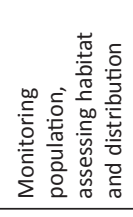 & 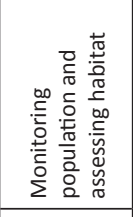 & 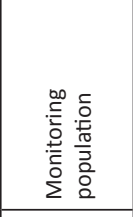 & 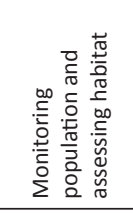 & 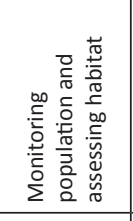 & 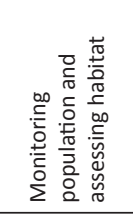 & 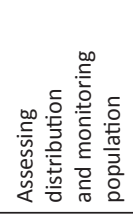 & 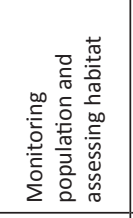 & 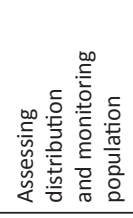 & 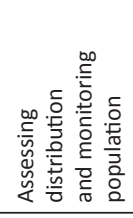 \\
\hline 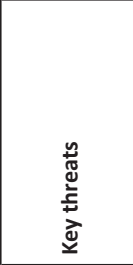 & 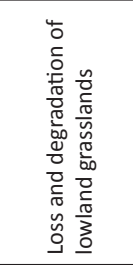 & 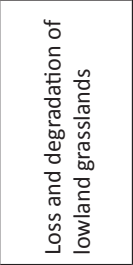 & 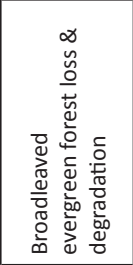 & 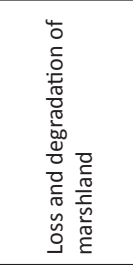 & 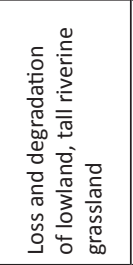 & 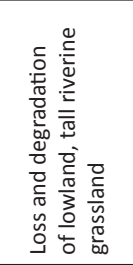 & 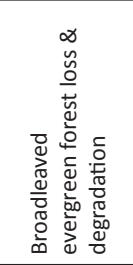 & 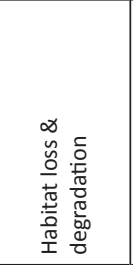 & 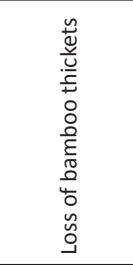 & 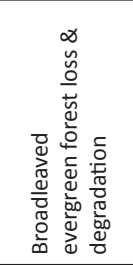 \\
\hline 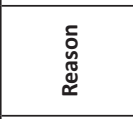 & $\begin{array}{l}0 \\
0 \\
N \\
i \\
+1\end{array}$ & $\begin{array}{l}n \\
\stackrel{\leftrightarrow}{+} \\
r\end{array}$ & $\sim$ & $\begin{array}{l}0 \\
i\end{array}$ & in & $\sigma$ & N & $\sigma$ & $\sim$ & $\sim$ \\
\hline 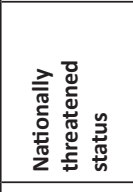 & 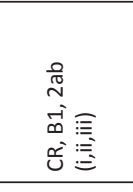 & 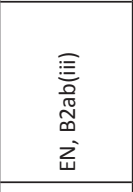 & 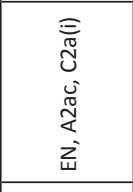 & $\begin{array}{l}\underset{\widetilde{T}}{\pi} \\
\tilde{\tilde{C}}\end{array}$ & 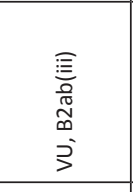 & $\begin{array}{l}\bar{\equiv} \\
\text { 言 } \\
\infty \\
\infty \\
\text { ż } \\
\end{array}$ & 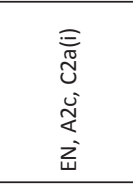 & 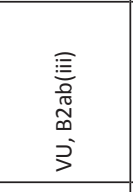 & 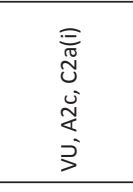 & 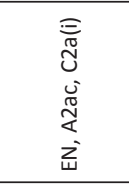 \\
\hline 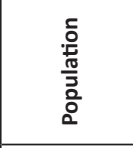 & $\underset{\stackrel{O}{v}}{\text { v }}$ & 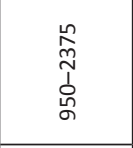 & & 品 & 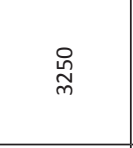 & 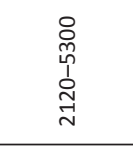 & & 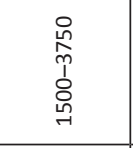 & & \\
\hline 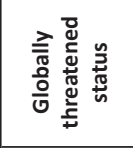 & z & 3 & & & 3 & z & & & & \\
\hline 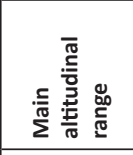 & 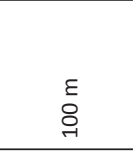 & $\begin{array}{l}E \\
ٌ \\
0 \\
0 \\
1 \\
\end{array}$ & 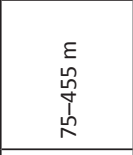 & 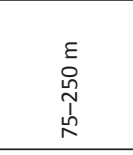 & 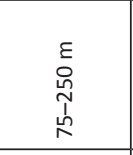 & 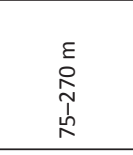 & $\begin{array}{l}8 \\
\stackrel{1}{1} \\
1 \\
0 \\
0 \\
-1 \\
\end{array}$ & 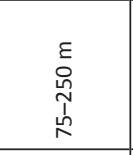 & 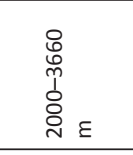 & 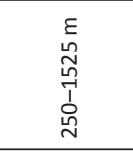 \\
\hline 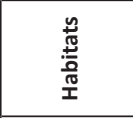 & ৩ & 0 & 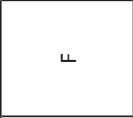 & ט & 0 & ৩ & 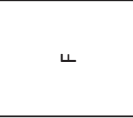 & $\tilde{\nu}$ & ч & 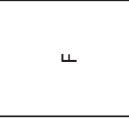 \\
\hline 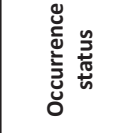 & $\propto$ & $\propto$ & $\propto$ & $\propto$ & $\widetilde{\propto}$ & $\propto$ & $\propto$ & $\propto$ & $\propto$ & $\approx$ \\
\hline 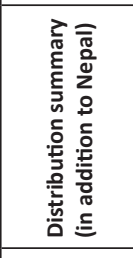 & 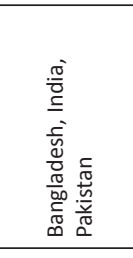 & 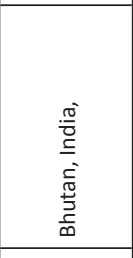 & 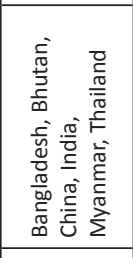 & 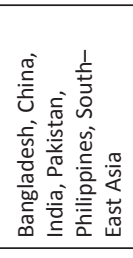 & 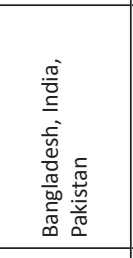 & 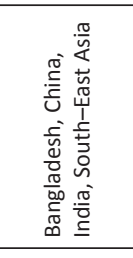 & 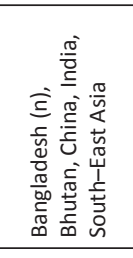 & 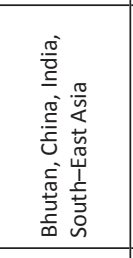 & 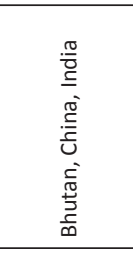 & 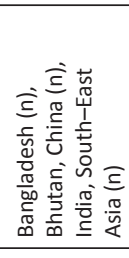 \\
\hline 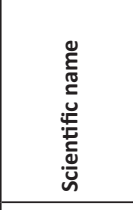 & 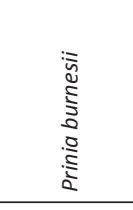 & 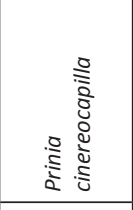 & 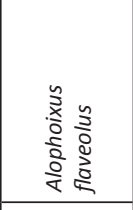 & 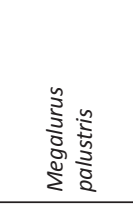 & 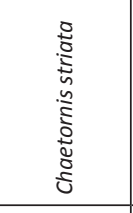 & 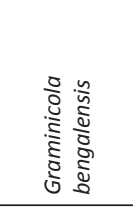 & 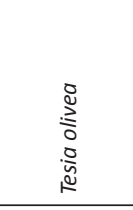 & 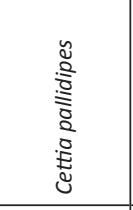 & 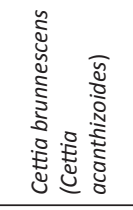 & 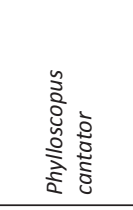 \\
\hline 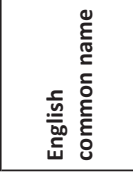 & 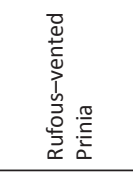 & 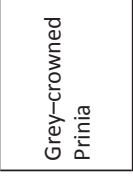 & 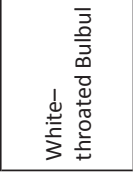 & 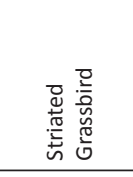 & 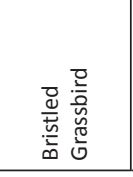 & 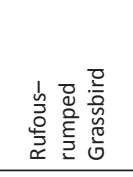 & 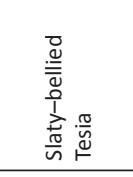 & 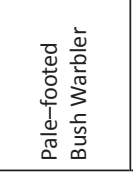 & 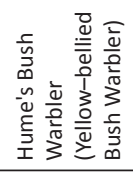 & 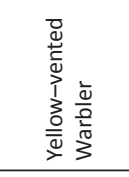 \\
\hline
\end{tabular}




\begin{tabular}{|c|c|c|c|c|c|c|c|c|c|c|}
\hline 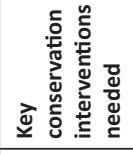 & 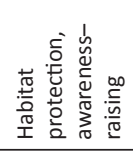 & 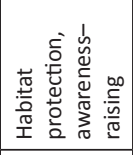 & 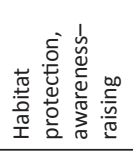 & 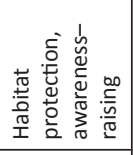 & 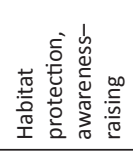 & 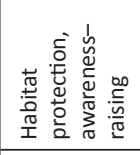 & 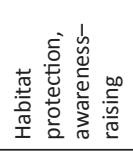 & 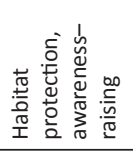 & 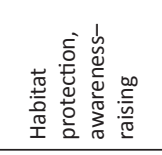 & 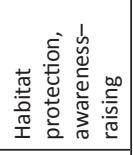 \\
\hline 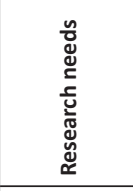 & 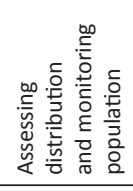 & 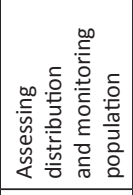 & 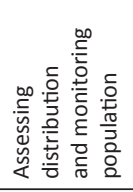 & 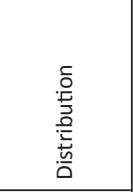 & 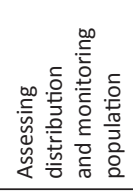 & 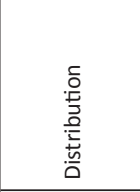 & 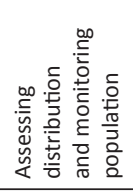 & 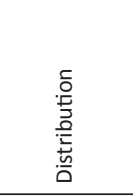 & 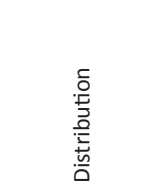 & 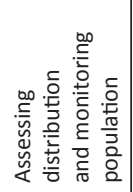 \\
\hline 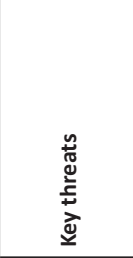 & 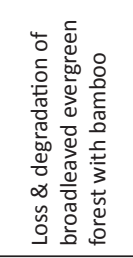 & 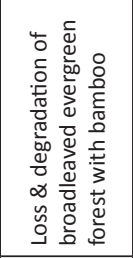 & 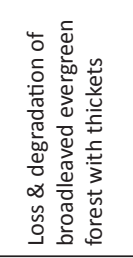 & 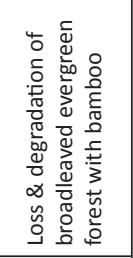 & 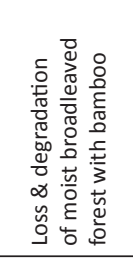 & 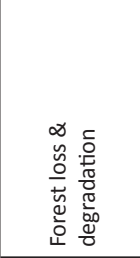 & 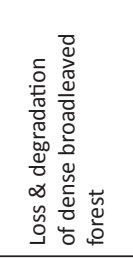 & 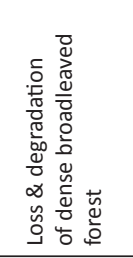 & 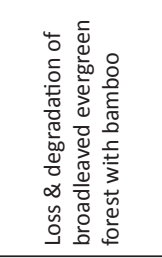 & 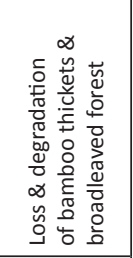 \\
\hline 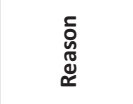 & $\sim$ & 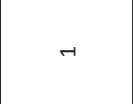 & $\sim$ & $r$ & $\sim$ & 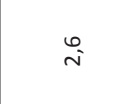 & $\sim$ & $\stackrel{i}{i}$ & $\stackrel{0}{i}$ & N \\
\hline 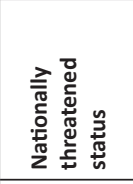 & 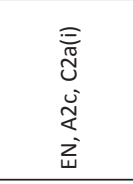 & 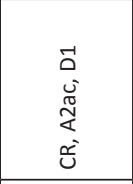 & 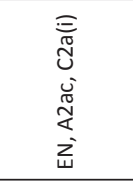 & 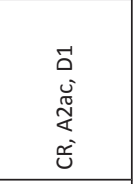 & 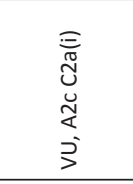 & $\begin{array}{l}\breve{\widetilde{T}} \\
\tilde{\widetilde{I}} \\
\end{array}$ & 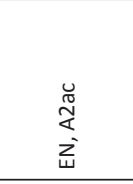 & $\begin{array}{l}\breve{\Psi} \\
\tilde{\Psi} \\
\tilde{\tilde{U}}\end{array}$ & 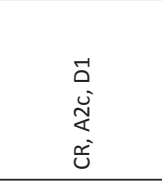 & 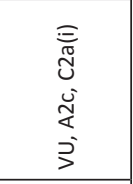 \\
\hline 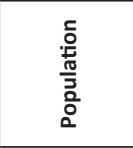 & & $\stackrel{\leftrightarrow}{\Omega}$ & & $\stackrel{\leftrightarrow}{\vartheta}$ & & & & & $\stackrel{\circ}{\vartheta}$ & \\
\hline 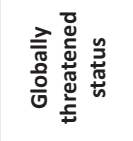 & & & & & & & 5 & & 占 & \\
\hline 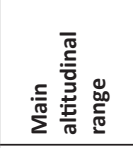 & 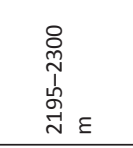 & 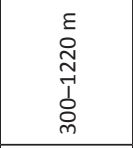 & 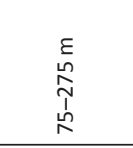 & 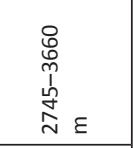 & 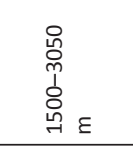 & 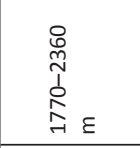 & 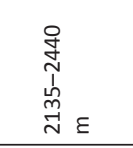 & 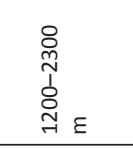 & 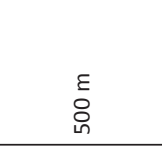 & 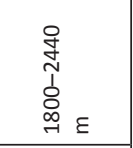 \\
\hline 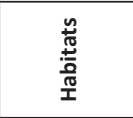 & 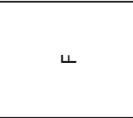 & ч & ч & 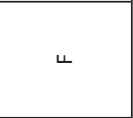 & 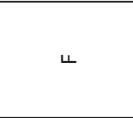 & 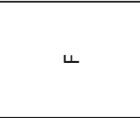 & 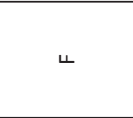 & 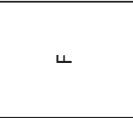 & ч & 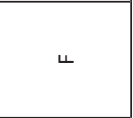 \\
\hline 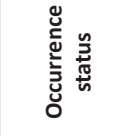 & $\propto$ & $\propto$ & $\propto$ & $\propto$ & $\propto$ & $\propto$ & $\propto$ & $\propto$ & $\propto$ & $\propto$ \\
\hline 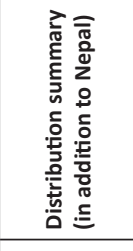 & 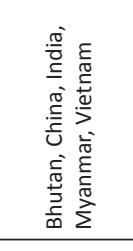 & 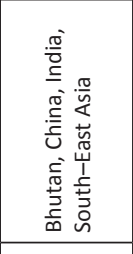 & 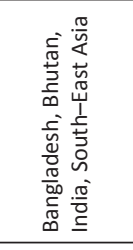 & 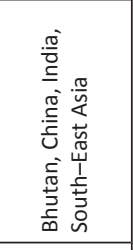 & 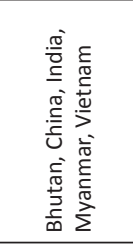 & 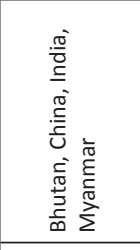 & 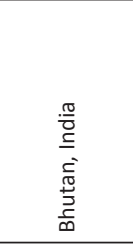 & 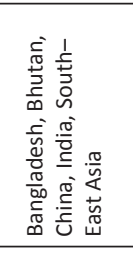 & 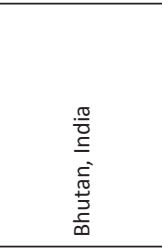 & 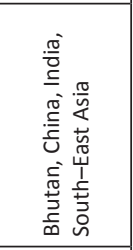 \\
\hline 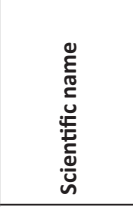 & 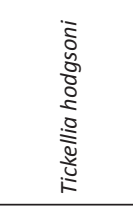 & 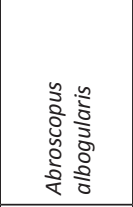 & 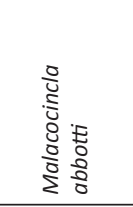 & 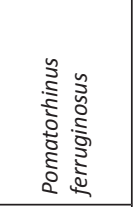 & 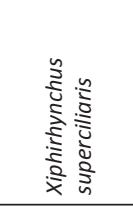 & 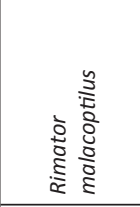 & 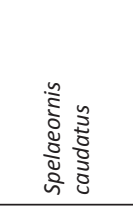 & 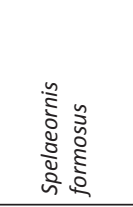 & 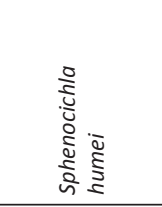 & 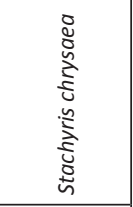 \\
\hline 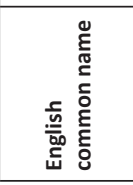 & 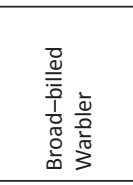 & 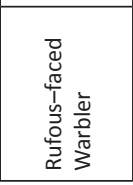 & 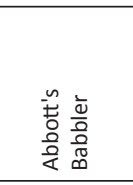 & 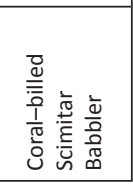 & 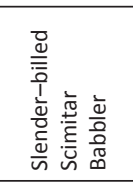 & 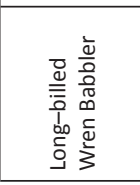 & 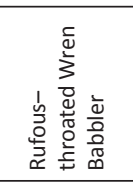 & 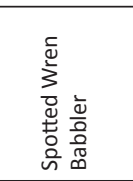 & 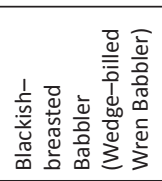 & 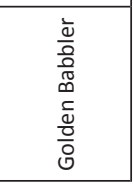 \\
\hline
\end{tabular}




\begin{tabular}{|c|c|c|c|c|c|c|c|c|c|c|c|}
\hline 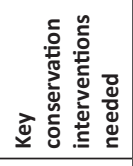 & 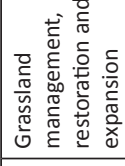 & 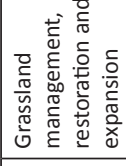 & 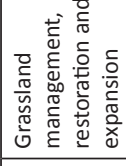 & 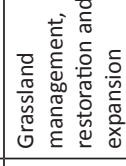 & 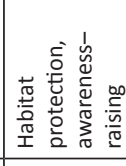 & 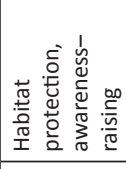 & 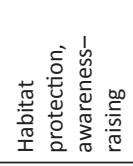 & 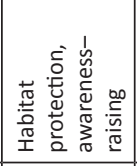 & 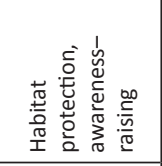 & 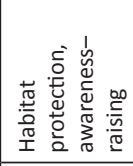 & 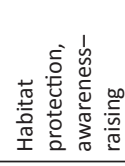 \\
\hline 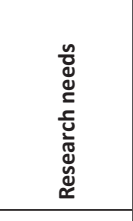 & 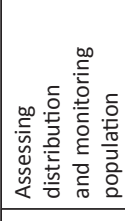 & 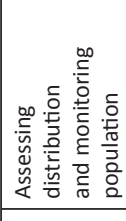 & 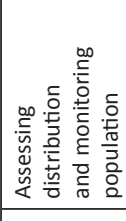 & 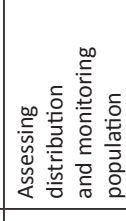 & 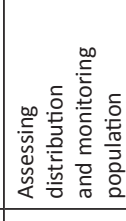 & 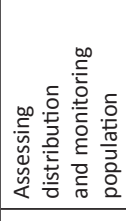 & 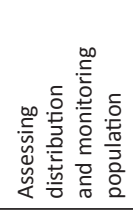 & 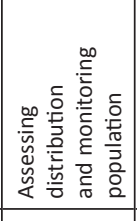 & 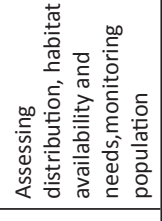 & 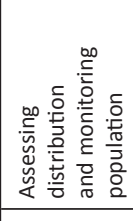 & 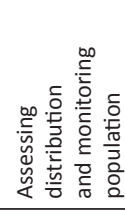 \\
\hline 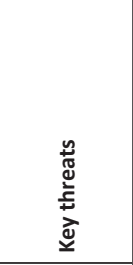 & 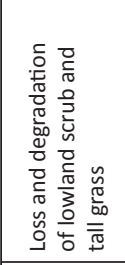 & 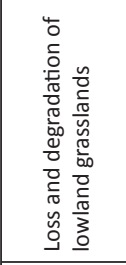 & 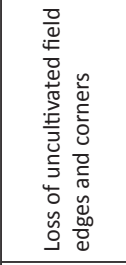 & 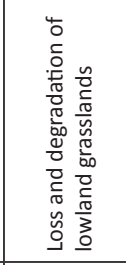 & 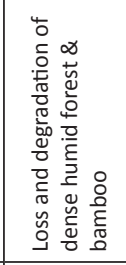 & 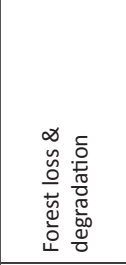 & 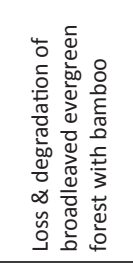 & 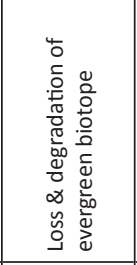 & 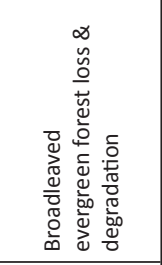 & 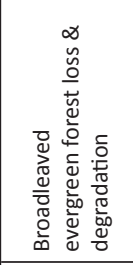 & 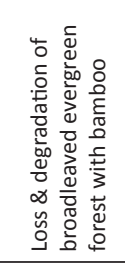 \\
\hline 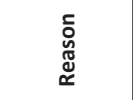 & $\sim$ & 0 & $\sigma$ & $\begin{array}{l}0 \\
\sim\end{array}$ & $\sim$ & $\sim$ & $\sim$ & H & $\sim$ & $\sim$ & 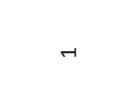 \\
\hline 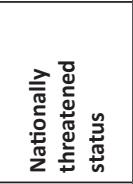 & 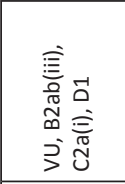 & 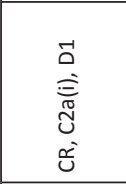 & 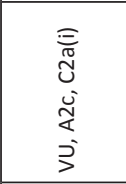 & 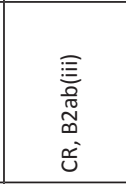 & 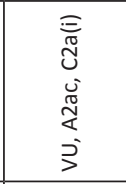 & 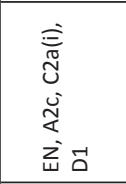 & 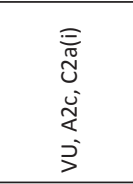 & 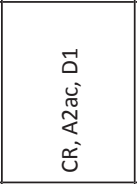 & 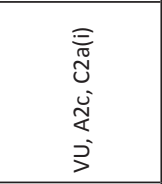 & 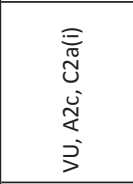 & 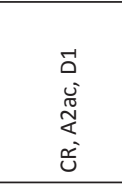 \\
\hline 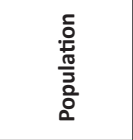 & : & 号 & & $\begin{array}{l}0 \\
0 \\
\stackrel{1}{1} \\
\stackrel{N}{N}\end{array}$ & & 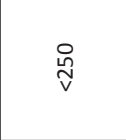 & & $\stackrel{\leftrightarrow}{\vartheta}$ & & & 号 \\
\hline 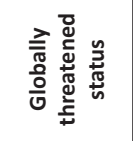 & & 3 & & 3 & & & & & & & \\
\hline 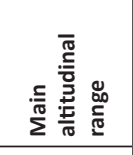 & 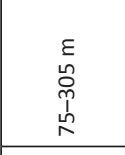 & 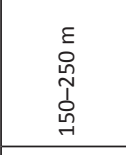 & 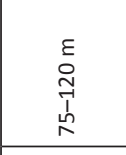 & $\begin{array}{l}\varepsilon \\
\text { d. } \\
\end{array}$ & 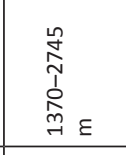 & 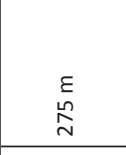 & 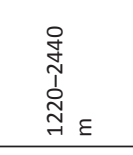 & 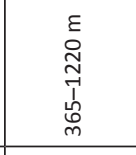 & 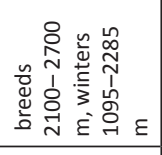 & 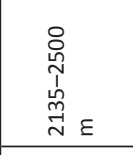 & $\begin{array}{l}\varepsilon \\
8 \\
0 \\
\vdots \\
8 \\
8 \\
0\end{array}$ \\
\hline 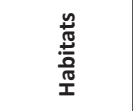 & $\widehat{n}$ & ט & $\tilde{y}$ & ৩ & 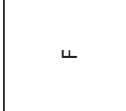 & ч & 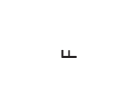 & u & 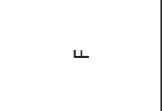 & ч & $u$ \\
\hline 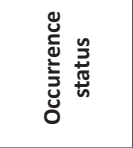 & $\propto$ & $\propto$ & $\propto$ & $\propto$ & $\propto$ & $\propto$ & $\propto$ & $\propto$ & $\propto$ & $\propto$ & $\propto$ \\
\hline 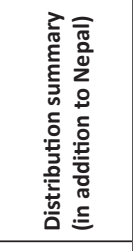 & 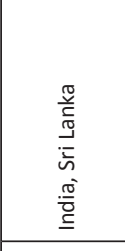 & 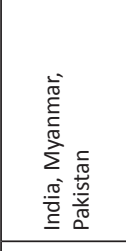 & 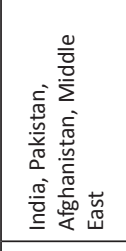 & 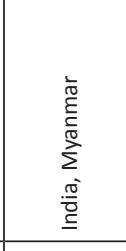 & 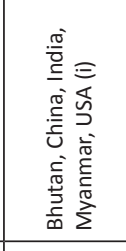 & 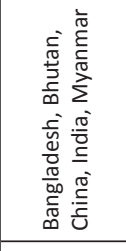 & 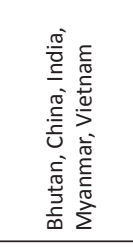 & 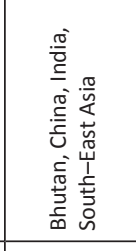 & 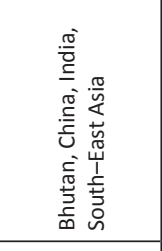 & 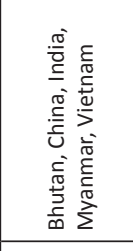 & 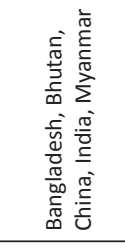 \\
\hline 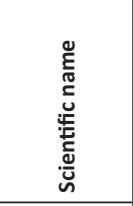 & 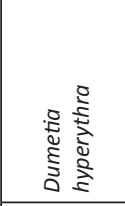 & 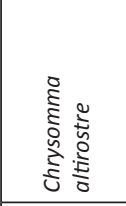 & 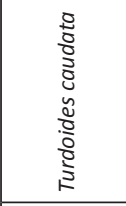 & 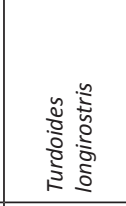 & 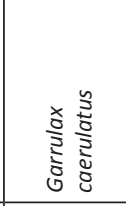 & 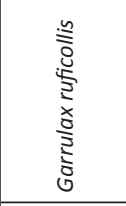 & 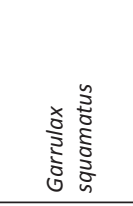 & 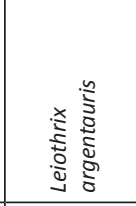 & 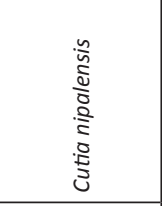 & 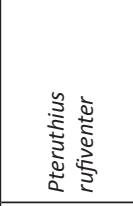 & 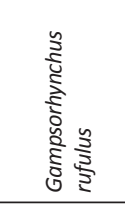 \\
\hline 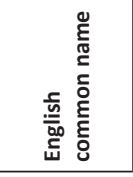 & 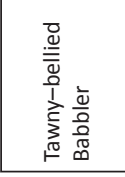 & 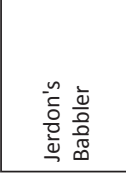 & 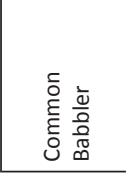 & 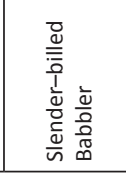 & 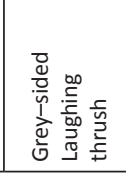 & 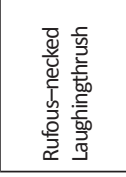 & 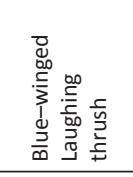 & 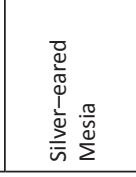 & 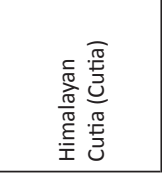 & 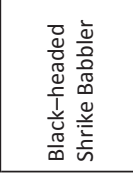 & 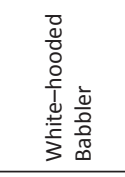 \\
\hline
\end{tabular}




\begin{tabular}{|c|c|c|c|c|c|c|c|c|c|c|}
\hline 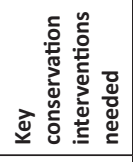 & 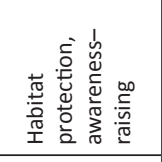 & 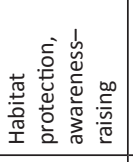 & 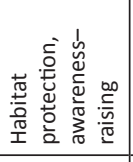 & 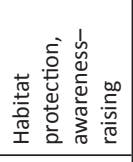 & 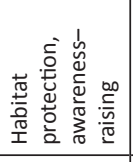 & 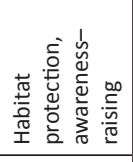 & 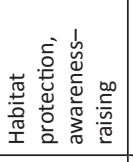 & 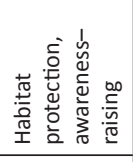 & 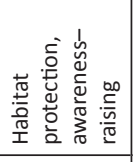 & 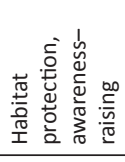 \\
\hline 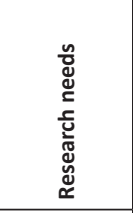 & 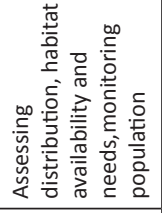 & 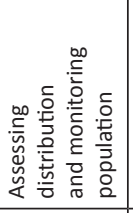 & 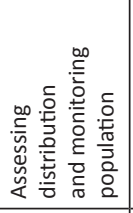 & 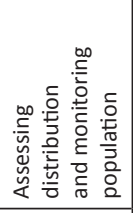 & 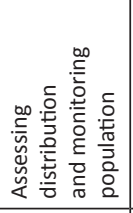 & 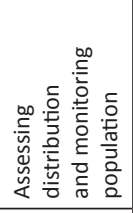 & 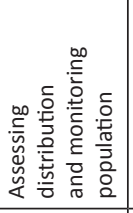 & 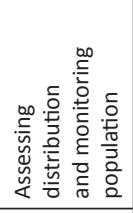 & 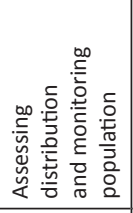 & 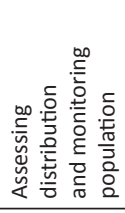 \\
\hline 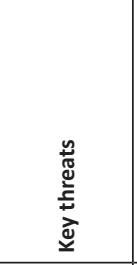 & 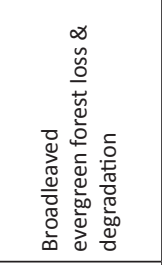 & 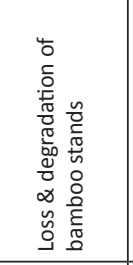 & 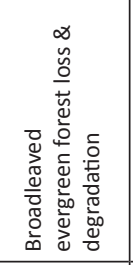 & 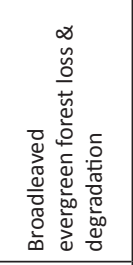 & 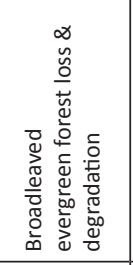 & 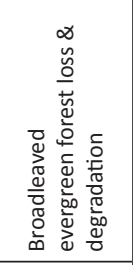 & 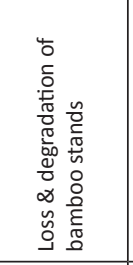 & 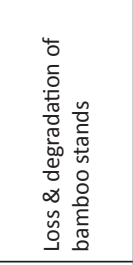 & 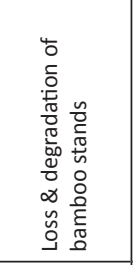 & 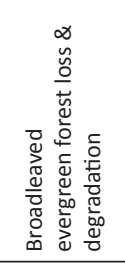 \\
\hline 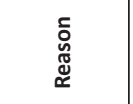 & $\sim$ & $\sim$ & - & $\rightarrow$ & $\sim$ & $\sim$ & $\sim$ & $\sim$ & $\sim$ & $r$ \\
\hline 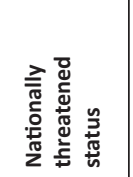 & 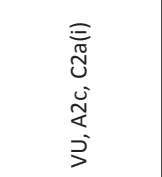 & 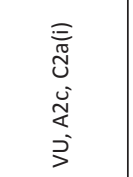 & 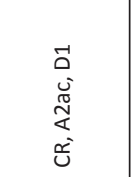 & 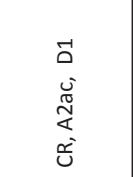 & 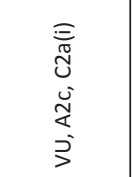 & 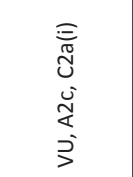 & 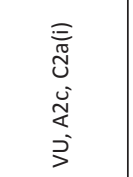 & 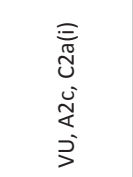 & 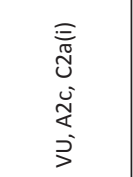 & 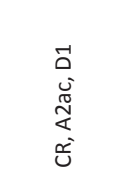 \\
\hline 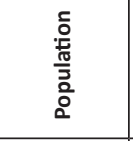 & & & คे & 号 & & & & & & คे \\
\hline 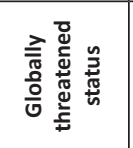 & & & & & & & & & & \\
\hline 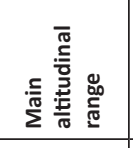 & 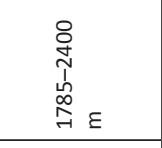 & 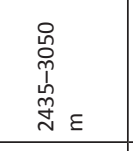 & 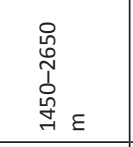 & 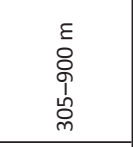 & 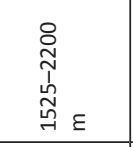 & $\begin{array}{l}\varepsilon \\
0 \\
0 \\
1 \\
1 \\
0 \\
d\end{array}$ & 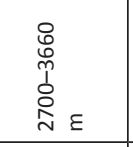 & 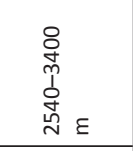 & 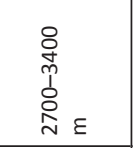 & 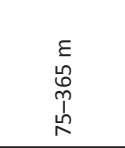 \\
\hline 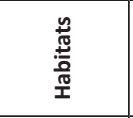 & 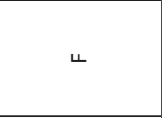 & 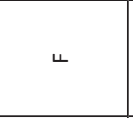 & 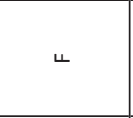 & 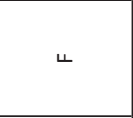 & 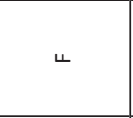 & 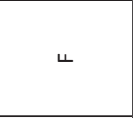 & 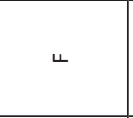 & 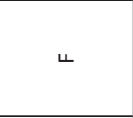 & 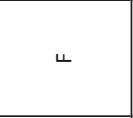 & 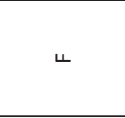 \\
\hline 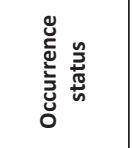 & $\propto$ & $\propto$ & $\propto$ & $\propto$ & $\propto$ & $\propto$ & $\propto$ & $\propto$ & $\propto$ & $\propto$ \\
\hline 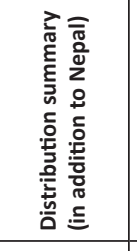 & 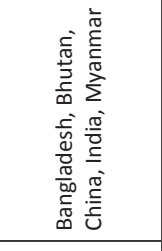 & 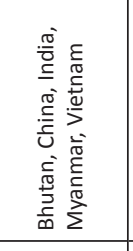 & 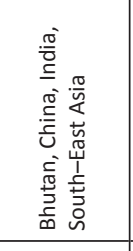 & 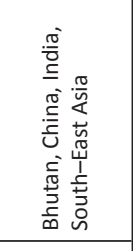 & 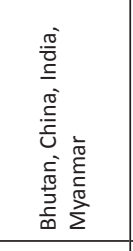 & 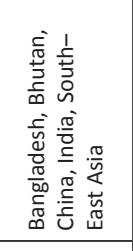 & 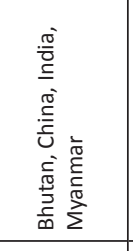 & 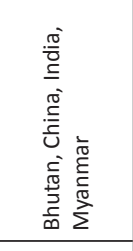 & 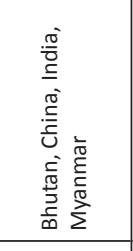 & 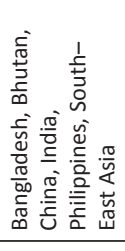 \\
\hline 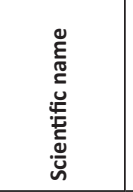 & 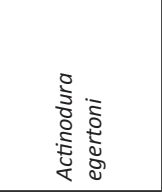 & 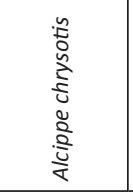 & 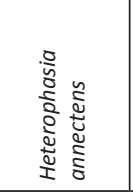 & 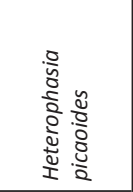 & 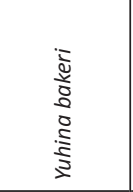 & 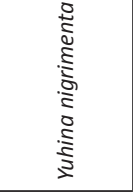 & 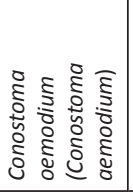 & 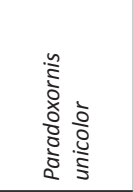 & 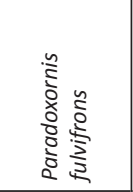 & 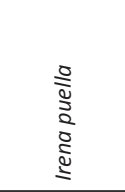 \\
\hline 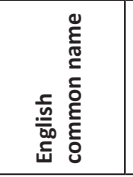 & 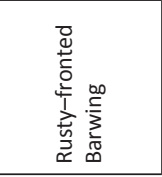 & 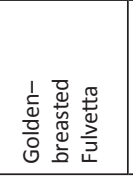 & 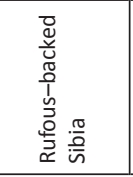 & 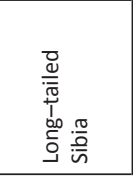 & 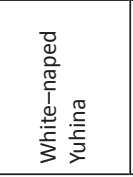 & 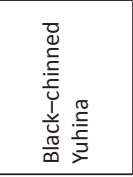 & 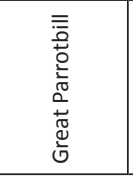 & 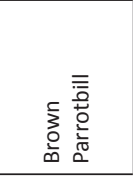 & 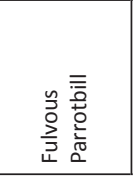 & 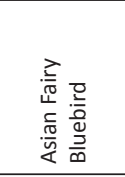 \\
\hline
\end{tabular}




\begin{tabular}{|c|c|c|c|c|c|c|c|c|c|c|}
\hline 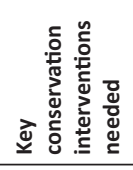 & 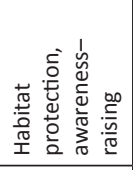 & 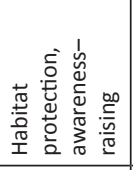 & 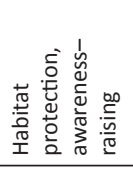 & 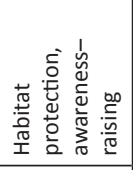 & 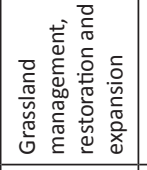 & 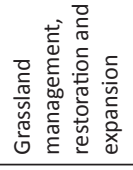 & 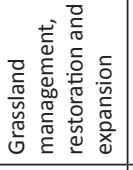 & 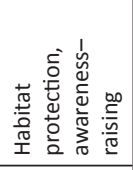 & 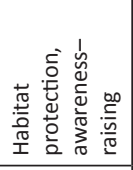 & \\
\hline 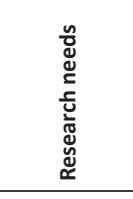 & 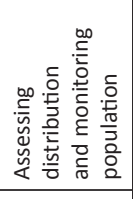 & 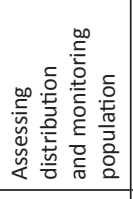 & 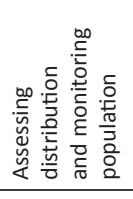 & 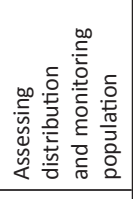 & 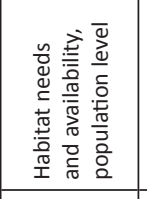 & 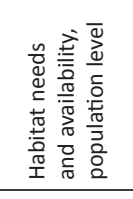 & 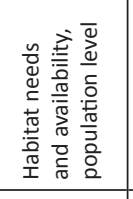 & 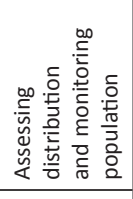 & 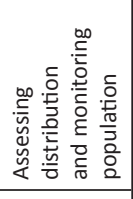 & 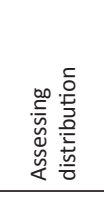 \\
\hline 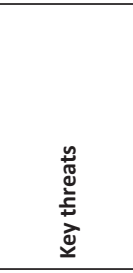 & 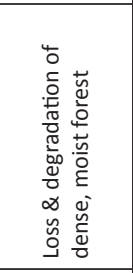 & 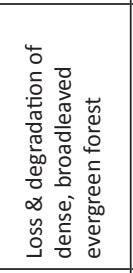 & 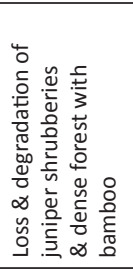 & 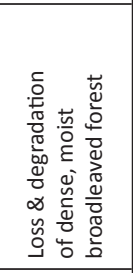 & 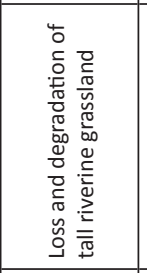 & 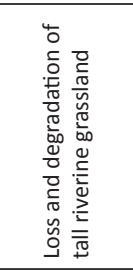 & 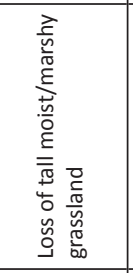 & 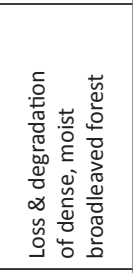 & 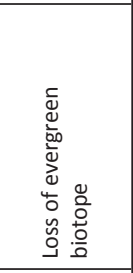 & 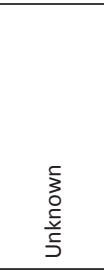 \\
\hline 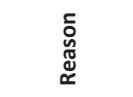 & N & $\sim$ & $\sim$ & - & $\begin{array}{l}0 \\
i\end{array}$ & \begin{tabular}{l}
0 \\
\multirow{f}{*}{}
\end{tabular} & in & $\sim$ & - & - \\
\hline 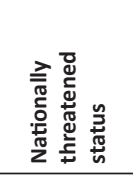 & 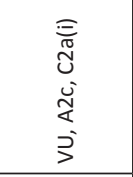 & 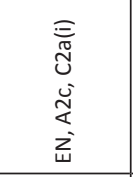 & 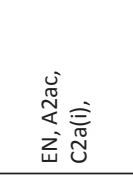 & 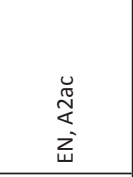 & 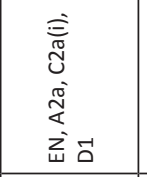 & 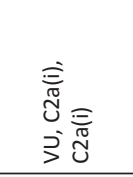 & 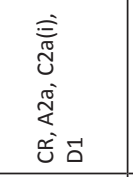 & 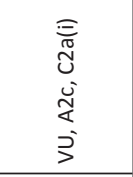 & 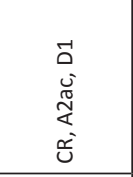 & 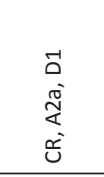 \\
\hline 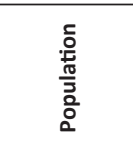 & & & & & $\stackrel{\stackrel{\leftrightarrow}{v}}{v}$ & & $\stackrel{\leftrightarrow}{\vartheta}$ & & 号 & 误 \\
\hline 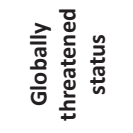 & & & & & ૩ & & & & & \\
\hline 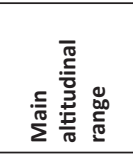 & 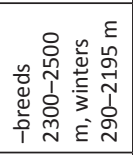 & $\begin{array}{l}\varepsilon \\
o \\
o \\
\tilde{p} \\
\vdots \\
ٌ \\
\sigma\end{array}$ & 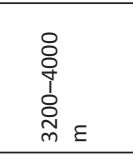 & 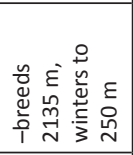 & $\begin{array}{l}\varepsilon \\
\vdots \\
\stackrel{\Lambda}{N} \\
\Lambda \\
\Lambda\end{array}$ & 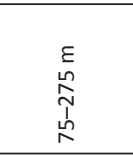 & 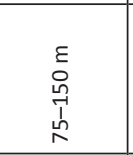 & $\begin{array}{l}\varepsilon \\
o \\
o \\
\tilde{m} \\
\omega \\
\omega\end{array}$ & 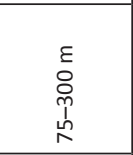 & 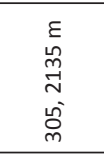 \\
\hline 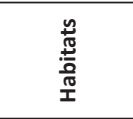 & 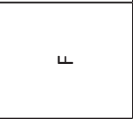 & 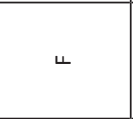 & ч & 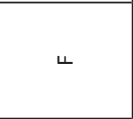 & o & ט & ט & 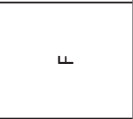 & ч & 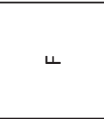 \\
\hline 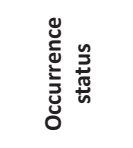 & $\approx$ & $\propto$ & $\propto$ & $\propto$ & $\gtreqless$ & $\propto$ & $\propto$ & $\propto$ & $\propto$ & $\approx$ \\
\hline 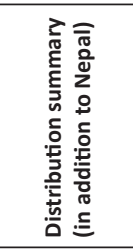 & 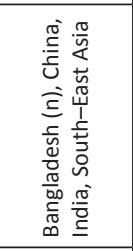 & 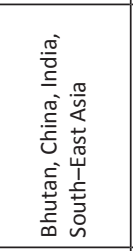 & 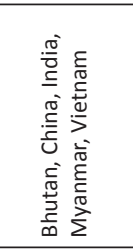 & 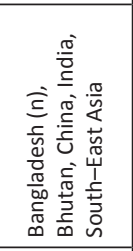 & 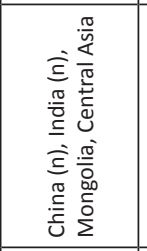 & 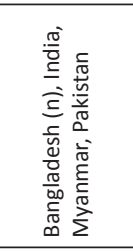 & 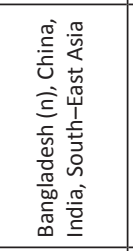 & 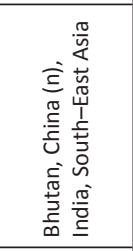 & 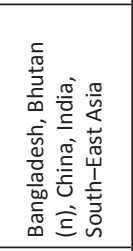 & 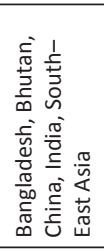 \\
\hline 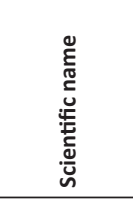 & 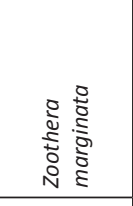 & 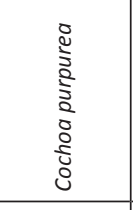 & 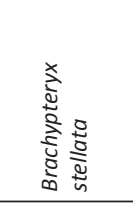 & 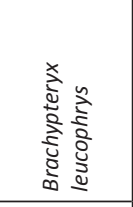 & 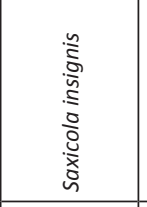 & 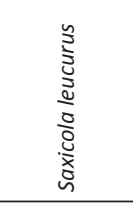 & 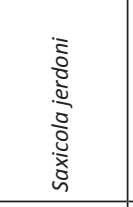 & 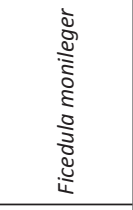 & 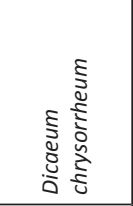 & 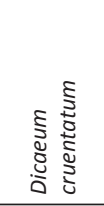 \\
\hline 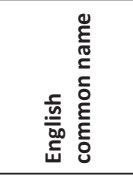 & 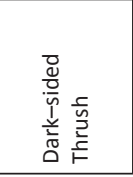 & 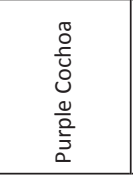 & 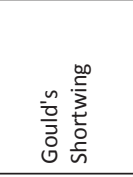 & 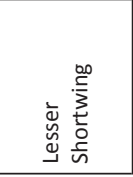 & 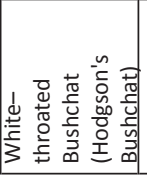 & 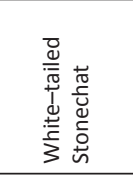 & 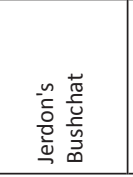 & 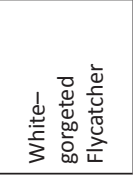 & 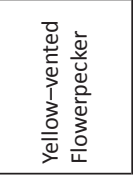 & 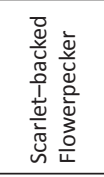 \\
\hline
\end{tabular}




\begin{tabular}{|c|c|c|c|c|c|c|}
\hline 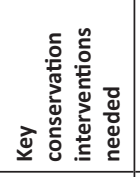 & 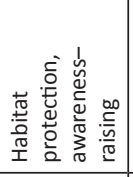 & 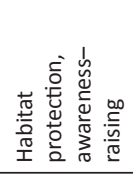 & 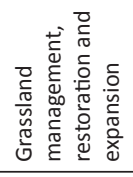 & 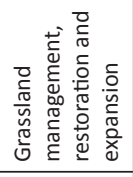 & 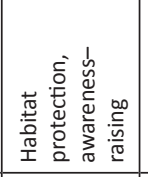 & 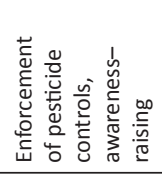 \\
\hline 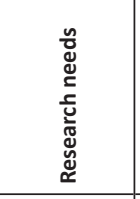 & 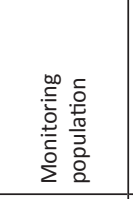 & 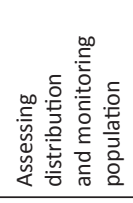 & 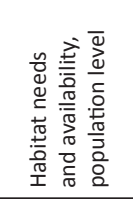 & 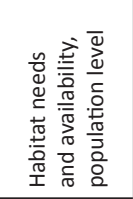 & 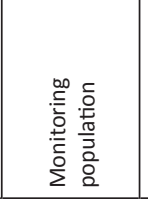 & 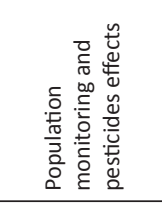 \\
\hline 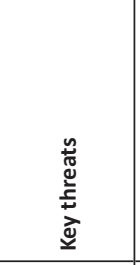 & 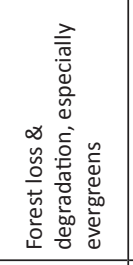 & 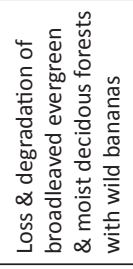 & 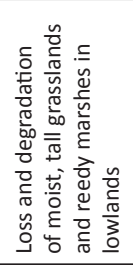 & 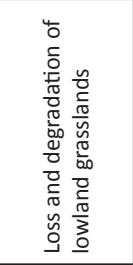 & 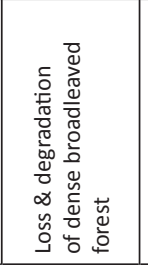 & 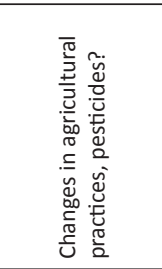 \\
\hline 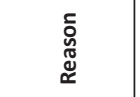 & $\sim$ & $\rightarrow$ & $\sim$ & \begin{tabular}{l}
0 \\
\multirow{\sigma}{*}{}
\end{tabular} & $\sim$ & $\sim$ \\
\hline 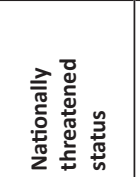 & 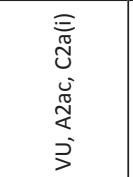 & 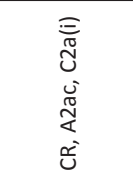 & 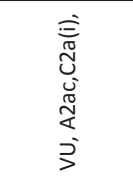 & 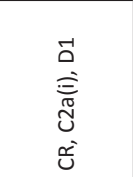 & 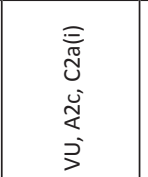 & $\begin{array}{l}\stackrel{y}{\tilde{\pi}} \\
\stackrel{x}{4} \\
\dot{u}\end{array}$ \\
\hline 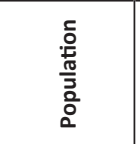 & & & & 啘 & & \\
\hline 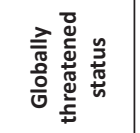 & & & & 3 & & 3 \\
\hline 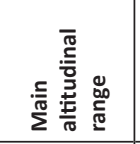 & 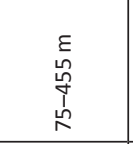 & $\begin{array}{l}\varepsilon \\
\stackrel{u}{0} \\
0 \\
\mu \\
N\end{array}$ & 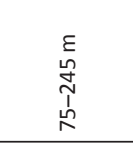 & $\begin{array}{l}\varepsilon \\
\varepsilon \\
0 \\
\stackrel{1}{1} \\
n \\
\\
\end{array}$ & 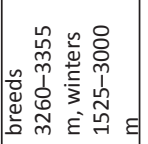 & $\begin{array}{l}\varepsilon \\
0 \\
0 \\
0 \\
1 \\
\\
\end{array}$ \\
\hline 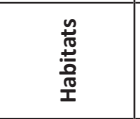 & 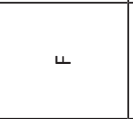 & 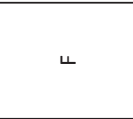 & ט & ৩ & 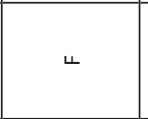 & ن \\
\hline 遻 & $\propto$ & $\propto$ & $\propto$ & $\propto$ & $\propto$ & $\xi$ \\
\hline 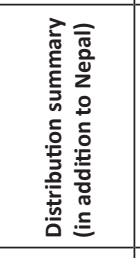 & 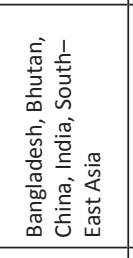 & 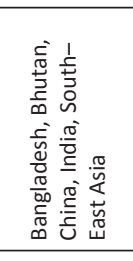 & 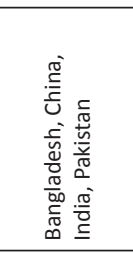 & 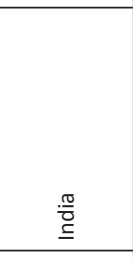 & 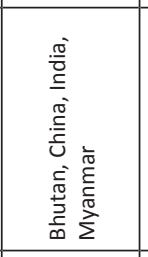 & 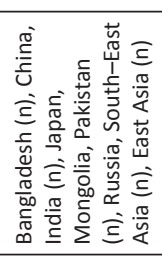 \\
\hline 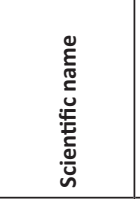 & 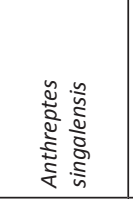 & 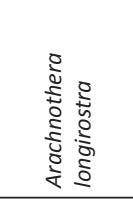 & 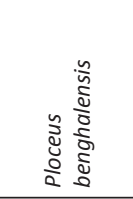 & 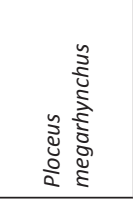 & 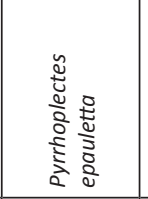 & 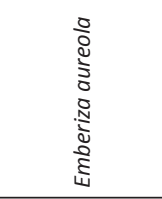 \\
\hline 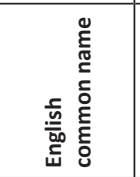 & 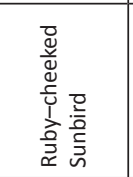 & 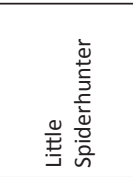 & 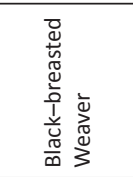 & 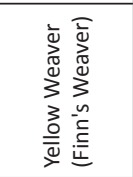 & 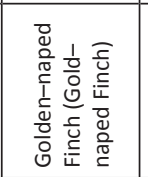 & 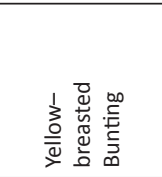 \\
\hline
\end{tabular}


Wildlife Reserve (Carol Inskipp pers. obs. December 2010). Within five years Mikania engulfed a large chunk of the reserve's marshes and terrestrial habitats at Koshi (Baral 2002a). Mikania is a climber that can very quickly cover trees and shrubs as well as the entire forest floor, thus making it impossible for bird species to feed on the ground. Terrestrial-feeding species, such as thrushes, pipits, as well as some babblers that require open forest floors, with or without decaying leaves, are all affected (Baral 2002a).

\section{Climate change}

The impacts of climate change on birds in Nepal are unknown, although are believed to be significant. Climate change is already having a significant impact on the Himalayan environment. Glacial melt is increasing as temperatures rise and will lead to increased summer flows in some river systems for a few decades, followed by a reduction in flows as the glaciers disappear (Baral 2002b). Ibisbill Ibidorhyncha struthersii, which breeds in braided river channels with shingle banks in glacial valleys, is likely to be directly threatened (Baral 2002b). A preliminary study of an Ibisbill breeding population in Kyanjin, upper Langtang in 2010 recorded 25 individuals, which included the very low number of only seven subadults. The researchers considered that the birds' poor breeding success resulted from landslides that have swept away an important portion of their habitat (Ghimire \& Thakuri 2010).

Some of Nepal's threatened birds are largely confined to protected areas, for example a number of grassland species. As the climate changes, habitats in these protected areas may eventually become unsuitable for these species. However, as natural habitats outside protected areas have been converted to agriculture or developed, these birds have nowhere to go.

Forktails, dippers, wagtails and river redstarts rely on invertebrate food supplies. It is possible that they could be indicators for understanding climate change impacts in the Himalaya because, as river flows are reduced, the birds' invertebrate prey will decline (Baral 2002b).

Many forest birds, including a high proportion of threatened forest species, depend on moist forests and are likely to be affected if the climate becomes drier.

\section{RESPONSES FOR NEPAL'S BIRDS - WHAT HAS BEEN DONE SO FAR}

\section{Conserving and protecting sites}

Nepal's protected area system: Nepal's protected area system (except buffer zones) covers over $20 \%$ of the country and includes 10 national parks, four reserves and six conservation areas. Since 2004 the government has established four new protected areas. Most protected areas have buffer zones which cover nearly $4 \%$ of the country's total land area.

A total of 16 of Nepal's 20 protected areas are managed by the Department of National Parks and Wildlife Conservation (DNPWC), Government of Nepal. Annapurna, Gaurishankar and Manaslu Conservation Areas are managed by the National Trust for Nature Conservation (NTNC). The NTNC was established in 1982 as a not-for-profit organisation, working in Nepal for nature conservation. Kanchenjunga Conservation Area is managed by a Council, the Kanchenjunga Conservation Area Management Council (KCAMC), which is a local body formulated from user groups and committees.

Limited or insufficient resources and capacity has impeded the ability of both the DNPWC and NTNC to adequately conserve protected areas and species.

While Nepal's protected area network is impressive in coverage, of Nepal's 27 IBAs only 13 are fully protected, with 12 unprotected and two partially protected. However, the Nepal Government's Fourth Report to the CBD in 2009 clearly stated that some management status would be given to three unprotected IBAs (Phulchoki Mountain Forest, Farmlands of Lumbini and Mai Valley).

Government legislation and policy: There are many government policies that support conservation efforts in Nepal, including the National Conservation Strategy for Nepal (National Planning Commission Secretariat, His Majesty's Government of Nepal 1988) that was endorsed as policy in 1988.

The recommendations of the Sustainable Development Agenda for Nepal (His Majesty's Government of Nepal, National Planning Commission and Ministry of Population and Environment 2003) build on proven, successful programmes at grassroots level. The national Community Forestry Programme (Ojha et al. 2009) demonstrates the high potential of participatory management as a means to promote sustainable development, for example.

The CBD has been enforced since February 1994. In 2002, a comprehensive Nepal Biodiversity Strategy was developed to fulfil Nepal's obligations to the CBD. This serves as an overall framework for the conservation and sustainable use of biodiversity and biological resources in the country. The Nepal Biodiversity Strategy Implementation Plan was developed in 2006; its overall goal is to achieve the NBS objectives during the period 2006-2010. 
The Ramsar Convention on Wetlands of International Importance especially as Waterfowl Habitat was ratified by Nepal in December 1987.

\section{Looking after habitats}

Community forests: In 1978, the Panchayat Forests and Panchayat Protected Forests regulations were introduced, enabling the Forest Department to return control and ownership of forests to local communities. The result was that, by April 2009, one-third of the population was participating in community managed forests, directly managing more than one-fourth of Nepal's forest area (Ojha et al. 2009). This approach has proved to be an effective way of conserving forests and biodiversity in many areas, especially where pressures on forests are high (Thapa 2007).

The National Wetland Policy and Ramsar: In 2003, the National Wetland Policy was agreed, with aims to conserve, manage and promote the wise and sustainable use of wetlands, particularly through collaboration of communities.

As part of its obligation under the Ramsar Convention, the Government has designated nine Ramsar Sites Wetlands of International Importance; a further six wetlands within IBAs could also qualify. The National Lake Conservation Development Committee was formed in 2006 with the objectives of conserving Nepal's lakes (Pokharel \& Shah 2006).

New guidelines for grassland management: New guidelines for grassland management-including requirements for birds-are being developed by DNPWC.

The banning of pesticides: Since April 2001, the Government has banned persistent chemical pesticides for use in agriculture and health. However, illegal import of pesticides remains a serious concern (Nepal Forum for Justice 2006). In addition, Nepal's National Agricultural Perspective Plan has emphasised the Integrated Pest Management (IPM) approach to try and reduce pesticide use, although very few individuals are IPM-trained (Atreya 2007).

\section{Saving species from extinction}

Protection by national law: Only nine species are protected by the National Parks and Wildlife Conservation Act - 2029 (1973). Recommendations for updating this list by the NGO Himalayan Nature are in the process of being adopted by the DNPWC.

Bird surveys: In recent years, several NGOs, including Bird Conservation Nepal (BCN), Bird Education Society (BES) and Biodiversity Conservation Society Nepal
(BIOCOS Nepal), and many individual Nepalis, have carried out bird surveys, chiefly of globally threatened species.

For instance, a survey of the population and distribution of White-throated Bushchat Saxicola insignis highlighted the importance of Sukla Phanta Wildlife Reserve as a major wintering area for the species (Baral 1998).

Mainly using Nepalese fieldworkers, the World Pheasant Association (WPA) has carried out pheasant population survey monitoring at Pipar in the Annapurna Conservation Area since 1979. Populations of all pheasant and partridge species at Pipar, including the nationally threatened and globally Near Threatened Satyr Tragopan Tragopan satyra have been regularly and frequently monitored (Anonymous 2010).

The waterbird monitoring programme which is coordinated by Wetlands International is the longest running annual bird monitoring programme in the country.

Bird Conservation Nepal's Vulture Conservation Programme: The Vulture Conservation Action Plan for Nepal (2009-2013) (Department of National Parks and Wildlife Conservation 2009) has helped prioritise and streamline vulture conservation activities. BCN and its partners are carrying out surveys of vulture populations and breeding success, as well as nationwide surveys of veterinary institutions to monitor the use of diclofenac. Wherever diclofenac is found, efforts are made to persuade the users to replace it by vulturesafe meloxicam. Samples of livers of animal carcasses available as food for vultures are being collected to test for diclofenac presence and so enable a better understanding of the threat to vultures from this drug in Nepal (Department of National Parks and Wildlife Conservation 2009).

Vulture Safe Zones are being set up by declaring areas free of diclofenac. As a first phase, 13 districts in Nepal are being targeted with a view to expanding the programme across Nepal and India. Advocacy campaigns include awareness work with the veterinary community, local community, district level decision makers, school children, and custom officials. (DNPWC/MoFSC/GoN 2009).

\section{Raising awareness}

Local communities: WPA has a strong education and conservation programme in Pipar, which is home to five out of six of Nepal's Himalayan pheasant species (Anonymous 2010), while BCN is building the capacity of grassroots conservation groups at IBAs. 
Since 2004, the Bird Education Society (BES) has been carrying out successful annual conservation awareness programmes for farmers who live in Chitwan National Park buffer zone. Many birds which inhabit the National Park also feed in the agricultural fields in the park's buffer zone, where they are at risk from pesticide use, notably the globally threatened Lesser Adjutant Leptoptilos javanicus. The BES programmes focus on providing farmers with the necessary knowledge and skills to practice effective micro-organism (EM) technology and learn about IPM (Subedi 2007).

Improving awareness more generally: A wide range of educational and conservation awareness activities are carried out by NGOs in Nepal. These include producing materials on birds and conservation, organising birdwatching for the general public, and running clubs for schools.

Another example is BCN's radio programme "Panchi Sansar" which conveys a bird conservation message to about $10 \%$ of Nepal's population every fortnight. The programme has proved a very effective communication tool, enabling $\mathrm{BCN}$ to reach the general public (BCN 2010).

An impressive campaign to raise awareness of the plight of owls has been carried out by Raju Acharya since 2008. At least four million people were informed about the campaign by local, regional and national newspapers, radio and television. A total of 166 owl conservation awareness camps in six districts had been carried out up to the end of 2010 (Acharya 2011).

\section{DISCUSSION}

The generosity of many more bird observers in Nepal in providing their unpublished records has led to the 2010 study being a more complete assessment of species' threat status than was possible previously.

Limitations of this study and the two previous assessments of Nepal's threatened birds are that not all species have been comprehensively assessed to identify those that might qualify for Near Threatened status. Passage migrants and vagrants were excluded because it was considered that the main threats to these species in particular may lie elsewhere. However, some passage migrants may use important stop-over sites where threats may be high, such as Koshi Tappu Wildlife Reserve. A new study is currently underway, as part of DNPWC's commitment to prepare animal red data lists under the auspices of the Zoological Society of London and National Trust for Nature Conservation, which is considering the threat status of all bird species recorded in Nepal to produce a National Red Data Book of Nepal's Birds. The work undertaken in this study will be used for this more comprehensive review.

Since 2004, more research work has been carried out to identify threats and the extent of their impact on Nepal's birds, and so we understand them better: for example, the impacts of diclofenac on White-rumped Vulture Gyps bengalensis and Slender-billed Vulture $G$. tenuirostris (Gautam \& Baral 2009, 2010); finding new vulture nesting sites, including in Arghakhanchi District where five vulture species breed (Bhusal 2010); research work on owls which has revealed the threats from trade (Acharya \& Ghimirey 2009); and a recent desk study which has shown the high impacts of agricultural changes (Inskipp \& Baral 2011).

Declines are not taking place as quickly as a simple comparison of the figures between the two assessments, in 2004 and 2010, indicates. More time was available for the 2010 study and, significantly, more records have been received from a larger number of contributors, revealing a truer picture of the state of Nepal's birds. With the improved knowledge resulting from the more detailed analysis carried out in 2010 it has been realised that in 2004 some species were more threatened than considered at that time, and should have been categorised as Critically Endangered or Endangered instead of Vulnerable; also that some species not picked up as Nationally Threatened in 2004, actually were.

Nevertheless a comparison between the results of the 2004 and 2010 assessments shows that the status of Nepal's birds has deteriorated. It is clear that some threats have certainly worsened, for example loss and degradation of forests, pressure on grasslands, the spread and intensification of agriculture and, most especially, the wide range of threats facing wetlands. However, positive responses have increased too, for instance the raising of conservation awareness, spread of community forestry and some projects benefiting local livelihoods.

Nepal is fortunate, compared with many developing countries, in that long-term and continuing interest in its bird life has provided a solid basis for recording and interpreting the changes that are taking place. Such documentation raises awareness of the importance of conservation and helps to focus on the highest priorities. As a result, there are more effective interventions than in places without such interest, for example targeted at species with the highest risk of global extinction (e.g. vultures) and at sites which are most important for biodiversity. That said, resources and capacity are still limiting factors, and many priorities remain that need to 
be addressed. The documentation has made possible the identification of some of the key actions needed (see Recommendations below). These should be high priorities for NGOs and government to consider as they revise their National Biodiversity Strategies and Action Plans (NBSAPs) in the light of the new CBD Targets for 2020.

\section{RECOMMENDATIONS FOR FUTURE NGO ACTION}

\section{Safeguarding sites}

1. Advocate protection of Important Bird Areas (IBAs), for example Dharan Forests, and potential IBAs like Khadara Phanta in lowland grasslands, and Khandbari-Num forests.

2. Lobby the Department of National Parks and Wildlife Conservation (DNPWC) to give management status to three unprotected IBAs as promised.

3. Promote suitable management for birds in protected IBAs, especially in lowland grasslands.

\section{Conserving habitats}

1. Lobby the Government to enforce existing pesticide regulations.

2. Encourage the use of Integrated pest management (IPM) and of effective micro-organism technology (EM).

3. Lobby the DNPWC to implement the new guidelines for grassland management.

\section{Saving species}

1. Lobby the DNPWC to ensure the adoption of updates to Nepal's protected bird list that have been provided by Himalayan Nature. In addition, lobby the DNPWC and provide expertise if necessary to ensure that the law protecting these species is enforced.

2. Conduct systematic surveys and undertake conservation activities for key threatened species, especially those that are declining: globally threatened species, e.g., Swamp Francolin Francolinus gularis, flagship species, e.g., Bengal Florican Houbaropsis bengalensis, and species that have not been recorded for at least 10 years, e.g., Silver-eared Mesia Leiothrix argentauris.

3. Carry out survey work to try and assess the impacts of climate change on bird species.

\section{Empowering people and raising awareness}

1. Carry out programmes to raise the awareness of local communities in IBAs where little or no similar work has been carried out previously, e.g., Dang Deukhuri Forests and West Rapti Wetlands, and in villages around Khaptad National Park.

2. Carry out training programmes for farmers in the use of EM technology and IPM in buffer zones of other protected areas, e.g., Bardia National Park and Sukla Phanta Wildlife Reserve.

3. Produce more radio programmes to raise conservation awareness, especially where such programmes have not been broadcast previously, e.g., in parts of the west central areas and the far western hills.

4. Start green clubs for schools in buffer zones of other protected areas and in unprotected IBAs, e.g., farmlands in Lumbini and the Tamur Valley and watershed.

\section{REFERENCES}

Acharya, R. (2011). Final report on owl conservation campaign (Tanahu, Dhading and Kaski districts). Submitted to World Owl Trust, UK \& Friends of Nature, Nepal, 13pp.

Acharya, R. \& Y. Ghimirey (2009). Final report on assessment of status, threats and the ethno-ornithological relationship and its extension for the conservation of owl in Nepal. Submitted to World Owl Trust, U.K, $56 \mathrm{pp}$.

Acharya, R., S. Thapa \& Y. Ghimirey (2006). Monitoring of the Cheer Pheasant Catreus wallichii in lower Kaligandaki valley, Mustang, Nepal. Report to King Mahendra Trust for Nature Conservation, Annapurna Conservation Area Project, Nepal, 17pp.

Anonymous (2010). Pheasants and schools at Pipar in the Annapurna Himalaya, Nepal <http://pheasant.org.uk/cons_scasia_pipar.aspx> Downloaded on 9 April 2011.

Atreya, K. (2007). Farmers' willingness to pay for community integrated pest management training in Nepal. Agriculture and Human Values 24: 399-409. <springerlink.com/content//38368m5I6r65814/> Downloaded on 9 April 2011.

Atreya, K. (2008). Health costs from short-term exposure to pesticides in Nepal. Social Science \& Medicine 67: 511-519.

Baral, H.S. (1998). Status, distribution and habitat preferences of Swamp Francolin Francolinus gularis in Nepal. Ibisbill 1: 35-70.

Baral, H.S. (2001). Community structure and habitat associations of lowland grassland birds in Nepal. PhD Thesis, University of Amsterdam, Amsterdam, 235pp.

Baral, H.S. (2002a). Invasive weed threatens protected area. Danphe 11(3): 10-11.

Baral, H.S. (2002b). Impact of climate change on Nepal's birds. Danphe 11(4): 6.

Baral, H.S. \& C. Inskipp (2004). The State of Nepal's Birds 2004. Department of National Parks and Wildlife Conservation, Bird Conservation Nepal \& IUCN Nepal, Kathmandu, 64pp.

Baral, H.S. \& C. Inskipp (2005). Important Bird Areas in Nepal: Key Sites for Conservation. Bird Conservation Nepal \& BirdLife International, Kathmandu and Cambridge, 242pp.

Baral, H.S., C. Inskipp, T.P. Inskipp \& U.R. Regmi (1996). Threatened Birds of Nepal. Bird Conservation Nepal \& Department of National Parks and Wildlife Conservation, Kathmandu, 13pp.

Bhusal, K.P. (2010). Survey of potential vulture nesting sites in northern belt of Arghakhanchi, Nepal. MSc Thesis. Tribhuvan University, Kathmandu.

Bird Conservation Nepal (2010). Annual Report 2009/10. Bird Conservation Nepal, Kathmandu, 34pp. 
Bird Conservation Nepal (2011). Bird Conservation Nepal (2011). Status of Nepal birds. <birdlifenepal.org/status of birds.htm> Accessed 8 April 2011.

Bird Conservation Nepal \& Department of National Parks and Wildlife Conservation (2011). The State of Nepal's Birds 2010. Bird Conservation Nepal \& Department of National Parks and Wildlife Conservation, Kathmandu, Nepal, 96pp.

Budhathapa, B.B. (2006). Status and distribution of Cheer Pheasant (Catreus wallichii) in Rara National Park. Report to World Pheasant Association \& Oriental Bird Club, 20pp.

Dahal, B.R. (2007). Effects of Water Hyacinth Eichhornia crassipes on aquatic birds at Koshi Tappu Wildlife Reserve, south-east Nepal. Danphe 16(1): 64-65. <birdlifenepal.org/publication.php> Downloaded on 10 April 2011

Department of National Parks and Wildlife Conservation (2009). Vulture Conservation Action Plan for Nepal(2009-2013). Government of Nepal, Ministry of Forests and Soil Conservation - Department of National Parks and Wildlife Conservation (DNPWC), Kathmandu, 25pp. <http://darwin.defra.gov.uk/documents/EIDPO022 /18126/ EIDPO022\%20AR2\%20App 8.\%20Vulture\%20Conservation\%20 Action\%20Plan\%20for\%20Nepal\%20Submitted\%20March\%20 2009.pdf> Downloaded on 28 October 2012

Forestry Nepal (2005). FAO releases final report of Forest Resources Assessment $2005<$ <orestrynepal.org/article/news/274> Downloaded on 9 April 2011.

Gautam, R. \& N. Baral (2009). Research and monitoring of Whiterumped Vulture (Gyps bengalensis) for seven years in Rampur, Nepal. Final report to The Peregrine Fund, USA, Royal Society for the Protection of Birds, UK \& Bird Conservation Nepal, 30pp.

Gautam, R. \& N. Baral (2010). Monitoring three endangered vulture species in the Pokhara valley, Nepal. Final report to the Royal Society for the Protection of Birds (RSPB) \& Bird Conservation Nepal, 12pp.

Ghimire, B.C. \& J. Thakuri (2010). Assessment of Ibisbill for the adaptation of climate change in central Nepal. Preliminary report submitted to National Adaptation of Action Project, Ministry of Environment, Nepal. 2pp.

Giri, T. (2002). Threats to birds at Koshi. Danphe 11(1): 35-36.

His Majesty's Government of Nepal, National Planning Commission and Ministry of Population and Environment (2003). Sustainable Development Agenda for Nepal, Kathmandu <http://www.npc.gov. $\mathrm{np} / \mathrm{new} /$ uploadedFiles/allFiles/Sustainable_Devlopment_Agenda_ Eng.pdf> Downloaded on 8 April 2010.

His Majesty's Government of Nepal and Ministry of Forests and Soi Conservation (2002). Nepal biodiversity strategy. His Majesty's Government of Nepal/Ministry of Forests and Soil Conservation, Kathmandu. <nepjol.info/index.php/AEJ/article/viewArticle/2114> Downloaded on 8 April 2010.

Inskipp, C. \& H.S. Baral (2011). Potential impacts of agriculture on Nepal birds. Our Nature (2010) 8: 270-312.

Inskipp, C. \& T. Inskipp (1991). A Guide to the Birds of Nepal-Second Edition. Christopher Helm, London. 400pp.

Inskipp, T. \& C. Inskipp (2010). A Bibliography of Nepal Birds. Unpublished, 64pp.

IUCN/SSC (2003). Guidelines for application of IUCN Red List criteria at regional levels. <http://cmsdata.iucn.org/downloads/2003_ guidelines_application_iucn_redlist_criteria_regional_levels.pdf $>$ Downloaded on 10 April 2009.

Kafle, G., M. Kafle, K. Balla \& B.K. Paudyal (2007). A review of threats to RAMSAR sites and associated biodiversity of Nepal. Tigerpaper 34(4): 1-5. <fao.org/asiapacific/rap/nre/links/tiger-paper/en/> Downloaded on 15 April 2011.

Kafle, G., M. Kafle, K. Balla \& B.K. Paudyal (2008). A review of threats to RAMSAR sites and associated biodiversity of Nepal (Part II). Tigerpaper 35(1): 9-11. <fao.org/asiapacific/rap/nre/links/tigerpaper/en/> Downloaded on 15 April 2011.

Ministry of Environment, Science \& Technology (MoEST) (2006). Rural Energy Policy. MoEST, Kathmandu. < http://www.moenv.gov. $\mathrm{np} /$ newwebsite/downloads/RE-Policy-2006.pdf > Downloaded on 28 October 2012.
National Planning Commission Secretariat, His Majesty's Government of Nepal (1988). Building on success: The National Conservation Strategy for Nepal. IUCN, Gland, Switzerland, 179pp. <http://pdf. usaid.gov/pdf_docs/PNABD226.pdf > Downloaded on 28 October 2012.

Nepal Forum for Justice (2006). Governmental and Public Awarenessraising on POPs. International POPs Elimination Project, Kathmandu, 8pp. <http://www.ipen.org/ipepweb1/library/ipep_ pdf reports/4nep\%20gov\%20and\%20public\%20awareness\%20 raising\%20on\%20pops.pdf> Downloaded on 28 October 2012.

Oaks, J.L., M. Gilbert, M.Z. Virani, R.T. Watson, C.U. Meteyer, B. Rideout, H.L. Shivaprasad, S. Ahmed, M.J.I. Chaudhry, M. Arshad, S. Mahmood, A. Ali K.K. \& Khan (2004). Diclofenac residues as the cause of vulture population decline in Pakistan. Nature 427: 630633. http://dx.doi.org/10.1038/nature02317.

Ojha, H., L. Persha \& A. Chhatre (2009). Community Forestry in Nepal: A Policy Innovation for Local Livelihoods and Food Security. Working Paper No. W09I-02. International Food Policy Research Institute, Ann Arbor, Michigan, 34pp. <http://www.ifpri.org/sites/default/ files/publications/ifpridp00913.pdf> Downloaded on 28 October 2012

Pokharel, S. \& K. Shah (2006). Lessons from Nepal on developing a strategic plan for ILBM - the case of Phewa Lake. <http://wldb. ilec.or.jp/ILBMTrainingMaterials/resources/nepal_strategy_ presentation.pdf> Downloaded on 12 April 2011.

Roberts, J., K.R. Tamang, S.R. Kumal, R.D. Mahato, N.Bdr. Gurau, A. Barlow, G. Malakar, C. McDougal \& M. Cotton (2002). Wetlands International Waterfowl Census January 2001, West Rapti and Narayani rivers. Danphe 11(1): 29-30.

Shakya, S. (1995). Bird massacre in Nepal. Bird Conservation Nepal Newsletter 4(3): 5.

Shultz, S., H.S. Baral, S. Charman, A. Cunningham, D. Das, G.R. Ghalsasi, M.S. Goudar, R.E. Green, A. Jones, P. Nighot, D.J. Pain \& V. Prakash (2004). Diclofenac poisoning is widespread in declining vulture populations across the Indian subcontinent. Proceedings of the Royal Society of London Series B-Biological Sciences 271: S458-S460. http://dx.doi.org/10.1098/rsbl.2004.0223.

Singh, P.B., L. Paudyel \& S. Sharma (2006). Survey of Cheer Pheasant Catreus wallichi in and around Dhorpatan Hunting Reserve, western Nepal. Report to World Pheasant Association, UK \& Oriental Bird Club, UK, 30pp.

Siwakoti, M. (2007). Mikania weed: a challenge for conservationists. Our Nature 5: 70-74. <nepjol.info/index.php/ON/article/ viewFile/801/770> Downloaded on 8 April 2011.

South Asia Co-operative Environment Programme (2010). Nepal. <sacep.org/default.htm> Downloaded on 9 April 2011.

Subedi, H. (2007). A bird conservation awareness camp for local farmers. Danphe 16(1): 43-44. <birdlifenepal.org/publication.php> Downloaded on 10 April 2011.

Subedi, T.R. (2008). Status and conservation of Red Panda Ailurus fulgens in Dhorpatan Hunting Reserve, Nepal. Rufford Small Grants for Nature Conservation Project. <ruffordsmallgrants.org/rsg/ projects/tulsi_ram_subedi> Downloaded on 9 April 2011.

Thapa, I. (2007). Community forestry: ideal for participatory biodiversity conservation. Danphe 16(1): 18-20. <http://www.birdlifenepal.org/ publication.php> Downloaded on 8 April 2010.

Thapa, Y.V. (2006). Constraints and approach for improving fertilizer supply for meeting domestic demand. Economic Policy Network Policy Paper 30. Report to Economic Policy Network \& Asian Development Bank, Kathmandu. <mof.gov.np/economic_policy/ pdf/Constraints_Approach.pdf> Downloaded on 9 April 2011.

Tyabji, H. (2002). The crisis of the rivers and streams in Royal Chitwan National Park. Danphe 11(1): 30-31. 
Author Details: CAROL INSKIPP has written a number of books and papers on the conservation, status, distribution and identification of Nepal's birds since 1985, mainly with her husband Tim and Hem Sagar Baral.

Hem SAgar BARAL has a PhD in Ecology of Nepal's Grassland Birds from the University of Amsterdam, The Netherlands and has worked on bird conservation for 25 years. He has held important positions in Bird Conservation Nepal, founded the non-governmental organisations Himalayan Nature and Nepalese Ornithological Union, and Nepal's only bird observatory. He has authored and coauthored several books and many papers on Nepal bird conservation

TIM INSKIPP has studied the distribution, status and identification of birds in Nepal since his first visit to the country in 1970 and has co-authored a number of books and papers on the subject.

ALISON STATTERSFIELD is Head of Science at Birdlife International, responsible for ensuring that the conservation programmes of BirdLife's Partner organisations, operating in over 100 countries, are based on global priorities derived from sound science.

Author Contribution: $\mathrm{Cl}$ drafted the text for this paper. For the project she assisted in: compiling and analysing species information and making the overall analysis of threats to Nepal birds, responses made and recommendations for the future. HSB assisted in compiling species information, coordinated field-based persons on recent updates, and helped to make the overall analysis of Nepal's bird conservation scenario. $\mathrm{Tl}$ assisted in the compilation of the bibliography on birds of Nepal and in compiling species information for this project. AS's contributions to the paper and project include guidance on methods, interpretation of results and contributions to drafting. 